\title{
PIPE SIZING:
}

FOR

SOLENOID/VLPC

CRYOGENIC SYSTEMS

D-ZERO ENGINEERING NOTE \# 3823.115- EN-416

February 20, 1995

rev. 6/26/95: changes in italics

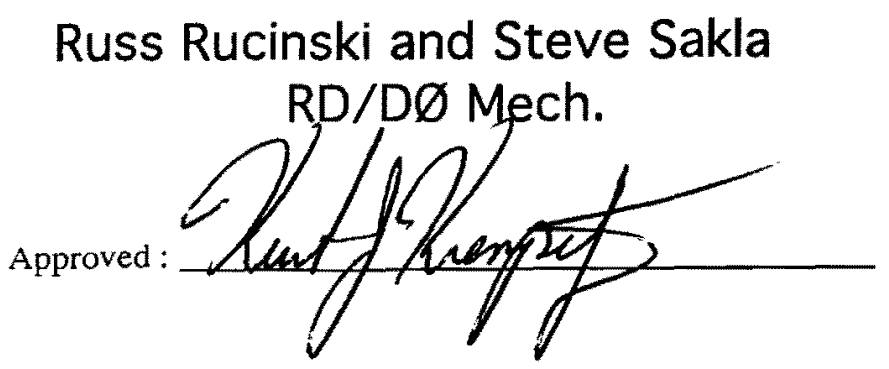




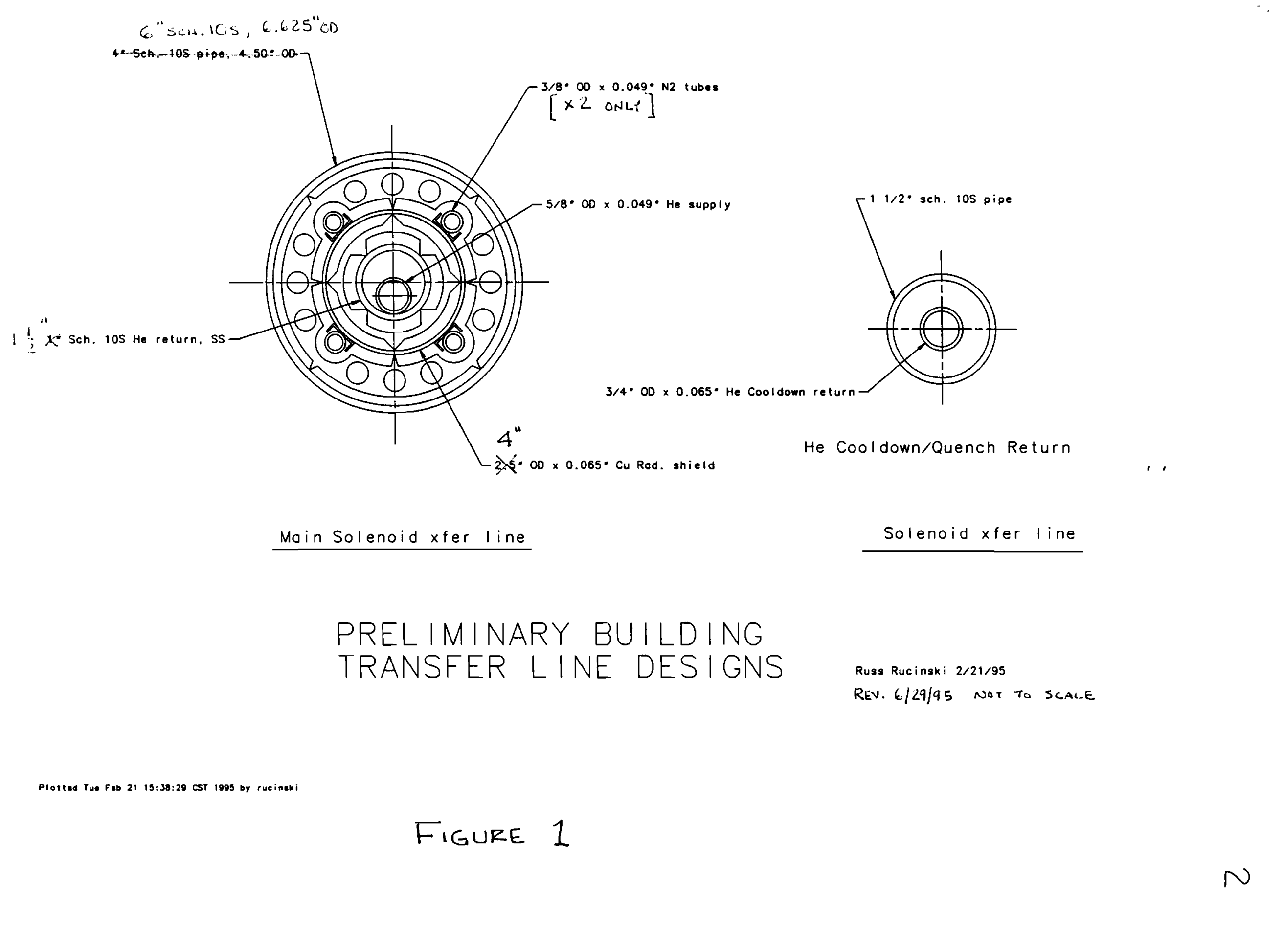


$\frac{1}{2}^{n}$

$3 \times 8^{\cdot} \cdot 00 \times 0.049^{\cdot}$ LN2 supply

$4 " 2500 \times 0.065 \cdot$ Cu Rad. shis.
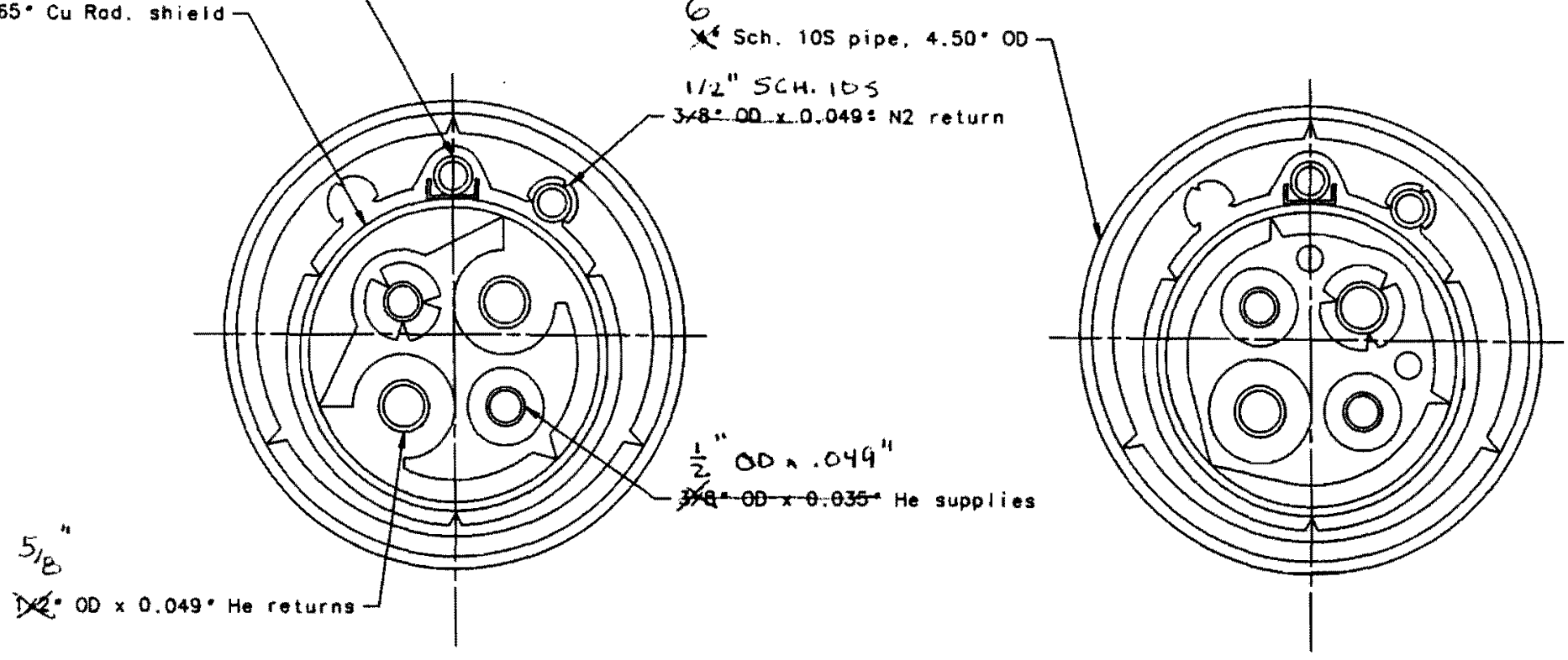

VLPC transfer line

PREL IMINARY BUILDING

TRANSFER LINE DESIGNS

Russ Rucinski 2/21/95

REV. 6/24/95 NUT TO SCALE

Figure 2 
Table 1 : solenoid Ppesections

\begin{tabular}{|c|c|c|c|c|c|c|c|c|c|c|c|c|}
\hline LINE : & ELUID & DESCFIPTON & TYPE & moM & 10 & $\begin{array}{c}\text { EIPEOR TUBE } \\
\text { SLE }\end{array}$ & $\begin{array}{l}\text { MSIDE } \\
\text { DIA. IIN.) }\end{array}$ & $\begin{array}{c}\text { LMEAB } \\
\text { LENGTH (FT) }\end{array}$ & $\begin{array}{c}\angle \text { OOF } \\
\text { ELBOWS }\end{array}$ & $\begin{array}{c}\text { ITEES } \\
\text { IHBURUN }\end{array}$ & $\begin{array}{c}\text { TEES } \\
\text { IHBU } \\
\text { RRANCH }\end{array}$ & VALYES \\
\hline 4 & LHE & SUPPLY (HUT3) & U-TUBE & LHE DEWAR & X-FER LINE & $3 / 4^{4}$ OD X .035 & 0.68 & $s$ & 2 & 0 & 0 & 0 \\
\hline 5 & LHE & SUPPLY & X.FER LNE & U-TUBE & U-TUBE & $518^{\prime \prime}$ OD X.049 & 0.527 & 125 & 9 & 4 & 0 & I DIVRTR. \\
\hline 6 & LHE & SUPPLY (HUT4) & U.TUBE & X-FER LINE & CONTROL DEWAR & $3 / 4^{\prime \prime}$ OD X .035 & 0.68 & 10 & 2 & 0 & 0 & 0 \\
\hline 7 & LHE & RETURN (HUTS) & U.TUBE & CONTROL DEWAR & X.FER LINE & $3 / 4^{\prime \prime} \mathrm{OD} \times .035$ & 0.68 & 10 & 2 & 0 & 0 & 0 \\
\hline 8 & LHE & RETURN & X.FER LNE & U-TUBE & U.TUBE & $\underset{\text { Concentric }}{\text { Duyo }}=$ & $\begin{array}{l}1.0 \\
-0.427\end{array}$ & 125 & 9 & 4 & 0 & $1 \mathrm{PV}, 1 \mathrm{CV}$ \\
\hline 9 & HE & RETURN & U-TUBE & X.FER LINE & X-FER LINE (NLPC) & $3 / 4^{\prime \prime}$ oD $\times .035$ & 0.68 & 3 & 2 & 0 & 0 & 0 \\
\hline 10 & LN2 & SUPPLY & U-TUBE & LIN HEADER & X-FER LINE & $\begin{array}{r}3 / 800 \times .049 \\
+200 \times 2035 \\
\end{array}$ & $\begin{array}{r}.277 \\
0.43 \\
\end{array}$ & 3 & 2 & 0 & 0 & 0 \\
\hline 11 & LN2 & SUPPLY & $X$ XEER LNE & U.TUBE & IEx & $\begin{array}{r}3800 \times .049 \\
\quad 00 \times .049 \\
\end{array}$ & $\begin{array}{l}271 \\
0402 \\
0\end{array}$ & 1 & 1 & 0 & 0 & 0 \\
\hline 12 & LN2 & SUPPLY (SHELLD) & X.FER LINE & TEE & U. TUBE & $3 / 8^{\prime \prime}$ on X.049 & 0.277 & 124 & 9 & 2 & 1 & $1 \mathrm{PV}$ \\
\hline 13 & $\mathrm{LN2}$ & SUPPLY (SHELD) & U.TUBE & X-FER LINE & CONTROL DEWAR & $1 / 2^{\prime \prime}$ OD $\times .035$ & 0.43 & 10 & 2 & 0 & 0 & 0 \\
\hline 14 & LN2 & $\begin{array}{l}\text { SUPPLY } \\
\text { (INTERCEPT) }\end{array}$ & X-FER LINE & TEE & U-TUBE & $3 / 8^{\prime \prime}$ OD X.049 & 0.277 & 124 & 9 & 2 & 1 & $1 \mathrm{PV}$ \\
\hline 15 & $\mathrm{LN}_{2}$ & $\mid \begin{array}{l}\text { SUUPLY } \\
\text { (INTERCEPT) }\end{array}$ & U-TUBE & X.FER LINE & CONTROL DEWAR & $1 / 2^{\prime \prime}$ OD $\times .035$ & 0.43 & 10 & 2 & 0 & 0 & 0 \\
\hline 16 & $\mathrm{LN}_{2}$ & RETURN (SHIELD) & U.TUBE & CONTROL DEWAR & X.FER LINE & $1 / 2^{\prime \prime}$ OD $\times .035$ & 0.43 & 10 & 2 & 0 & 0 & 0 \\
\hline 17 & $\mathrm{LN} 2$ & RETURN (SHELD) & X.FER LNEE & U.TUBE & U-TUBE & 318 " OD X.049 & 0.277 & 125 & 9 & 2 & 0 & 0 \\
\hline 18 & $\mathrm{LN}_{2}$ & RETURN (SHELL) & U-TUBE & $X$ FER LINE & TEE & $1 / 2^{\prime \prime}$ OD $\times .035$ & 0.43 & 1 & 1 & 0 & 1 & 0 \\
\hline 19 & LN2 & $\begin{array}{l}\text { RETURN } \\
\text { (NNTERCEPT) }\end{array}$ & U.TUBE & CONTROL DEWAR & $X$-FER LINE & $1 / 2^{\prime \prime}$ OD $\times .035$ & 0.43 & 11' & 2 & 0 & 0 & $(3$ \\
\hline 20 & $1 . \mathrm{N} 2$ & \begin{tabular}{|l} 
RETURN \\
(INTERTEPT)
\end{tabular} & X-FT:R IIN: & 1. TU1B: & | (1. TIIISB: & 18 OD X & 0.277 & 125 & 2 & 2 & in & $a$ \\
\hline 22 & $\mathrm{LN} 2$ & $\begin{array}{l}\text { RETURN } \\
\text { (INTERCEPT) }\end{array}$ & U.TUBE & $X$-FER LINE & TEE & $1 / 2^{\prime \prime}$ OD $\times .035$ & 0.43 & 1 & 1 & 0 & 1 & 0 \\
\hline 23 & LN2 & RETURN & U-TUBE & TEE & GNR VENT HEADER & $1 / 2^{\prime \prime}$ on $\times .035$ & 0.43 & 5 & 1 & 0 & 0 & 0 \\
\hline 24 & $\mathrm{HE}$ & $\begin{array}{l}\text { (COOLDOWN } \\
\text { QUENCH) }\end{array}$ & U.TUBE & CONIROL DEWAR & X-FER LINE & $\begin{array}{r}1 / 204.10 \\
-3,400-035\end{array}$ & $\begin{array}{c}6.74 \\
0.58 \\
\end{array}$ & 10 & 2 & 0 & 0 & 0 \\
\hline 25 & HE & $\begin{array}{l}\text { (COOLDOWN/ } \\
\text { QUENCH) }\end{array}$ & X-FER LNE & U.TUBE & U.TUBE & 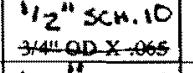 & $\begin{array}{l}.674 \\
.0 .62\end{array}$ & 125 & 9 & 2 & 0 & 0 \\
\hline 26 & HE & $\begin{array}{l}\text { (COOLDOWN' } \\
\text { QUENCH) }\end{array}$ & U-TUBE & X-FER LINE & $\begin{array}{l}\text { HE OOOLDOWN } \\
\text { RETURN }\end{array}$ & $\begin{array}{c}1 / 2 \sin .10 \\
-1 / 400-x-035\end{array} \mid$ & $\begin{array}{r}6.74 \\
0.68 \\
\end{array}$ & 5 & 2 & 0 & 0 & 0 \\
\hline 27 & HE & $\begin{array}{l}\text { SUPPLY } \\
\text { COOOLDOWM }\end{array}$ & U-TUBE & COOLDOWNLINE & X-FER LINE & $3 / 4^{\prime \prime}$ on $\times .035$ & 0.68 & $s$ & 4 & 0 & 0 & DIVRT \\
\hline
\end{tabular}


TABLE 2: vPcoppes sectows

\begin{tabular}{|c|c|c|c|c|c|c|c|c|c|c|c|c|}
\hline LINE : & ELUI? & DEscaiption & TreE & EROM & ro & \begin{tabular}{|c|} 
PIPE OR TUBE \\
SIZE \\
\end{tabular} & INASPE & $\frac{\text { LK. LENGTH }}{\text { IFTI }}$ & $\begin{array}{c}\text { LOF } \\
\text { EIBOWE }\end{array}$ & $\begin{array}{c}\text { DTEES } \\
\text { THAURUA }\end{array}$ & $\begin{array}{l}\text { LTEES } \\
\text { IHRUN } \\
\text { BRANCH }\end{array}$ & YALYES \\
\hline 38 & IE & Suepry & UTRE & Himx6 & AMFEEDCAN & $1 / 2=00 \times .049$ & $\begin{array}{l}0.402 \\
\end{array}$ & 5 & 2 & 0 & 0 & 0 \\
\hline 30 & LWE & suppry & ULTUEE & LHE DEWAR & AHFEEDCAN & $1 / 2^{n}$ OD X .049 & 9.402 & 5 & 2 & 0 & 0 & $a$ \\
\hline 40 & IE & WEST HE SUPPLY & $X$ XFR UNE & UTuaE & 3-WAYVALVE & $1 / 2^{1600 \times 0} \times 04$ & .402 & 2 & 1 & 1 & 0 & 0 \\
\hline 4 & HE & 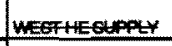 & $x$ EnE & Uanes & OWHAYvate & & & $z$ & \pm & + & $\theta$ & $\theta$ \\
\hline 42 & IE & WEST HE SUPPLY & XFER UNE & 3-WAY VALVE & UTues & 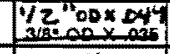 & .402 & 123 & 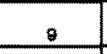 & 3 & 0 & $10 \mathrm{~V}$ \\
\hline 43 & IE & WEST HESLPPLYY & LLTueE & CHFEEDCAN & BAYONET CAN & - & 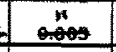 & 12 & 2 & 0 & 0 & 0 \\
\hline 44 & IE & WEST HE SUPPLY & $X+$ ER LNE & BAYONET CAN & VALVE BOX & \begin{tabular}{|c|}
$x$ \\
$x+\infty$
\end{tabular} & * & 47 & 6 & 1 & 0 & 0 \\
\hline 45 & EE & WEST HE SUPPLY & XFER UNE & VALVE BOX & MLC & 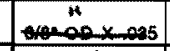 & $00+$ & 24 & 2 & 0 & 0 & 0 \\
\hline 46 & HE & EASTHE SUPPLY & $X$ XER UNE & UTUBE & 3-war vaLVE & 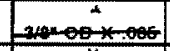 & 告 & 2 & 0 & 1 & 1 & $\circ$ \\
\hline 47 & IE & EAST HE SUPPLY & $X$-FER UNE & UTUEE & 3-WAY VALVE & -11 & "11 & 2 & 0 & 0 & 1 & 0 \\
\hline 48 & He & EAST HESUPPLY & $X$ FER LNE & 3-WAY VALVE & LUREE & - & ئم & 123 & 9 & 3 & 0 & $10 \mathrm{v}$ \\
\hline 49 & HE & EAST HE SUPPLY & UTUEE & CHFEEDCAN & BAYONET CAN & $-00 \times 1005$ & \begin{tabular}{|c|}
1 \\
\end{tabular} & 12 & 2 & 0 & 0 & 0 \\
\hline 50 & HE & EAST HE SUPPIY & X+FER UNE & BAYONET CAN & VALVE BOX & - & $=0.305$ & 47 & 6 & 1 & 0 & 0 \\
\hline 51 & HE & EASTHE SUPAYY & X-FER UNE & VALVEBOX & upe & $-000 \times 005$ & $\ddot{11}$ & 24 & 2 & 0 & 0 & 0 \\
\hline 52 & HE & EAST HE RETURAN & $X$ FER UNE & vore & VALVE BOX & -00 & 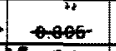 & 24 & $\hat{2}$ & 0 & 0 & IPV \\
\hline 53 & IE & EAST HE RETURN & XFER UNE & VALVE BOX & BAYONET CAN & $513^{10} 00^{*} \times 64$ & 49.527 & 47 & 6 & 0 & 0 & 0 \\
\hline 54 & EE & EAST HEREMURN & UTuRE & BAYONET CAN & COHFEEOCAN & $316^{*}$ oq $\times .035$ & 0.305 & 12 & 2 & 0 & 0 & 0 \\
\hline 55 & DE & EASTIEERETLRN & XfER UnE & CMFEEDCAN & ZWAY VALVE & $3 / 8^{*}$ of $\times .035$ & 0.105 & 123 & 8 & 3 & 1 & $10 \mathrm{~V}$ \\
\hline 56 & IE & EAST HE RETURN & $X$ XER UNE & 3.WAY VALVE & $\begin{array}{l}\text { WJ LNE ¿COOLDOWN } \\
\text { RETUPON }\end{array}$ & $380.00 \times .035$ & $0 . \$ 05$ & 2 & 2 & 1 & \pm & $1 \mathrm{CV}$ \\
\hline 57 & IE & EAST HE RETURN & $x+$ ER UNE & 3-WAY VALVE & VJUNE (NALVE BOX) & $3 / 8^{*} 00 \times .035$ & 0,005 & 2 & 2 & 2 & 1 & $\mathrm{cov}$ \\
\hline 58 & HE & WEST HERERURN & XFER UNE & npe & VALve BOX & $318 * \phi \times .035$ & 0,305 & 24 & 1 & 0 & 0 & IPV \\
\hline 50 & HE & WESTHERETURN & XFR UNE & VALVE BOX & BAYONET CAN & $3 / 8.90 \times .035$ & 0,305 & 47 & 5 & 0 & 0 & 0 \\
\hline so & IE & WESTHE RETURN & utueE & BAYONET CAN & CHFEEDCAN & $3 / 8.0 \times .035$ & 0305 & 12 & 2 & 0 & 0 & 0 \\
\hline 81 & HE & WEST HE RETURN & $X$ FER UNE & CHFEDCAN & 3.WAY VALVE & $3 / 8^{*}$ do $\times .035$ & 01305 & 123 & $\theta$ & 2 & 0 & $10 \mathrm{~V}$ \\
\hline 62 & WE & WEST HEAETURN & XFER UNE & 3-WAY VALVE & $\begin{array}{l}\text { WU UNE (COOLOOWN } \\
\text { RETUREN }\end{array}$ & $3 / 8 \cdot$ op $\times .035$ & opos & 2 & 2 & 0 & 2 & $1 \mathrm{cV}$ \\
\hline 63 & IE & WEST HE RETLRN & $X$ XFER UNE & 3-WAY VALVE & WJ LINE NVALVE BOXI & $3 / 8 \cdot 0 \times \times .035$ & ollos & 1 & 1 & 0 & 2 & $1 \mathrm{cV}$ \\
\hline 64 & IE & WEST HERETRN & XFER UNE & 3-WAY VALVE & TEE & 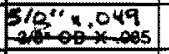 & $\begin{array}{r}.527 \\
0.06\end{array}$ & 2 & 1 & 1 & 2 & $1 \mathrm{cV}$ \\
\hline 65 & HE & RETLPN & XfER UNE & $\begin{array}{l}\text { UTUBE FROM SOL X- } \\
\text { FERLE }\end{array}$ & TE & $\begin{array}{l}7 \\
1 / 2^{\prime \prime} \text { OD X .049 } \\
\end{array}$ & 0.402 & 1 & 1 & 2 & 1 & 0 \\
\hline 80 & $E$ & RERTR & LTUBE & AHFEED CAN & $\begin{array}{l}\text { OOOLDOWN RETURN } \\
\text { UNE }\end{array}$ & $\begin{array}{r}3 / 400 \times .05 \\
-00 \times 049 \\
\end{array}$ & $\begin{array}{r}.68 \\
-002 \\
\end{array}$ & 5 & 2 & 0 & 0 & 0 \\
\hline 67 & HE & Irenran & UTHBE & AM FEED CAN & VALVE BOX & $344^{*} .00 \times .035$ & 0.68 & 4 & 0 & 0 & 0 & 0 \\
\hline 68 & LN2 & suppry & UTRE & LN HEADER & AHFEED CAN & $1 / 2^{2} 08 \times .049$ & $\begin{array}{l}.402 \\
.929 \\
\end{array}$ & 3 & 2 & 0 & 0 & 0 \\
\hline 60 & LN2 & SUPPLY & XFER UNE & AHFEEDCAN & CHFEEO CAN & $\mid 12^{10} \Delta x, 019$ & $\begin{array}{r}402 \\
0.272 \\
\end{array}$ & 125 & 9 & 2 & 0 & 0 \\
\hline 70 & LNZ & supper & UneE & CMFEEDCAN & BAYONET CAN & 34000 & 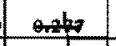 & 12 & 2 & 0 & 0 & 0 \\
\hline 71 & LN2 & supper & XFERUNE & BAYONET CAN & VALVE BOX & $\begin{array}{r}-049 \\
\end{array}$ & $a+2$ & 47 & $\underline{B}$ & 1 & 0 & 0 \\
\hline 72 & LN2 & supper & $x+$ FR UNE & VALVEBOX & VURCWEST & $3 / 8^{\prime \prime}$ on $\times .049$ & 0.277 & 24 & 3 & 1 & 1 & IPV \\
\hline 73 & LN2 & Suppey & XFERURE & VALVEBOX & VIPCEAST & $3 / 8^{\prime \prime}$ on $\times .049$ & 0.277 & 24 & 3 & 1 & 1 & IPV \\
\hline 74 & LN2 & supply & $X$ XER UNE & VLPC WEST & VALVE BOX & $3 / 8^{\prime \prime} d f \times .049$ & 0.37 & 24 & 0 & 0 & 1 & 0 \\
\hline 75 & LN2 & Suppir & $X$ FR UNE & YLPCEAST & valve BOX & $\begin{array}{r}1 / 2 \\
3 / 8\end{array}$ & $\begin{array}{r}402 \\
0.27\end{array}$ & 24 & 1 & 1 & 0 & 0 \\
\hline 76 & W2 & RETUNN & $x$ fRR UNE & VALVE BOX & BAYONET CAN & 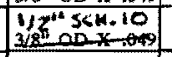 & .674 & 47 & 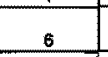 & 1 & 0 & $\circ$ \\
\hline 77 & LN2 & PETLPN & UTuBE & BAYONET CAN & CHFEED CAN & 3/8" of $x$.049 & 0,247 & 12 & 2 & 은 & 0 & 0 \\
\hline 78 & LWR & RERURN & XFER LME & CHFEEDCAN & ANFEED CAN & $3 / 8^{n} d / x, 049$ & $a+5$ & 125 & 9 & 2 & 0 & 0 \\
\hline 70 & LN2 & RETURN & ULTUBE & AHFEED CAN & LON VENT HEADER & 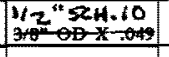 & .671 & 5 & 2 & 0 & 0 & 0 \\
\hline & * VLPCSE & ERPENTINELENGTH & $=16 \mathrm{FT}$ & & & & & & & & & \\
\hline & * $\angle O F$ EL & BOWS IN SERPEN & $N E=18$ & & & & & & & & & \\
\hline
\end{tabular}




\section{Solenoid Helium Steady State Pressure drops}

Some preliminary calculations were done to estimate the steady state helium heat loads, flowrates and pressure drops expected for each section of piping. See appendices $\mathrm{I}, \mathrm{J}$, and $\mathrm{K}$. The total pressure drop from the tubing sizes were on the order of $3.2 \mathrm{psi}$ as shown in the summary table in appendix K, page K12. Essentially all this pressure drop occurred in the long return pipe, line \# 8 . The pressure drop was based on line \# 8 being a concentric tube with hydraulic radius of $0.347 "$. The return pipe, line $\# 8$ was looked into further. See appendix $L$. It was determined that a $11 / 2^{\prime \prime}$ sch. 10 pipe will be used giving a hydraulic radius of 1.057". The total steady state pressure drop then is on the order of 0.1 psi for the piping. Allowing all the pressure drops to occur across control valves.

\section{Solenoid Nitrogen flowrates and pressure drops}

The nitrogen flowrate requirements for cooldown and steady state are considered in calculations in appendix F. The maximum pressure drop allowed for the nitrogen circuit inlet to outlet is $30 \mathrm{psi}$, based on the current storage dewar operating pressure. Per the Solenoid bid spec., the required nitrogen cooldown flowrate shall not exceed $5 \mathrm{~g} / \mathrm{s}$. With the current selected pipe sizes nitrogen flow will less be than $5 \mathrm{~g} / \mathrm{s}$ until the return piping fluid is less than $150 \mathrm{~K}$. This means initially we may cannot deliver the full $5 \mathrm{~g} / \mathrm{s}$. I feel this is acceptable however. The pressure drop for steady state nitrogen requirements is expected to be less than 1 psi (with control valves full open). See appendix G.

\section{VLPC Helium Cooldown flowrates}

The LHe line sizes for the VLPC were determined by first calculating the required flow rates during cooldown. These calculations are shown in "VLPC Cooldown \& Flow Rates Etc." of appendix D and are summarized in Table 7. A cooldown rate of $5 \mathrm{~K} / \mathrm{hr}$ was used in these calculations.

Table 7: VLPC COOLDOWN FLOW RATES

\begin{tabular}{|c|c|c|c|c|c|}
\hline STEP & $\begin{array}{c}\text { VLPC } \\
\text { TEMP } \\
\text { [K] }\end{array}$ & $\begin{array}{c}\text { GAS } \\
\text { TEMP } \\
\text { [N [K] }\end{array}$ & $\begin{array}{c}\text { GAS } \\
\text { TEMP } \\
\text { OUT [K] }\end{array}$ & $\begin{array}{c}\text { TIME } \\
\text { [hrs] }\end{array}$ & $\begin{array}{c}\text { MASS FLOW } \\
\text { RATE REQ'D } \\
\text { [g/s] }\end{array}$ \\
\hline 1 & 300 to 250 & 200 & 250 & 10 & .84 \\
\hline 2 & 250 to 200 & 150 & 200 & 10 & .89 \\
\hline 3 & 200 to 150 & 100 & 150 & 10 & .92 \\
\hline 4 & 100 to 90 & 80 & 90 & 4 & 3.1 \\
\hline
\end{tabular}


It was concluded that step 4's pressure drop would be about 3.6 times larger than step 1's. Therefore the cooldown lines were sized for a flow rate of $3.1 \mathrm{~g} / \mathrm{s}$ with an inlet temperature of $80 \mathrm{~K}$ and an outlet temperature of $90 \mathrm{~K}$ (refer to appendix D).

\section{VLPC Helium Cooldown Pressure drops}

A list of the various piping sections along with their corresponding lengths and number of elbows, tees, and valves. Each pipe section was given a number. See table 4 for a listing of the VLPC pipe sections.

Pressure drops due to friction, valves, tees, bends, etc. were then calculated for the given pipe sizes and inlet/exit states. The VLPC cooldown piping was first sized by assuming a pressure drop of $95 \mathrm{psig}$, an inlet temperature of $80 \mathrm{~K}$ GHe and an outlet temperature of $90 \mathrm{~K}$ through the supply and return lines respectively. Resistance coefficients were calculated for four segments of the piping. In the second step of the calculation, pressure drops were calculated for each segment taking into account the change in density due to changes in pressure. Summing the individual pressure drops resulted in a rough estimated pressure drop of $59.1 \mathrm{psi}$. These numbers are summarized in table 9 for the initial supply size of $3 / 8^{\prime \prime}$ OD and return size of 1/2" OD tubes. See Appendix $\mathrm{E}$ for the raw calculations. * After looking at steady state pressure drops, the line sizes for the supply and return were increased to $1 / 2^{\prime \prime} O D$ and 5/8" OD respectively. Therefore pressure drops listed in Table 9 will be less.

Table 9: VLPC Helium Cooldown piping pressure drop summary for $3 / 8$ " supply \& $1 / 2$ " return sizes. *

\begin{tabular}{|c|c|}
\hline PIPE SECTION & PRESSURE DROP (PSIA) \\
\hline Supply line 38 & 0.44 \\
\hline West supply lines + VLPC cryostat & 27.4 \\
\hline West return lines & 28.1 \\
\hline Return line 66 & 3.2 \\
\hline & \\
\hline & \\
\hline & $\mathbf{5 9 . 1}$ psi \\
\hline
\end{tabular}




\section{VLPC Helium Steady State Pressure drops}

The expected pressure drop for $3 / 8^{\prime \prime}$ OD $x 0.035$ wall supply tubing for a steady state flow of $5 \mathrm{~g} / \mathrm{s}$ and a transfer line heat load of $10 \mathrm{~W}$ was conservatively estimated at 4.6 psi per $100 \mathrm{ft}$. This prompted the increase in size to $1 / 2^{\prime \prime}$ OD $\times 0.049$ wall tubing. The calculated pressure drop for the two phase supply would be $1.2 \mathrm{psi}$ per $100 \mathrm{ft}$. Backing off on the conservatism and figuring approx. $200 \mathrm{ft}$. of equivalent length a real estimate of the supply pressure drop from the refrigerator to the VLPC cryostat is about 1 psi. See Appendix H, page H4.

The expected pressure drop for the return $5 / 8$ " OD $x 0.049^{\prime \prime}$ wall tube with $5 \mathrm{~g} / \mathrm{s}$, $6.5 \mathrm{~K}$ gas flowing is $0.6 \mathrm{psig}$. Since the supply and return tubing accounts for less than 2 psi pressure drop, there is approximiately 10 psi available across the control valve and cryostat. See Appendix H, page H3.

\section{VLPC Nitrogen flowrates and pressure drops}

The nitrogen tube sizes were chosen from a practical size standpoint. It is impractical to go to a size smaller than $3 / 8^{\prime \prime} \mathrm{OD}$. Based on experience with the solenoid nitrogen calculations, I conclude that pressure drop in the VLPC nitrogen piping will be negligible for the required flowrates. No formal calculations were done. 


\section{Table of Contents for the Appendices}

\section{$\underline{\text { Raw Engineering Calculations }}$}

Appendix A: Solenoid Cooldown and Flowrates etc.

Appendix B: Estimated flowrate thru Solenoid cooldown piping and delta P.

Appendix C: Solenoid pipe size: Comparison to CDF.

Appendix D: Calculation to determine VLPC Cooldown and flow rates required.

Appendix E: VLPC Piping: Total pressure drop in cooldown phase.

Appendix F: Delta P for Solenoid LN2 transfer line.

Appendix G: Solenoid transfer line steady state conditions.

Appendix H: VLPC LHe line sizing for steady state conditions.

Appendix I: Heat loads for Solenoid \& VLPC transfer lines

Appendix J: Steady state heat loads to solenoid transfer line.

Appendix K: Solenoid steady state calcs. Helium piping.

Appendix L: Solenoid steady state calcs. Helium return piping. 


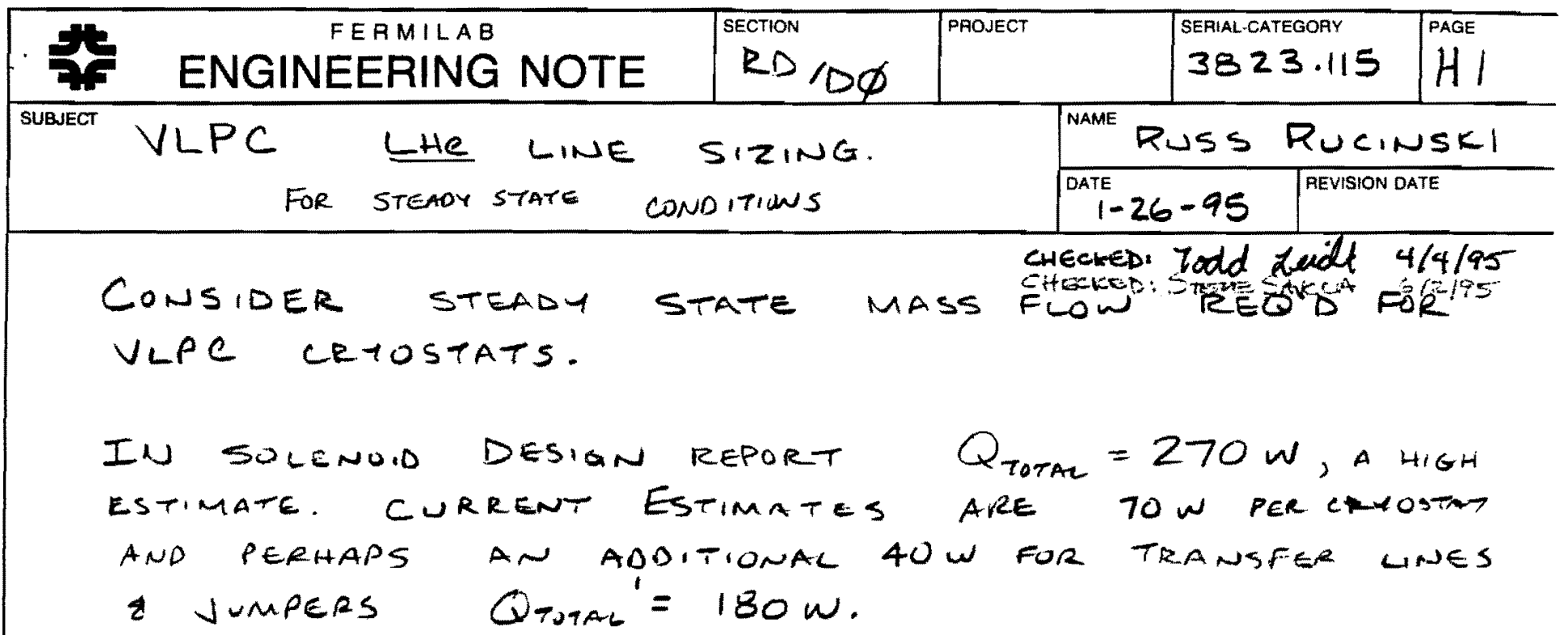

to be conservative use $270 \mathrm{w}$ number.

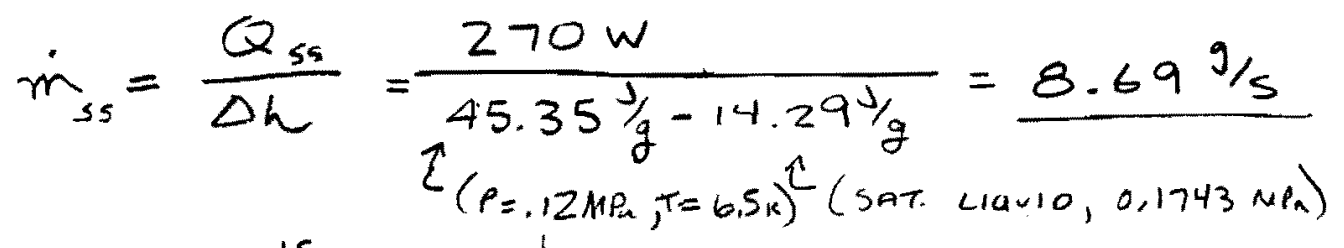

RETURN CONDITIONS

CALCULATE $\triangle P_{100}$ For $\dot{m}=4.35 \frac{9}{\mathrm{~S}} T=6.5 \mathrm{~K}, \bar{P}=.14 \mathrm{MPa}$ SAY$$
3 / 8^{11} \text { OD. } \times .035 \text { waLl, id }=.305
$$$$
R e=\frac{4 \dot{m}}{\pi D_{\mu}}=\frac{4 \cdot\left(.00435^{\mathrm{kg} / \mathrm{s}}\right)}{\pi(.305 \mathrm{~m})(.0254 \mathrm{~m} / \mathrm{N})\left(1.726 \times 10^{-6} \mathrm{~Pa}-\mathrm{s}\right)}
$$

$$
\begin{gathered}
=414,214 \quad f=.013 \\
\left.\rho=11.90 \mathrm{~kg} / \mathrm{m}^{3} \quad \Delta P=3.62 \frac{k p q^{2}}{d^{4}} \quad k=\frac{F L}{D} \quad \begin{array}{l}
\text { CRANE, } \\
E Q \cdot 3-14 \\
E Q, 2-4
\end{array}\right\}
\end{gathered}
$$$$
K_{100}=(.013) \frac{(100 \mathrm{Fe})}{\frac{.3055}{12} \mathrm{me}}=51.1
$$$$
q=\dot{m} / \rho=\frac{.00435 \mathrm{~kg} / \mathrm{s}}{11.90 \mathrm{~kg} / \mathrm{m}^{2}}=3.655 \times 10^{-4} \mathrm{~m}^{3} / \mathrm{s} \times\left(\frac{3248 \mathrm{ft}}{1 \mathrm{~m}}\right)^{3}
$$$$
=2.645 \text { sig }
$$$$
\Delta p=11.84
$$

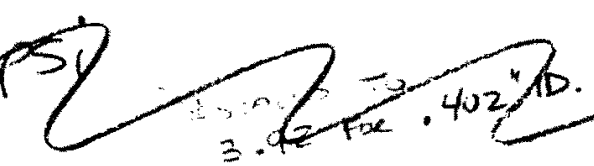




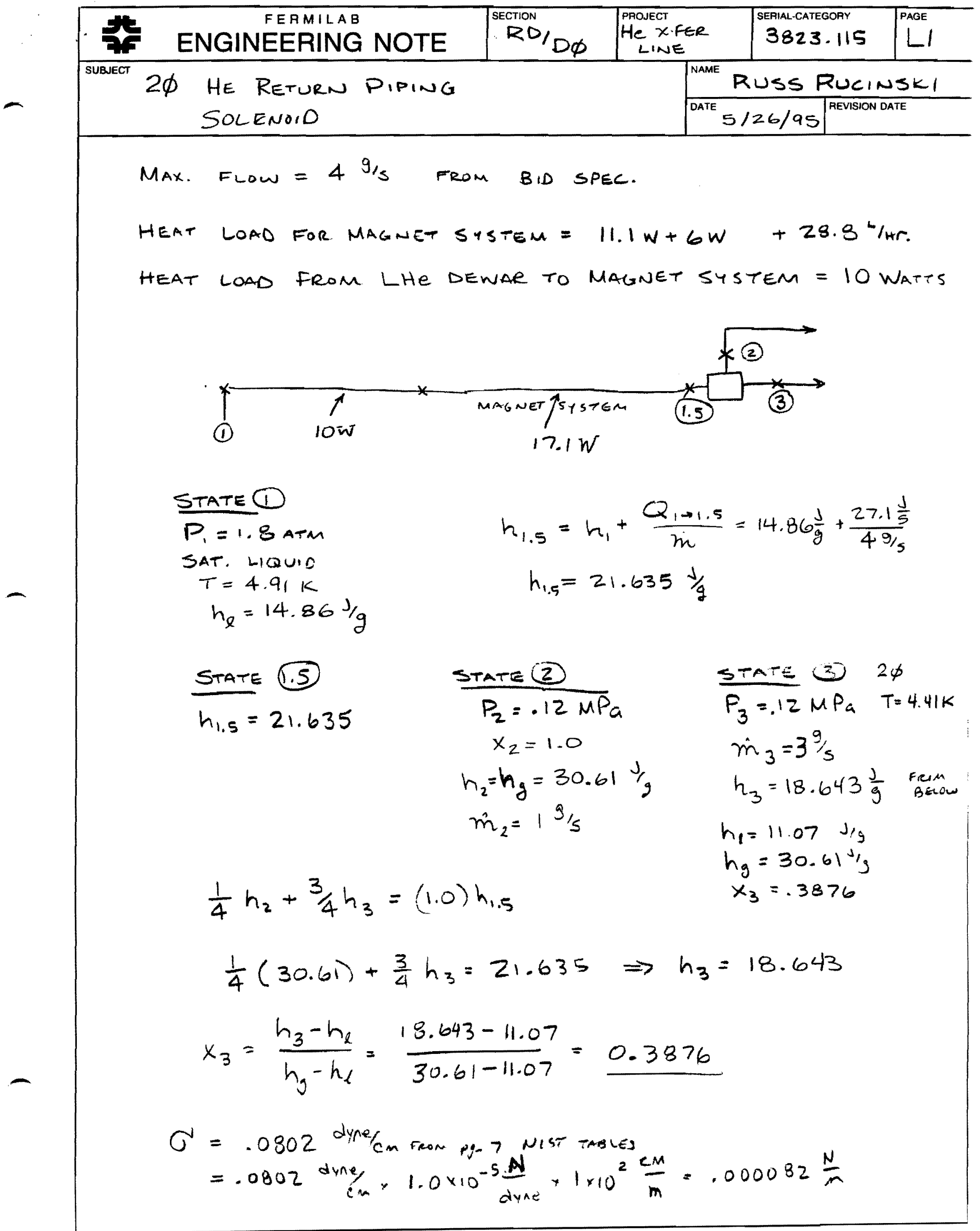




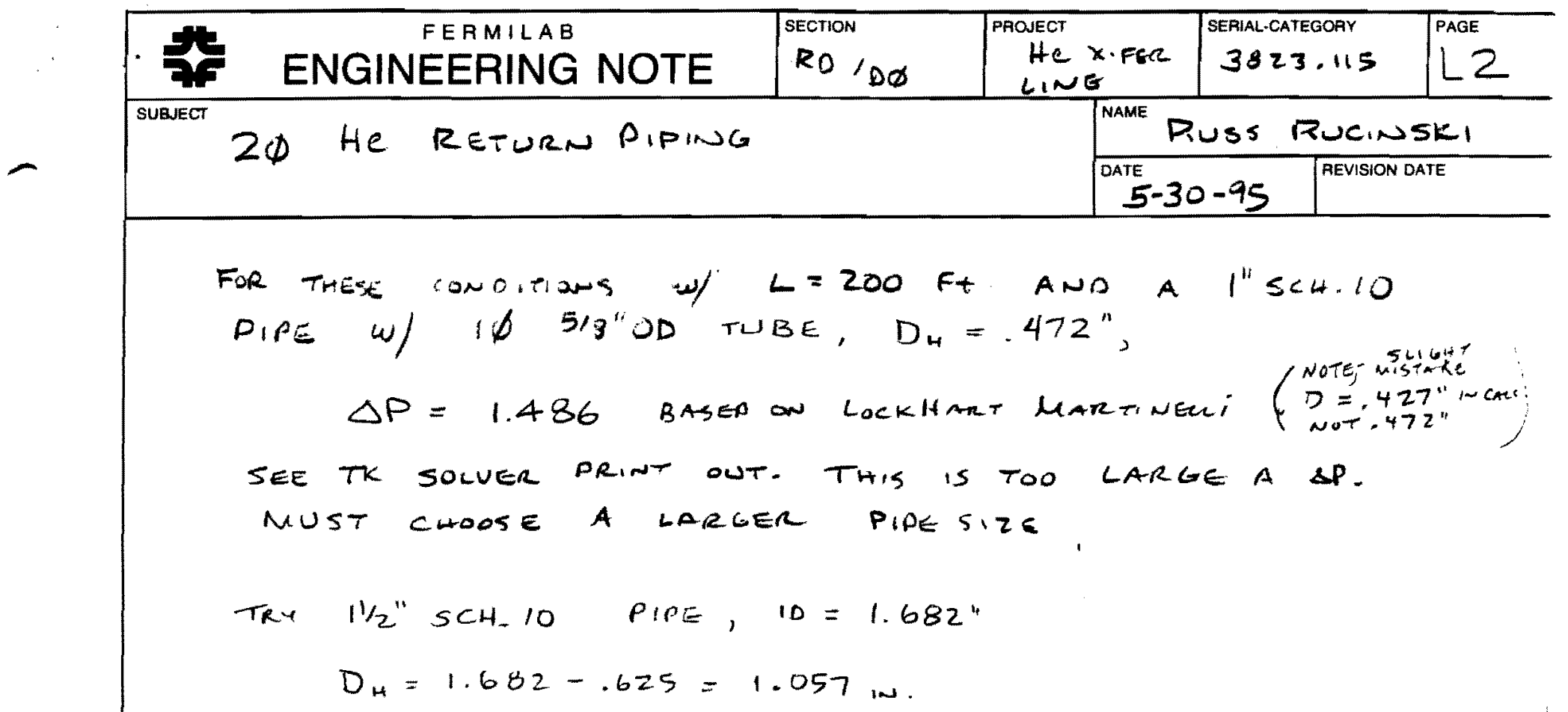

MUCH BETTER $\triangle P=.02$ psi, in WAUY/STRATIFIEO

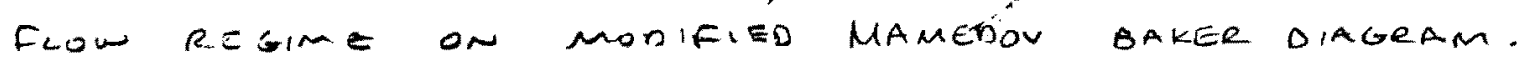
$11 / 2 "$ SCH.1O IS THE BEST SIZE. THE RADIATION SHIELO IS 3.5" OD. SO TWERE IS PLENTY OF Room for THIS SIZE. 
St Input Name output Unit $\therefore$

\begin{tabular}{|c|c|c|c|}
\hline & valid & 'accurate & \\
\hline & $\begin{array}{l}\text { flow } \\
\text { f }\end{array}$ & $\begin{array}{l}\text { 'turbulen } \\
.01906103\end{array}$ & \\
\hline .000005 & epsilon & & ft \\
\hline .427 & $D$ & & in \\
\hline 200 & $\mathrm{I}$ & & $\mathrm{ft}$ \\
\hline & $\mathrm{A}$ & $9.2387 \mathrm{E}-5$ & $\mathrm{~m}^{\wedge} 2$ \\
\hline 4 & mdot & & $g / s$ \\
\hline 121.1 & rhol & & $\mathrm{kg} / \mathrm{m}^{\wedge} 3$ \\
\hline 20.17 & rhog & & $\mathrm{kg} / \mathrm{m}^{\wedge} 3$ \\
\hline 3.18 & muI & & $\mu \mathrm{Pa}-\mathrm{s}$ \\
\hline 1.323 & muG & & $\mu \mathrm{Pa}-\mathrm{s}$ \\
\hline .000082 & sigmaI & & $\mathrm{N} / \mathrm{m}$ \\
\hline .3876 & $\mathrm{x}$ & & \\
\hline & ReL & 90430 & \\
\hline & mdotI & .0024496 & $\mathrm{~kg} / \mathrm{s}$ \\
\hline & ReG & 137600 & \\
\hline & mdotG & .0015504 & $\mathrm{~kg} / \mathrm{s}$ \\
\hline & dpdIL & 5.1012286 & $\mathrm{~Pa} / \mathrm{m}$ \\
\hline & dpdLTP & 168.10555 & $\mathrm{~Pa} / \mathrm{m}$ \\
\hline & deltap & 1.4863285 & psig \\
\hline & phil & 5.7405518 & \\
\hline & $x$ & .67244052 & \\
\hline .2 & $\mathfrak{m}$ & & \\
\hline .2 & $\mathrm{n}$ & & \\
\hline .184 & CG & & \\
\hline .184 & $\mathrm{CL}$ & & \\
\hline 20 & C & & \\
\hline & lambda & 1.4281346 & \\
\hline 1.2 & rhoair & & $\mathrm{kg} / \mathrm{m}^{\wedge} 3$ \\
\hline 998 & rhoH2O & & $\mathrm{kg} / \mathrm{m}^{\wedge} 3$ \\
\hline & sigh & 534.12427 & \\
\hline .073 & sigmaH2 & & $\mathrm{N} / \mathrm{m}$ \\
\hline 1000 & muH2O & & $\mu \mathrm{Pa}-\mathrm{s}$ \\
\hline & BakerXa & 1210 & \\
\hline & BakerYa & 8660 & $1 \mathrm{bm} / \mathrm{hr}-\mathrm{ft}$ \\
\hline & $c$ & 1 & \\
\hline & $\mathbf{M}$ & 1 & \\
\hline & RsubL & .17419928 & \\
\hline & RsubG & .82580072 & \\
\hline & Gasvel & 1.0075104 & $\mathrm{~m} / \mathrm{s}$ \\
\hline & Liqvel & 1.2568736 & $\mathrm{~m} / \mathrm{s}$ \\
\hline
\end{tabular}

\section{Conment}

Russ Rucinski 5/30/95

Return He pipe for Solenoid

1" pipe w/ 5/8" tube inside

Lockhart Martinelli correlation

for two phase pressure drop

through an adiabatic horizontal pipe

model validity

flow type

friction factor ; Must enter a guess

pipe roughness

Pipe inside diameter

Length of pipe

cross sectional area

Total mass flow rate

Liquid density

density of the gas

Iiquid viscosity

Gas viscosity

Surface tension of liquid

quality $=$ mdotG/mdot

Reynold's \# for liquid

Mass flow rate for liquid

Reynold's \# for gas

Mass flow rate for gas

$\Delta \mathrm{P} / \Delta \mathrm{L}$ for the liquid

$\Delta \mathrm{P} / \Delta \mathrm{L}$ for the two phase

Total pressure drop for the pipe $\leftarrow$ Too LARck,

Lockhart-Martinelli parameter

Lockhart-Martinelli parameter

L-M constant; Look up in Table 7.19

L-M constant; Look up in Table 7.19

L-M constant; Look up in Table 7.19

L-M constant; Look up in Table 7.19

L-M constant: Look up in Table 7.19

Baker diagram dimensionless parameter

density of air

density of water

Baker diagram dimensionless parameter

Surface tension of water

viscosity of water

$X$ - axis value for Baker plot

$Y$ - axis value for Baker plot

Volume fraction of liquid phase

Volume fraction of gas phase

Gas velocity

Liquid velocity 
Lockhart Martinell1 correlation

for two phase pressure drop

through an adiabatic horizontal pipe

\begin{tabular}{|c|c|c|c|c|}
\hline & valid & 'accurate & & model validity \\
\hline & flow & 'turbulen & & flow type \\
\hline & & .02260289 & & friction factor : Must enter a guess \\
\hline .000005 & epsilon & & ft & pipe roughness \\
\hline 1.057 & & & in & Pipe inside diameter \\
\hline 200 & I & & ft & Length of pipe \\
\hline & & .00056612 & $\mathrm{~m}^{\wedge} 2$ & cross sectional area \\
\hline 4 & mdot & & $g / s$ & Total mass flow rate \\
\hline 121.1 & rhol & & $\mathrm{kg} / \mathrm{m}^{\wedge} 3$ & Liquid density \\
\hline 20.17 & rhog & & $\mathrm{kg} / \mathrm{m}^{\wedge} 3$ & density of the gas \\
\hline 3.18 & muL & & $\mu \mathrm{Pa}-\mathrm{s}$ & Liquid viscosity \\
\hline 1.323 & muG & & $\mu \mathrm{Pa}-\mathrm{s}$ & Gas viscosity \\
\hline .000082 & sigmal & & $\mathrm{N} / \mathrm{m}$ & Surface tension of liquid \\
\hline .3876 & & & & quality $=$ mdotG $/$ mdot \\
\hline & ReL & 36530 & & Reynold's \# for Ilquid \\
\hline & mdotI & .0024496 & $\mathrm{~kg} / \mathrm{s}$ & Mass flow rate for liquid \\
\hline & $\operatorname{ReG}$ & 55580 & & Reynold's \# for gas \\
\hline & mdotG & .0015504 & $\mathrm{~kg} / \mathrm{s}$ & Mass flow rate for gas \\
\hline & dpdLI & .0650814 & $\mathrm{~Pa} / \mathrm{m}$ & $\Delta \mathrm{P} / \Delta \mathrm{L}$ for the liquid \\
\hline & dpdLTP & 2.1275204 & $\mathrm{~Pa} / \mathrm{m}$ & $\Delta P / \Delta L$ for the two phase \\
\hline & deltap & .01881077 & psig & Total pressure drop for the pipe \\
\hline & $\begin{array}{l}\text { phil } \\
\mathrm{X}\end{array}$ & $\begin{array}{l}5.7175294 \\
.67767548\end{array}$ & & $\begin{array}{l}\text { Lockhart-Martinelli parameter } \\
\text { Lockhart-Martinelli parameter }\end{array}$ \\
\hline .2 & $\mathrm{~m}$ & & & L-M constant; Look up in Table 7.19 \\
\hline .25 & $\mathrm{n}$ & & & L-M constant; Look up in Table 7.19 \\
\hline .184 & CG & & & L-M constant: Look up in Table 7.19 \\
\hline .316 & $C L$ & & & L-M constant: Look up in Table 7.19 \\
\hline 20 & C & & & I-M constant: Look up in Table 7.19 \\
\hline & lambda & 1.4281346 & & Baker diagram dimensionless parameter \\
\hline 1.2 & rhoair & & $\mathrm{kg} / \mathrm{m}^{\wedge} 3$ & density of air \\
\hline 998 & rhoH 20 & & $\mathrm{~kg} / \mathrm{m}^{\wedge} 3$ & density of water \\
\hline & sigh & 534.12427 & & Baker diagram dimensionless parameter \\
\hline $\begin{array}{l}.073 \\
1000\end{array}$ & $\begin{array}{l}\text { slgmaH2 } \\
\text { muH2O }\end{array}$ & & $\begin{array}{l}\mathrm{N} / \mathrm{m} \\
\mu \mathrm{Pa}-\mathrm{s}\end{array}$ & $\begin{array}{l}\text { Surface tension of water } \\
\text { viscosity of water }\end{array}$ \\
\hline & BakerXa & 1210 & & $x$ - axis value for Baker plot 7 see \\
\hline & BakerYa & 1410 & & y - axis value for Baker plot $\}$ sat Figure \\
\hline & c & 1 & & WANY/STRAT, \\
\hline & M & 1 & & Ecrar \\
\hline & RsubL & .17490072 & & Volume fraction of liquid phase \\
\hline & RsubG & .82509928 & & Volume fraction of gas phase \\
\hline & Gasvel & .16456003 & $\mathrm{~m} / \mathrm{s}$ & Gas velocity \\
\hline & Liqvel & .20429237 & $\mathrm{~m} / \mathrm{s}$ & Liquid velocity \\
\hline
\end{tabular}




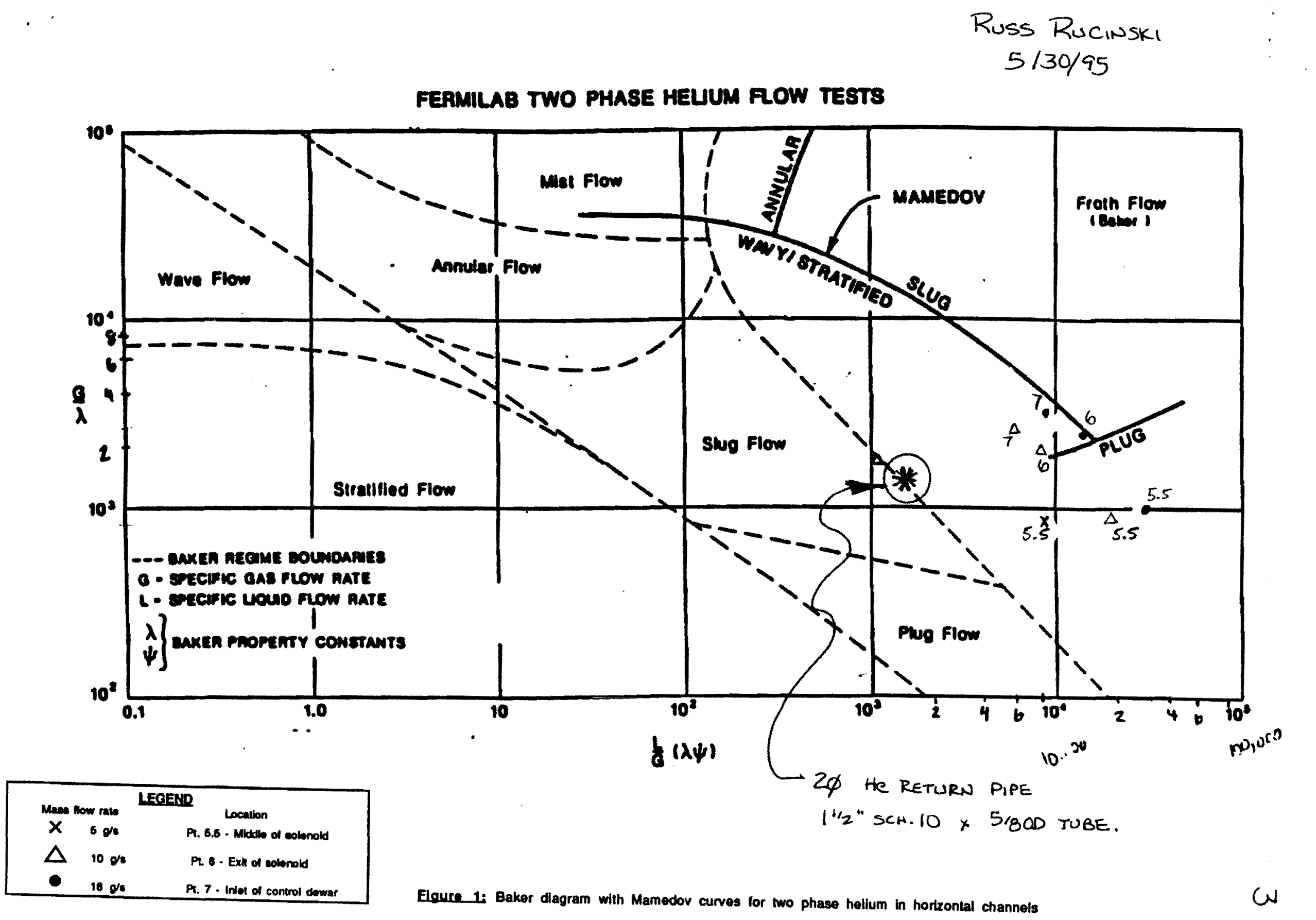

FERMILAB TWO PHASE HELUM RLOW TESTS

\section{Russ Rucinski \\ $5 / 30 / 95$}

Eloure 1: Baker dlagram with Mamedov curves tor two phase helium in horkontal channels 


\section{PIPE SIZING: \\ $\underline{\text { FOR }}$ \\ SOLENOID/VLPC \\ CRYOGENIC SYSTEMS}

\section{D-ZERO ENGINEERING NOTE \# 3823.115- EN-416 February 20, 1995}

Russ Rucinski and Steve Sakla $\mathrm{RD} / \mathrm{D} \emptyset$ Mech.

Approved : 


\section{Introduction}

The addition of a solenoid magnet and VLPC detectors are two of a number of upgrades which will occur at the D-Zero detector in the near future. Both of these upgrades will require cryogenic services for their operation. The purpose of this engineering note is to document the pipe/tube size choices made for these cryogenic services. This was done by calculating the required flow rates to cool down the magnet and VLPC's over a reasonable length of time and to determine the required piping sizes for a given allowable pressure drop. The pressure drops for steady state conditions also are addressed. The cool down requirements drove the pipe size decision. The raw engineering calculations that were done for this project are included as an appendix to this note. The body of this document discusses the methods and results of the calculations. As a quick summary, Figures 1 and 2 show the size selections. Tables 1 and 2 give a more detailed size and description of each section of Solenoid and VLPC transfer line.

\section{VIPC Detector/Cryostat Description}

The main detecting component of the VLPC detector is the VLPC (Visible Light Photon Counter) chip. These chips have an optimum performance at a temperature of about $6.5 \mathrm{~K}$ which will be maintained through the use of a cryostat. Two cryostats, named east and west, will be used to maintain the cold temperatures of the chips through the use of liquid helium. Liquid nitrogen will be used to intercept some of the heat leak coming in to the cryostat. These cryogens will be supplied to the cryostat via a vacuum jacketed transfer line and $u$-tubes. The transfer lines begin at the LHe refrigerator location near the southeast comer of the D-Zero cleanroom. The VLPC supply and return lines share a common vacuum jacketed line. These lines will originate at the valve box on the refrigerator side and will run along the south wall, see figure 4 . The building side route for the VLPC line follows the same path as the solenoid, see figure 5. The detector side VLPC piping can be seen in figure 6. For more description see the D0 engineering note "Cryogenic Line routing: Refrigerator to VLPC Cryostats and Solenoid", D0 note \# 3823.115-EN-413.

\section{Solenoid Magnet Description}

The solenoid magnet will provide a 2 tesla magnetic field in the inner bore of the central calorimeter. It will operate at approximately $4.9 \mathrm{~K}$ which will be obtained by means of indirect cooling from liquid helium. As with the VLPC cryostat liquid nitrogen 


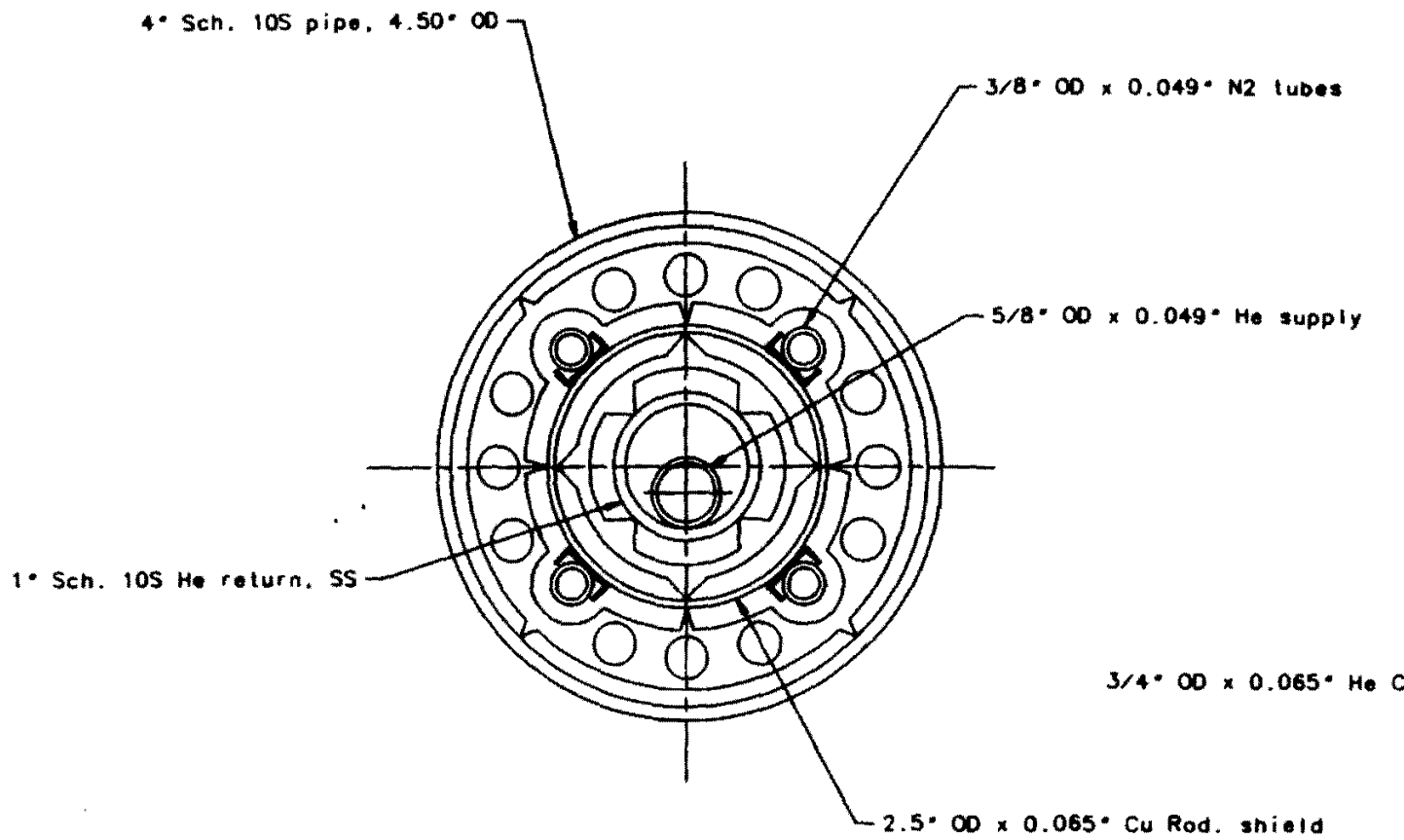

Moin Solenoid $x$ fer line

Solenoid xfer I ine

PREL IMINARY BUILDING

TRANSFER LINE DESIGNS

Rusa Rucinski 2/21/9s

$$
\text { Figure } 1
$$


Table 1 : saenoid presections

\begin{tabular}{|c|c|c|c|c|c|c|c|c|c|c|c|c|}
\hline LIME - & EHUIR & pescaenom & tras & mon & Io & $\begin{array}{c}\text { PUEE ORTUBE } \\
\text { STIE }\end{array}$ & $\begin{array}{l}\text { Neros } \\
\text { DAandu. }\end{array}$ & $\begin{array}{c}\text { LWEAB } \\
\text { LEKSTHIES) }\end{array}$ & $\begin{array}{c}\text { COE } \\
\text { ELPOWS }\end{array}$ & $\begin{array}{c}\text { LEES } \\
\text { IHAUBWW }\end{array}$ & $\begin{array}{l}\text { IEES } \\
\text { IHAU } \\
\text { BRAMCH }\end{array}$ & YALYES \\
\hline 4 & LEE & SUPPLY (HUT3) & U-TUBE & LUE DEWAR & X.FER LINE & $3 / 4^{\prime \prime}$ OD X .035 & 0.68 & 5 & 2 & 0 & 0 & 0 \\
\hline 5 & LHE & SUPPLY & X-FER LNE & U-TUBE & U-TUBE & $588^{\circ}$ oD $\times .049$ & 0.527 & 125 & 9 & 4 & 0 & I DIVRTR. \\
\hline 8 & LHE & SUPPLY (HUTA) & U-TUBE & X-FER LNE & OOMTROL DEWAR & $3 / \mathrm{A}^{\prime \prime} \mathrm{OD} \times .035$ & 0.68 & 10 & 2 & 0 & 0 & 0 \\
\hline 7 & LHE & RETURN (HUTS) & U.TUBE & CONIROL DEWAR & X-FER LNE & $3 / 4$, OD X X.035 & 0.68 & 10 & 2 & 0 & 0 & 0 \\
\hline 8 & LEE & RETURN & X-FER LNE & U-TUBE & U.TUBE & Concentrit & 0.427 & 125 & 9 & 4 & 0 & $1 \mathrm{PV}, 1 \mathrm{CV}$ \\
\hline 9 & HE & RETURN & U-TUBE & X-FER LNE & X-FER LINE (VLPC & $3 / 4^{\prime \prime}$ OD $\times .035$ & 0.68 & 3 & 2 & 0 & 0 & 0 \\
\hline 10 & LN2 & SUPPLY & U. TUBE & LIN HEADER & X.FER LNE & $1 / 22^{\prime \prime}$ OD $\times .035$ & 0.43 & 3 & 2 & 0 & a & 0 \\
\hline 11 & LN2 & SUPPLY & X-FER LINE & U-TURE & TE & $1 / 22^{\prime \prime}$ OD $\times .049$ & 0.402 & 1 & 1 & 0 & 0 & 0 \\
\hline 12 & $\mathrm{LN}_{2}$ & SUPPLY (SHIELD) & X.FER LINE & TEE & U-TURE & $3 / 8^{\prime \prime}$ oD X.049 & 0.277 & 124 & 9 & 2 & 1 & I pv \\
\hline 13 & LN2 & SUPPLY (SHIELD) & U.TUBE & X-FER LINE & OONTROL DEWAR & $1 / 2^{n}$ OD $\times .035$ & 0.43 & 10 & 2 & 0 & 0 & 0 \\
\hline 14 & LN2 & \begin{tabular}{|l} 
SUPPLY \\
(INTERCEPT)
\end{tabular} & X.FER LNE & He: & U.TUBE & $3 / 8^{\circ}$ OD X.049 & 0.277 & 124 & 9 & 2 & 1 & I p \\
\hline 15 & $\mathrm{LN}_{2}$ & \begin{tabular}{|l} 
SUPPLY \\
(INTERCEPT) \\
\end{tabular} & U.TUBE & $X$. FER LINE & DONTROLLIE:WAR & $1 / 2^{\prime \prime} \mathrm{OD} \times .035$ & 0.43 & 16 & 2 & 0 & 0 & 12 \\
\hline 16. & LN2 & RETURN (SHIELU) & U. TUBE & CONIROL DEWAR & X-FER LINE: & $1 / 2=00 \times .035$ & 0.43 & 111 & 2 & 11 & $=$ & ." \\
\hline 17 & $\mathrm{LN2}$ & RETURN (SHIELD D & X.FER LINE & U. TURE & U.TUBE & $3 / 8 "$ OD $\times .049$ & 0.277 & 12.5 & $y$ & 2 & 0 & 1 \\
\hline 18 & $\mathrm{LN}_{2}$ & RETURN (SHIELD) & U.TUBE & X-FER LINE & TEE & $1 / 2^{\prime \prime} O D \times .035$ & 0.43 & 1 & 1 & o & 1 & 11 \\
\hline 19 & LN2 & $\begin{array}{l}\text { RETURN } \\
\text { (INTERCEPT) }\end{array}$ & U.TUEE & CONIROL DE:WAR & X-FER I.WNE: & $1 / 2^{\prime \prime} 00 \times .035$ & $0 .+3$ & in & 2 & a & "1 & \\
\hline 20 & I.N2 & \begin{tabular}{|l} 
RETURN \\
(INTFRCEPT)
\end{tabular} & X.FERINV: & (1:-113: & 11.NIB: & $188^{\circ}$ OD $\times$ & 0.277 & 125 & 4 & 2 & is & \\
\hline 22 & LN2 & $\begin{array}{l}\text { RETURN } \\
\text { (INTERCEPT) }\end{array}$ & U.TUBE & X.FER LINE & $\pi x$ & $1 / 2^{\prime \prime} 0 D \times .035$ & 0.43 & 1 & 1 & $n$ & 1 & 1 \\
\hline 23 & I.N2 & RETURN & U-TUBE & IEE & GRVVENT HEADER & $1 / 2 "$ OD $\times .035$ & 0.43 & 5 & 1 & 0 & 0 & 0 \\
\hline 24 & HE & $\mid \begin{array}{l}\text { (COOLDOWN } \\
\text { QUENCH) }\end{array}$ & U.TUBE & CONIROL DEWAR & X.FER LINE & $3 / 4^{\circ}$ OD X.035 & 0.68 & 10: & 2 & a & a & 11 \\
\hline 25 & HE & $\begin{array}{l}\text { (COOLDOWN } \\
\text { QUENCH) }\end{array}$ & X-FER LINE & U-TUBE: & U. TUBE & $3 / 4^{*}$ OD X .06s & 0.62 & 125 & 9 & 2 & 0 & $n$ \\
\hline 26 & HE & $\begin{array}{l}\text { (COOLDOWN } \\
\text { QUENCH) }\end{array}$ & U.TUBE & $X$-FER LINE & $\begin{array}{l}\text { HECOOLDOWN } \\
\text { RETURN }\end{array}$ & $3 / 4^{\prime \prime}$ oD $\times$.035 & 0.68 & 5 & 2 & a & 11 & 1 \\
\hline 27 & $\mathrm{HE}$ & $\mid \begin{array}{l}\text { suPPLY } \\
\text { (COOLDOWN) }\end{array}$ & |U-TUBE & COOL.DOWN LNE & X.FER LINE & $3 / 4^{\prime \prime}$ OD $\times$, B13 & 0.68 & 5 & & a & "it & $1 \mathrm{BM}$ \\
\hline
\end{tabular}


TABLe 2 : npocplpe sections

\begin{tabular}{|c|c|c|c|c|c|c|c|c|c|c|c|c|}
\hline LINE : & ELUIR & Posaceumenon & fres & Exa & Te & \begin{tabular}{|c|} 
PIPEOR TUAE \\
:UAE \\
\end{tabular} & DIANDE & 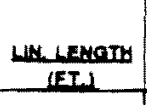 & ELOE & $\begin{array}{c}\text { IIEFa } \\
\text { TIBU BUM }\end{array}$ & $\begin{array}{l}\text { IEES } \\
\text { IHEU } \\
\text { BBANCH }\end{array}$ & YALYES \\
\hline 38 & re & $\operatorname{sen} y$ & unee & Inxo6 & ANFEEDCAN & $12^{\prime \prime} 00 \times .049$ & 0.402 & 5 & 2 & 0 & 0 & e \\
\hline 38 & WE & Sempir & unae & MEE DEWAR & ANFEEOCAN & $1 / 2^{\prime \prime} 00 \times .049$ & 0.402 & 5 & 2 & 으 & a & 0 \\
\hline 40 & $E$ & WEST HE SUPFLY & $X+E R$ UNE & uTree & 3-WAY VALVE & $3 / 8=00 \times 035$ & 0305 & 2 & 1 & $\perp$ & 0 & 0 \\
\hline 4 & $\forall$ & wert anary & - & twax & 3 war & & & $z$ & \pm & + & 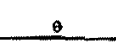 & 0 \\
\hline 42 & HE & WEST HE SUPAY & XfER UNE & IMAYV VALVE & unee & $3 / 8.00 \times 035$ & 0305 & 123 & 9 & 3 & ․ & $10 \mathrm{v}$ \\
\hline 43 & IE & MEST HESUPAYY & une & CHFEEOCAN & garonet CAN & $38 \mathrm{~g}^{\circ}$ O0 $\times 035$ & 0.305 & 12 & 2 & 0 & 은 & 0 \\
\hline 44 & IE & WEST MESUPAY Y & $X+Q R$ UKE & EAYONET CAN & Valve BoX & $3 / 8.00 \times .035$ & 0.305 & 47 & 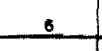 & 1 & $\underline{-}$ & - \\
\hline 45 & LE & WEST HESURPYY & $x$ tanure & VALVE BOX & npe & $318.00 \times .035$ & 0.305 & 24 & 2 & 0 & 0 & 0 \\
\hline 48 & He & EASTHESUPAY & X+E UNE & UTUEE & 3-WAY YALVE & $3 / 8^{*} 00 \times 035$ & 0.305 & 2 & 0 & 1 & 1 & 0 \\
\hline 47 & IE & EAST HE SUPALY & $x$ fER UNE & unee & 3-WAY VALVE & $318.00 \times 035$ & 0.305 & 2 & 0 & 0 & 1 & 0 \\
\hline 40 & HE & ENSTressupay & $x+$ QRUNE & 3-WAY VALVE & unex & $38.00 \times .035$ & 0.305 & 123 & 2 & 3 & 2 & lov \\
\hline 40 & Ie & EASTIESSPPVY & unee & CATEEDCN & EAYONER CAN & $318.00 \times 035$ & 0.305 & 12 & 2 & 0 & 0 & 0 \\
\hline 50 & HE & EASTHE SUPAYY & $x \in$ R UNE & BAYONET CAN & Valve Box & $38^{\circ} \mathrm{O} 0 \mathrm{x} \times 035$ & 0.305 & 4 & 3 & 1 & 0 & 0 \\
\hline 51 & HE & EAST KESEPAY & $X$ XER UNE & VALVE BOX & nec & $3 / 8^{\circ} 00 \times 025$ & 0.305 & 24 & 2 & 0 & 0 & 0 \\
\hline 52 & HE & EAST VERETUNAN & $X$ EER LIME & vipe & VALVE BOX & $316^{\circ} .00 \times 035$ & 0.305 & 24 & , & 으 & 0 & $18 \mathrm{PV}$ \\
\hline 53 & NE & EASTEERERUAN & $X$ XER UNE & VAUVEEOXX & BAYONET CAN & $318.00 \times 035$ & 0.305 & 47 & 5 & 0 & 0 & 0 \\
\hline 54 & HE & EAST HERERUPN & unes & BAYONET CAN & OMFEDOCN & $38.00 \times .035$ & 0.305 & 12 & 2 & 0 & 0 & 0 \\
\hline 55 & HE & EAST HE RETURN & $x+x_{1}$ un & CW FEEDCAN & 3WAY VAVE & $318.00 \times .035$ & 0.305 & 123 & 0 & 3 & 1 & $10 y$ \\
\hline 56 & re & EASTHERETLRN & $x+E$ LIE & 3WAY VALVE & $\begin{array}{l}\text { WJ LNE ICOOLOOWN } \\
\text { RETUAN }\end{array}$ & $38.00 \times .035$ & 0.305 & 2 & 2 & 1 & 1 & $10 \mathrm{~V}$ \\
\hline 57 & 位 & EASTIERERUAN & $x+$ terune & 3-WAY VALVE & VJLUNE (NALVE BOX) & $318.00 \times .035$ & 0.305 & 2 & 2 & $\underline{2}$ & 1 & $1 \mathrm{cV}$ \\
\hline 58 & EE & WEST LERERLAN & XPR LuE & voc & VNUVE BOX & $38.00 \times 035$ & 0.305 & 24 & 1 & 0 & 0 & IPV \\
\hline 58 & IE & WESTIE RERURN & $X$ XFUE & VALVE BOX & BAYONET CAN & $3 / B=00 \times 035$ & 0.305 & 47 & 5 & 0 & 0 & 0 \\
\hline 30 & $E$ & WESTHERERURN & unes & MAYONET CAN & CAHEDOCN & $30.00 \times 005$ & 0.305 & 12 & 2 & 0 & 0 & 0 \\
\hline 91 & IE & HESTHE RERRAN & $x+R$ UNE & CH FEED CAN & 3-WAY VALVE & $38.00 \times .035$ & 0.305 & 123 & 2 & 2 & 0 & $10 \mathrm{~V}$ \\
\hline 62 & HE & WEST HERERUAN & $X$ XERUE & 3-WAY VALVE & $\begin{array}{l}\text { VU LUNE ICOOLDOWN } \\
\text { RERUPMI }\end{array}$ & $3 / 00 \times 0.035$ & 0.305 & 2 & 2 & 2 & 2 & $1 \mathrm{CV}$ \\
\hline as & EE & MEST HE RERUAN & $x+$ EunE & 3.WAY VALVE & VS UNE NNVVE BOX) & $3 / 10.00 \times 005$ & 0.305 & \pm & 1 & 0 & 2 & $\mathrm{ICV}$ \\
\hline 84 & HE' & WEST HERETURN & $x+$ LWE & 3-WAY VALVE & FE & $280.00 \times .035$ & 0.305 . & 2 & 1 & 1 & 2 & $10 \mathrm{~V}$ \\
\hline 8.5 & HE & PETUN & $x+$ ERUNE & $\begin{array}{l}\text { UTVRE FACM SOL } x \text { - } \\
\text { PERLEE }\end{array}$ & TEE & $12^{*} O D \times .049$ & 0.402 & 1 & 1 & 2 & 1 & 0 \\
\hline 60 & 트 & nerlen & Whes & NHFEER CAN & $\begin{array}{l}\text { OoOLDOMN RETUAN } \\
\text { UNE }\end{array}$ & $1 / 2=00 \times .049$ & 0.402 & 5 & 2 & 0 & 0 & 0 \\
\hline 67 & HE & henew & UTEE & AHFEEDCN & VALVE BoX & $34.00 \times .035$ & 0.08 & 4 & 0 & 0 & a & 0 \\
\hline o & LNR & sumy & UneE & werenos & NHFEED CNN & $388^{\circ}, 00 \times$.049 & 0.277 & 3 & 2 & 0 & 0 & 0 \\
\hline se & LNR & sumpry & XFGR UNE & NHFEEDCN & CHFEEOCN & $3: 8^{n}$ OD $\times$ and & 0.277 & 125 & 9 & 2 & 0 & 0 \\
\hline 70 & $w_{2}$ & Sumpry & unas & COMEDOAN & BAYONET CAN & $3 / 8^{\prime \prime}$ OD X $\times 049$ & 0.277 & 12 & 2 & 0 & 0 & 0 \\
\hline 71 & $\omega_{2}$ & sempry & $x+E$ un: & Gavoner CAN & VALVE BOX & $3 / 8 \times O D \times .049$ & 0.277 & 47 & 6 & 1 & 0 & 0 \\
\hline 72 & LN2 & sumpy & $x+E R$ UNE & VAVEEOX & YPC WEST & $3 / 8 " O D \times \quad .049$ & 0.277 & 24 & 3 & 1 & 1 & IPV \\
\hline 73 & une & suppy & Xfor & VAVEE COX & I NPCEAST & $3 / 8^{\prime \prime}$ OD $\times \quad .049$ & 0.272 & 24 & 3 & 1 & 1 & IPV \\
\hline 24 & $w_{2}$ & sumpry & $x+$ fens: & vecuest & VALVE EOX & $3 / 8^{*}$ OD $\times .049$ & 0.277 & 24 & 0 & 0 & 1 & 0 \\
\hline 75 & UR & semery & XPQR LNE & IVPCEAST & VALVE $90 X$ & $3 / 8=$ OD X & 0.277 & 24 & 1 & 1 & 0 & 0 \\
\hline 76 & $\mathrm{~N}_{2}$ & sumy & $x$ xpune & VASVE BOX & EAYONET CAN & $3 / 8=$ OD $\times$.049 & 0.277 & 47 & 5 & 1 & 0 & 0 \\
\hline 7 & $L_{2}$ & Denar & UnEe & Baronet Can & loH FEDOCAN & $3 / 8^{\prime \prime}$ OD $\times .049$ & 0.277 & 12 & 2 & 0 & 은 & 0 \\
\hline 78 & $L_{2}$ & RETRE & $x+g$ uN & OUPEDCA & NWFEEDCN & $3 / 8=00 \times .049$ & 0.277 & 125 & 0 & 2 & 0 & 0 \\
\hline 70 & WN2 & Penne: & Lunee & NHFEEDCAN & GR VENT HEAOER & $3,8 "$ oD X 0.04 & 0.277 & 5 & 2 & 0 & 0 & 0 \\
\hline & cs & ITME & II & & & & & & & & & \\
\hline & & & & & & & & & & & & \\
\hline
\end{tabular}




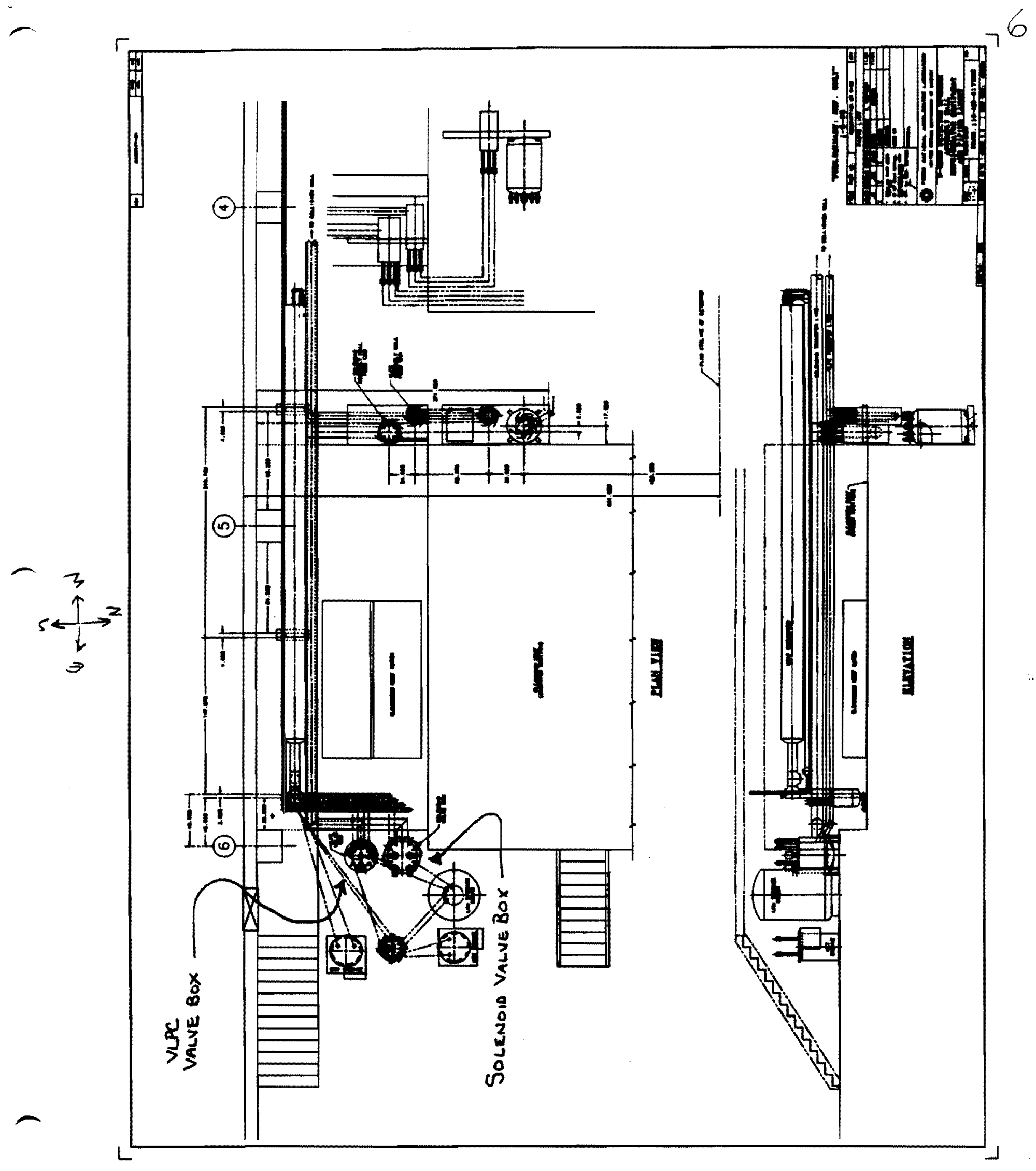

FIGURE 4 


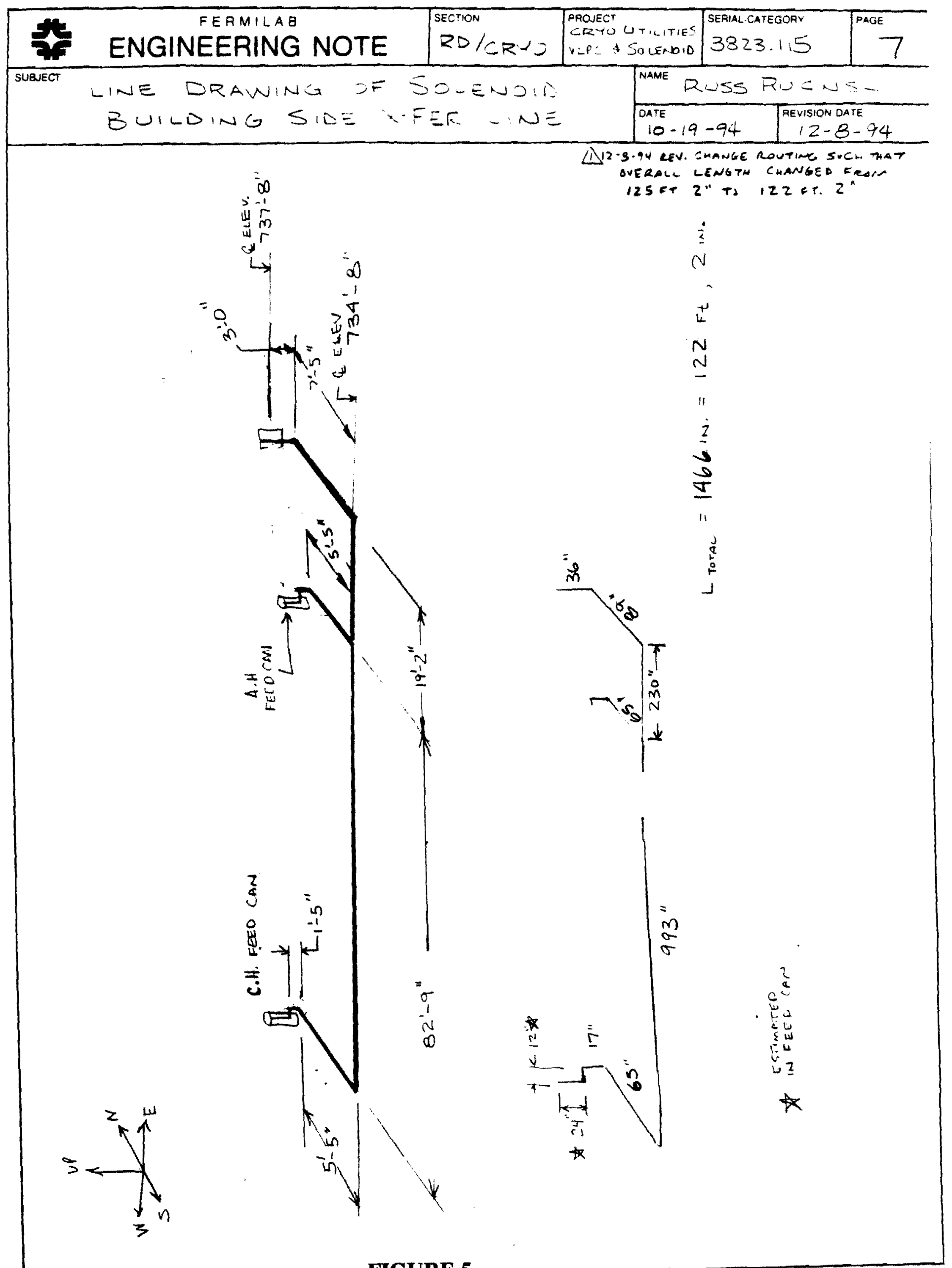




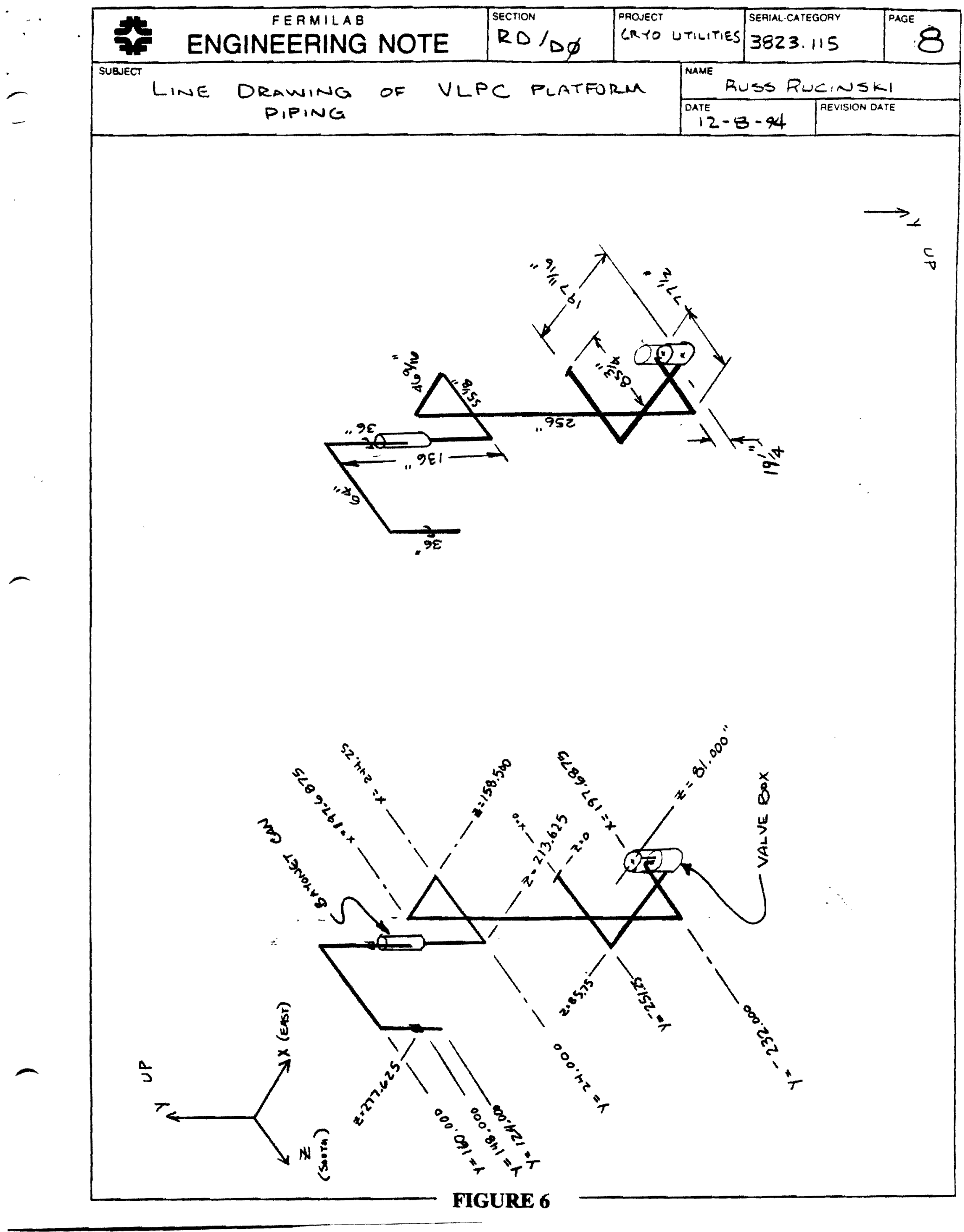


intercepts will be used. Along with liquid helium the liquid nitrogen will be supplied via a vacuum jacketed transfer line and u-tubes. These line will essentially follow the same path as the VLPC piping (figure 4) in the assembly hall A line drawing of the solenoid building side transfer line can be seen in figure 5 . Via u-tubes the transfer line connects to the control dewar mounted on the detector. The routing path on the detector is described in chapter 5 of the solenoid design report (Fermilab-TM-1886) and is also shown graphically in figure 7. The helium supply and return lines in the transfer line will consist of two concentric pipes. The supply line will run inside the return line to make use of the enthalpy of the return cold gas. Preliminary Flow schematics for both the VLPC and solenoid piping (FNAL Drg. 3823.115-ME-317223) are shown in figures 8 thru 10.

\section{Solenoid Helium Cooldown flowrates}

The LHe line sizes for the solenoid were determined by first calculating the required flow rates during cooldown. These calculations are shown in "Solenoid Cooldown \& Flow Rates Etc." of appendix A and are summarized in Table 3. A cooldown rate of $5 \mathrm{~K} / \mathrm{hr}$ was used in these calculations. It should be noted that it is planned to limit the actual rate to $2 \mathrm{~K} / \mathrm{hr}$.

Table 3: SOLENOID COOLDOWN FLOW RATES

\begin{tabular}{|c|c|c|c|c|c|c|}
\hline STEP & $\begin{array}{c}\text { SOLENOW } \\
\text { TEMP } \\
{[\mathbf{K}]}\end{array}$ & $\begin{array}{c}\text { GAS } \\
\text { TEMP } \\
\text { [N [K] }\end{array}$ & $\begin{array}{c}\text { GAS } \\
\text { TEMP } \\
\text { OUT [K] }\end{array}$ & $\begin{array}{c}\text { TIME } \\
\text { [hrs] }\end{array}$ & $\begin{array}{c}\text { DELTA H } \\
\text { COLD MASS } \\
{[\mathbf{J}]}\end{array}$ & $\begin{array}{c}\text { MASS FLOW } \\
\text { RATE REQ'D } \\
{[\mathrm{g} / \mathrm{s}]}\end{array}$ \\
\hline 1 & 300 to 250 & 200 & 250 & 10 & $6.42 \mathrm{E}+07$ & 6.9 \\
\hline 2 & 250 to 200 & 150 & 200 & 10 & $6.05 \mathrm{E}+07$ & 6.5 \\
\hline 3 & 200 to 150 & 100 & 150 & 10 & $5.44 \mathrm{E}+07$ & 5.8 \\
\hline 4 & 100 to 90 & 80 & 90 & 4 & $6.59 \mathrm{E}+06$ & 8.8 \\
\hline
\end{tabular}

The mass flow rates of steps $1 \& 4$ were the largest. The largest pressure drop of the steps was determined by noting that $\triangle P \alpha \frac{f L W^{2}}{\rho d^{5}}$. The corresponding pressure drops were ratioed to determine which was larger. It was concluded that step 1's pressure drop would be about 2.6 times larger than step 4's. Therefore the cooldown lines should be sized for a flow rate of $7 \mathrm{~g} / \mathrm{s}$ with an inlet temperature of $200 \mathrm{~K}$ and an outlet temperature of $300 \mathrm{~K}$ (refer to appendix A). 


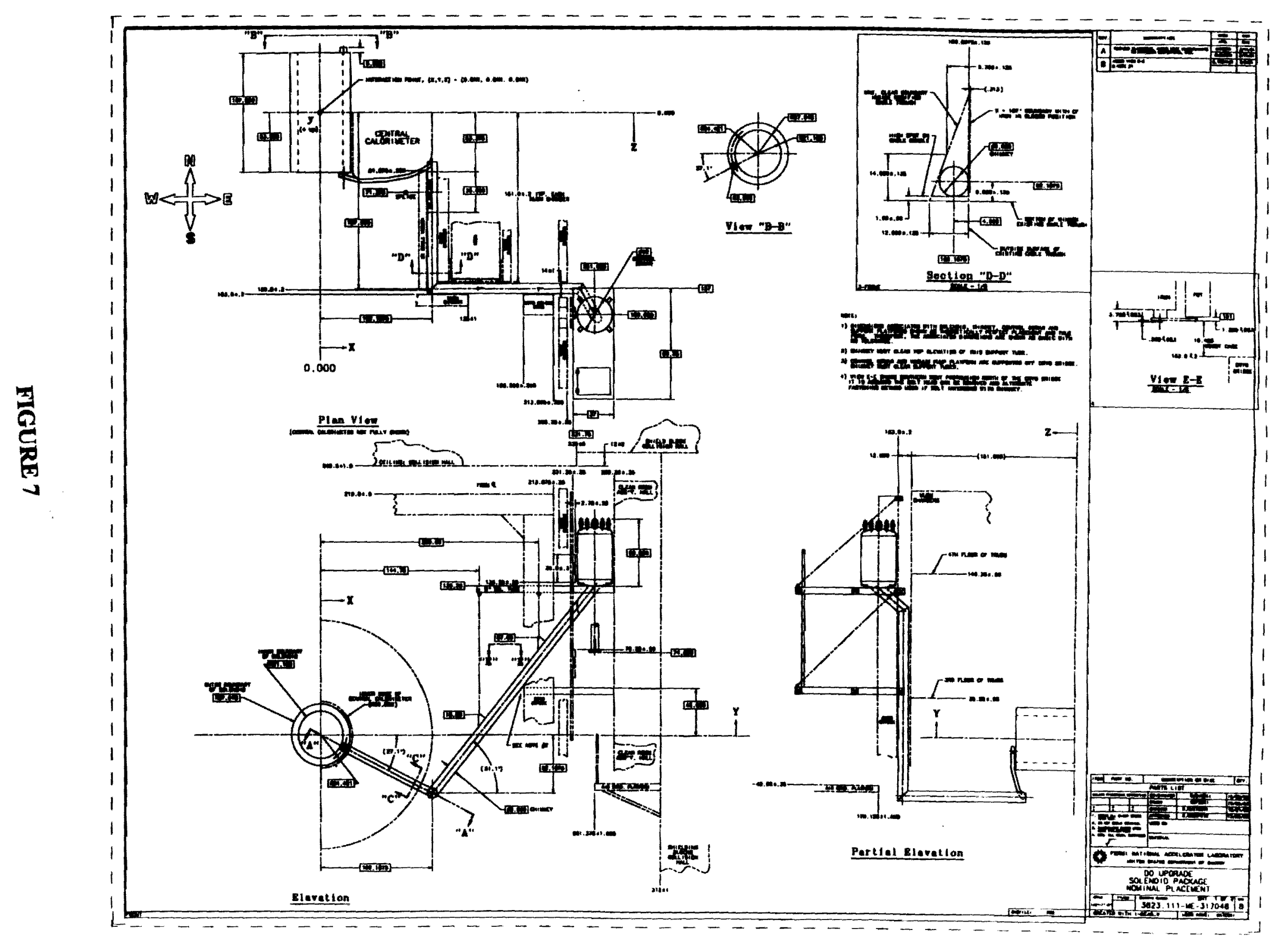




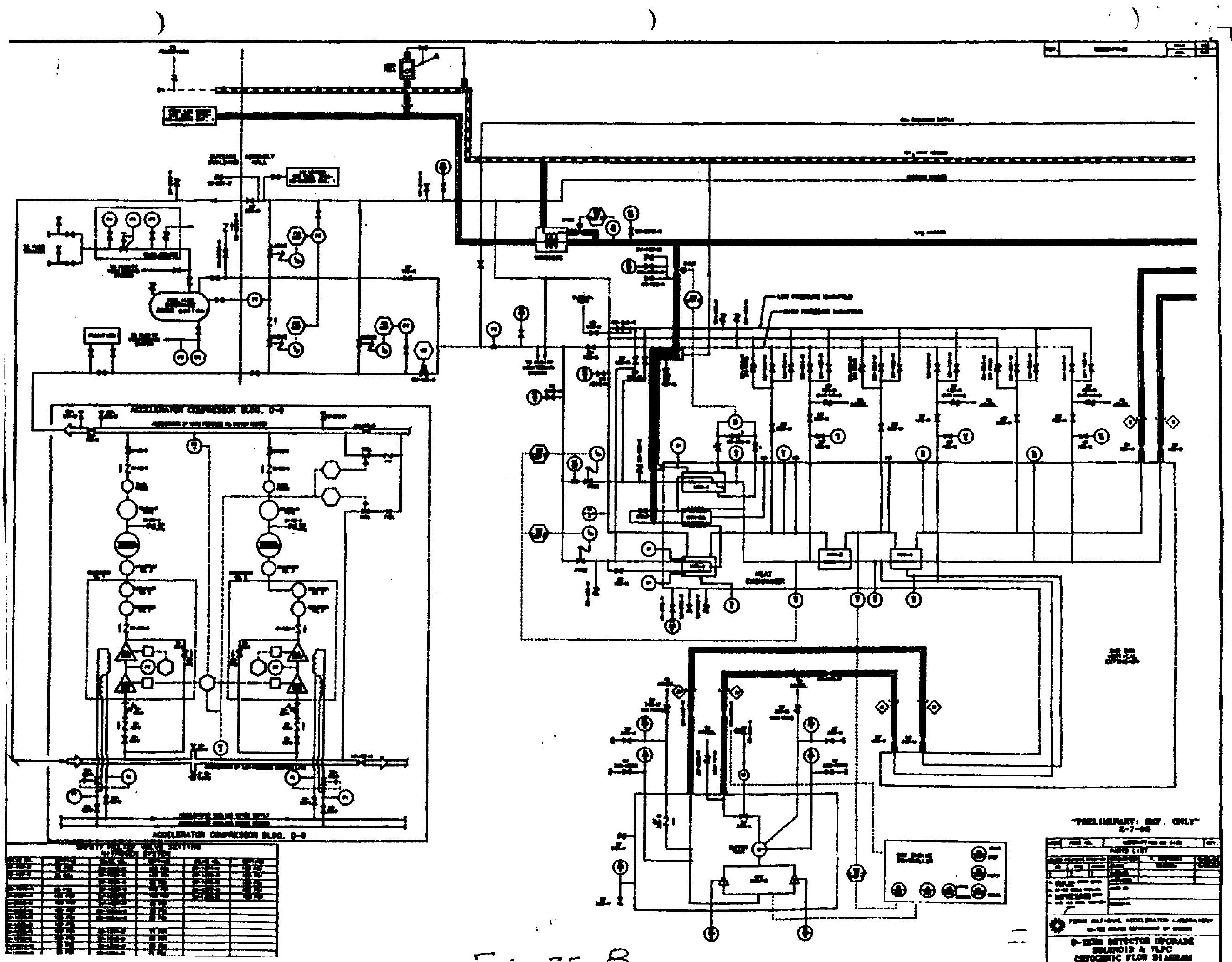


For comparison, D-Zero engineering note \#3823.111-EN-359 documents more complex calculations for a cooldown rate of $2 \mathrm{~K} / \mathrm{hr}$. The highest mass flow for this cooldown rate was $4.4 \mathrm{~g} / \mathrm{s}$ as shown in 3823.111-EN-359. (See table 4 below). Therefore, the flow rate of $7 \mathrm{~g} / \mathrm{s}$ used to size the cooldown lines will be a conservative number.

\begin{tabular}{|c|c|c|c|c|c|c|c|}
\hline Step & $\begin{array}{c}\text { Inlet } \\
\text { Temperature } \\
\text { [Kelvin] }\end{array}$ & $\begin{array}{c}\text { Exit } \\
\text { Temperature } \\
\text { [Kelvin] }\end{array}$ & $\begin{array}{c}\text { Iniet } \\
\text { Enthaipy } \\
{[\mathrm{J} / \mathrm{O}]}\end{array}$ & $\begin{array}{c}\text { Exit } \\
\text { Enthalpy } \\
{[\mathrm{J} / \mathrm{a}]}\end{array}$ & \begin{tabular}{|c} 
Inlet \\
Pressure \\
[psig|
\end{tabular} & $\begin{array}{c}\text { Exit } \\
\text { Pressure } \\
\text { fosigl }\end{array}$ & $\begin{array}{l}\text { Mass } \\
\text { Flow } \\
|g / s|\end{array}$ \\
\hline 1 & 175 & 275 & 925 & 1444 & 18.9 & 5 & 1.2 \\
\hline 2 & 125 & 225 & 665 & 1184 & 7.7 & 5 & 1.2 \\
\hline 3 & 80 & 175 & 431 & 924 & 6.5 & 5 & 1.1 \\
\hline 4 & 80 & 125 & 431 & 665 & 7.7 & 5 & 1.9 \\
\hline 5 & 80 & 95 & 431 & 509 & 13.8 & 5 & 4.4 \\
\hline 8 & 4.6 & 80 & 30.2 & 431 & 7 & 5 & 2.3 \\
\hline 7 & 4.6 & 60 & 30.2 & 327 & 7 & 5 & 2.7 \\
\hline 8 & 4.6 & 45 & 30.2 & 249 & 7 & 5 & 3.3 \\
\hline 9 & 4.6 & 35 & 30.2 & 197 & 7 & 5 & 3.8 \\
\hline 10 & 4.6 & 27.5 & 30.2 & 158 & 7 & 5 & 4.4 \\
\hline 11 & 4.6 & 22.5 & 30.2 & 132 & 7 & 5 & 4.7 \\
\hline 12 & 4.6 & 17.5 & 30.2 & 105 & 7 & 5 & 5.5 \\
\hline 13 & 4.6 & 12.5 & 30.2 & 78.6 & 7 & 5 & 6.7 \\
\hline 14 & 4.6 & 8.75 & 30.2 & 58.0 & 7 & 5 & 8.3 \\
\hline 15 & 4.6 & 6 & 30.2 & 41.8 & 7 & 5 & 10.4 \\
\hline
\end{tabular}

\section{Solenoid Helium Cooldown Pressure drops}

The first step in performing these calculations was to generate a list of the various piping sections along with their corresponding lengths and number of elbows, tees, and valves. Each pipe section was given a number. See table 1 for a listing of the solenoid pipe sections.

Pressure drops due to friction, valves, tees, bends, etc. were then calculated for the given pipe sizes and inlet/exit states. The solenoid cooldown piping was first sized by assuming a pressure drop of $95 \mathrm{psig}$, an inlet temperature of $200 \mathrm{~K}$ GHe and an outlet temperature of $300 \mathrm{~K}$ through the supply and return lines respectively. Resistance coefficients were calculated for each piping section and for the solenoid. Each coefficient was converted to correspond to an inner diameter of 0.68 in. since the i.d.'s of each section were not all the same. In the second step of the calculation, pressure drops were calculated for each individual pipe section taking into account the change in density due to changes in pressure. Summing the individual pressure drops resulted in a total pressure drop of $36.3 \mathrm{psi}$. These numbers are summarized in table 5. 
Table 5: Solenoid Helium Cooldown piping pressure drop summary

\begin{tabular}{|c|c|c|c|c|c|c|c|}
\hline $\begin{array}{c}\text { PIPE } \\
\text { SECTION }\end{array}$ & $\begin{array}{c}\text { INLET } \\
\text { TEMP } \\
(\mathbf{K})\end{array}$ & $\begin{array}{c}\text { OUTLET } \\
\text { TEMP(K) }\end{array}$ & $\begin{array}{c}\text { INLET } \\
\text { PRESS } \\
\text { (PSIA) }\end{array}$ & $\begin{array}{c}\text { OUTLET } \\
\text { PRESS } \\
\text { (PSIA) }\end{array}$ & $\begin{array}{c}\text { MASS } \\
\text { FLOW } \\
\text { (GS) }\end{array}$ & $\begin{array}{c}\text { RESISTANCE } \\
\text { COEFFICIENT (K) })\end{array}$ & $\begin{array}{c}\text { PRESSURE DR (PSIA) } \\
\text { DROP }\end{array}$ \\
\hline LINE 27 & 200 & 200 & 114.7 & 114.58 & 7 & 12.2 & 0.12 \\
\hline LINE 5 & 200 & 200 & 114.58 & 107.88 & 7 & 194.92 & 6.7 \\
\hline LINE 6 & 200 & 200 & 107.88 & 107.69 & 7 & 5.2 & 0.19 \\
\hline SOLENOID & 200 & 300 & 107.69 & 86.09 & 7 & 430.2 & 21.6 \\
\hline LINE 24 & 300 & 300 & 86.09 & 85.72 & 7 & 5.44 & 0.37 \\
\hline LINE 25 & 300 & 300 & 85.72 & 79.61 & 7 & 90.84 & 6.11 \\
\hline LINE 26 & 300 & 300 & 79.61 & 79.35 & 7 & 3.41 & 0.26 \\
\hline $\begin{array}{c}\text { COOLDOWN } \\
\text { RETURN }\end{array}$ & 300 & 300 & 79.35 & 78.38 & 7 & 13 & 0.97 \\
\hline & & & & & & & \\
\hline & & & & & & TOTAL DELTA P $=$ & $\mathbf{3 6 . 3}$ pSi \\
\hline
\end{tabular}

It was estimated that using the total acceptable pressure drop of $95 \mathrm{psi}, 200 \mathrm{~K}$ in, $300 \mathrm{~K}$ out, that the maximum cooldown flow rate obtainable would be $11.3 \mathrm{~g} / \mathrm{s}$.

Comparison to CDF pipe sizes: A simple comparison to CDFs pipe sizes was made. The calculations are in appendix C. The D-Zero solenoid cold mass is one-fourth that of CDF's. The cooldown rate for both solenoids will be the same so D-zero will only need one-fourth the mass flow rate during cooldown. For the same allowable pressure drop at one-fourth the flow the CDF pipe sizes could be reduced by 0.33 . D-Zero's pipe sizes are compared to this number and are found to be larger.

Table 6: Comparison to CDF sizes

\begin{tabular}{|l|l|l|l|}
\hline LINE SECTION & dCDF & $0.33^{*}$ dCDF & dD-Zero \\
\hline He supply & $1.00^{\prime \prime}$ OD & $0.33^{\prime \prime}$ & $0.625^{\prime \prime}$ OD \\
\hline He return & $1.00^{\prime \prime}$ OD & $.33^{\prime \prime}$ & $0.75^{\prime \prime}$ OD \\
\hline LN2 supply & $0.5^{\prime \prime}$ OD & $.17^{\prime \prime}$ & $0.375^{\prime \prime}$ OD \\
\hline N2 return & $0.625^{\prime \prime}$ OD & $.21^{\prime \prime}$ & $0.375^{\prime \prime}$ OD \\
\hline
\end{tabular}




\section{Solenoid Helium Steady State Pressure drops}

Some preliminary calculations were done to estimate the steady state helium heat loads, flowrates and pressure drops expected for each section of piping. See appendices $\mathrm{I}, \mathrm{J}$, and $\mathrm{K}$. The total pressure drop from the tubing sizes were on the order of 3.2 psi as shown in the summary table in appendix $\mathrm{K}$, page $\mathrm{K} 12$. Essentially all this pressure drop occurred in the long return pipe, line \# 8 . The pressure drop was based on line \# 8 being a concentric tube with hydraulic radius of $0.347^{\prime \prime}$. This calculation was never given a final check or revised because it was decided that the cooldown process determined the required pipe sizes. It should be noted that the current size selection (Table 1 and figure 1) give line \# 8 a larger hydraulic radius of $0.427 "$. Therefore the helium steady state pipe pressure drops will be negligible.

\section{Solenoid Nitrogen flowrates and pressure drops}

The nitrogen flowrate requirements for cooldown and steady state are considered in calculations in appendix F. The maximum pressure drop allowed for the nitrogen circuit inlet to outlet is $30 \mathrm{psi}$, based on the current storage dewar operating pressure. Per the Solenoid bid spec., the required nitrogen cooldown flowrate shall not exceed $5 \mathrm{~g} / \mathrm{s}$. With the current selected pipe sizes nitrogen flow will less be than $5 \mathrm{~g} / \mathrm{s}$ until the returm piping fluid is less than $150 \mathrm{~K}$. This means initially we may cannot deliver the full $5 \mathrm{~g} / \mathrm{s}$. I feel this is acceptable however. The pressure drop for steady state nitrogen requirements is expected to be less than 1 psi (with control valves full open). See appendix $\mathrm{G}$.

\section{VLPC Helium Cooldown flowrates}

The LHe line sizes for the VLPC were determined by first calculating the required flow rates during cooldown. These calculations are shown in "VLPC Cooldown \& Flow Rates Etc." of appendix D and are summarized in Table 7. A cooldown rate of $5 \mathrm{~K} / \mathrm{hr}$ was used in these calculations.

Table 7: VLPC COOLDOWN FLOW RATES

\begin{tabular}{|c|c|c|c|c|c|}
\hline STEP & $\begin{array}{c}\text { VLPC } \\
\text { TEMP } \\
\text { [K] }\end{array}$ & $\begin{array}{c}\text { GAS } \\
\text { TEMP } \\
\text { IN [K] }\end{array}$ & $\begin{array}{c}\text { GAS } \\
\text { TEMP } \\
\text { OUT [K] }\end{array}$ & $\begin{array}{c}\text { TIME } \\
\text { [hrs] }\end{array}$ & $\begin{array}{c}\text { MASS FLOW } \\
\text { RATE REQ'D } \\
\text { [g/s] }\end{array}$ \\
\hline 1 & 300 to 250 & 200 & 250 & 10 & .84 \\
\hline 2 & 250 to 200 & 150 & 200 & 10 & .89 \\
\hline 3 & 200 to 150 & 100 & 150 & 10 & .92 \\
\hline 4 & 100 to 90 & 80 & 90 & 4 & 3.1 \\
\hline
\end{tabular}


It was concluded that step 4's pressure drop would be about 3.6 times larger than step 1's. Therefore the cooldown lines were sized for a flow rate of $3.1 \mathrm{~g} / \mathrm{s}$ with an inlet temperature of $80 \mathrm{~K}$ and an outlet temperature of $90 \mathrm{~K}$ (refer to appendix D).

\section{VLPC Helium Cooldown Pressure drops}

A list of the various piping sections along with their corresponding lengths and number of elbows, tees, and valves. Each pipe section was given a number. See table 2 for a listing of the VLPC pipe sections.

Pressure drops due to friction, valves, tees, bends, etc. were then calculated for the given pipe sizes and inlet/exit states. The VLPC cooldown piping was first sized by assuming a pressure drop of $95 \mathrm{psig}$, an inlet temperature of $80 \mathrm{~K} \mathrm{GHe}$ and an outlet temperature of $90 \mathrm{~K}$ through the supply and retum lines respectively. Resistance coefficients were calculated for four segments of the piping. In the second step of the calculation, pressure drops were calculated for each segment taking into account the change in density due to changes in pressure. Summing the individual pressure drops resulted in a rough estimated pressure drop of 59.1 psi. These numbers are summarized in table 8 . See Appendix $\mathrm{E}$ for the raw calculations.

Table \& VLPC Helium Cooldown piping pressure drop summary

\begin{tabular}{|c|c|}
\hline PIPE SECTION & PRESSURE DROP (PSIA) \\
\hline Supply line 38 & 0.44 \\
\hline West supply lines + VLPC cryostat & 27.4 \\
\hline West return lines & 28.1 \\
\hline Return line 66 & 3.2 \\
\hline Total Delta P $=$ & 59.1 psi \\
\hline
\end{tabular}

\section{VLPC Helium Steady State Pressure drops}

The expected pressure drop for $3 / 8$ " OD $\times 0.035$ wall tubing is less than $0.1 \mathrm{psi}$ per 100 feet of tubing. See appenix $\mathrm{H}$. No further calculations were done. 


\section{VLPC Nitrogen flowrates and pressure drops}

The nitrogen tube sizes were chosen from a practical size standpoint. It is impractical to go to a size smaller than $3 / 8^{\prime \prime} \mathrm{OD}$. Based on experience with the solenoid nitrogen calculations, I conclude that pressure drop in the VLPC nitrogen piping will be negligible for the required flowrates. No formal calculations were done. 


\section{Table of Contents for the Appendices}

\section{Raw Engineering Calculations}

Appendix A: Solenoid Cooldown and Flowrates etc.

Appendix B: Estimated flowrate thru Solenoid cooldown piping and delta P.

Appendix C: Solenoid pipe size: Comparison to CDF.

Appendix D: Calculation to determine VLPC Cooldown and flow rates required.

Appendix E: VLPC Piping: Total pressure drop in cooldown phase.

Appendix F: Delta P for Solenoid LN2 transfer line.

Appendix G: Solenoid transfer line steady state conditions.

Appendix H: VLPC LHe line sizing for steady state conditions.

Appendix I: Heat loads for Solenoid \& VLPC transfer lines

Appendix J: Steady state heat loads to solenoid transfer line.

Appendix K: Solenoid steady state calcs. Helium piping. 


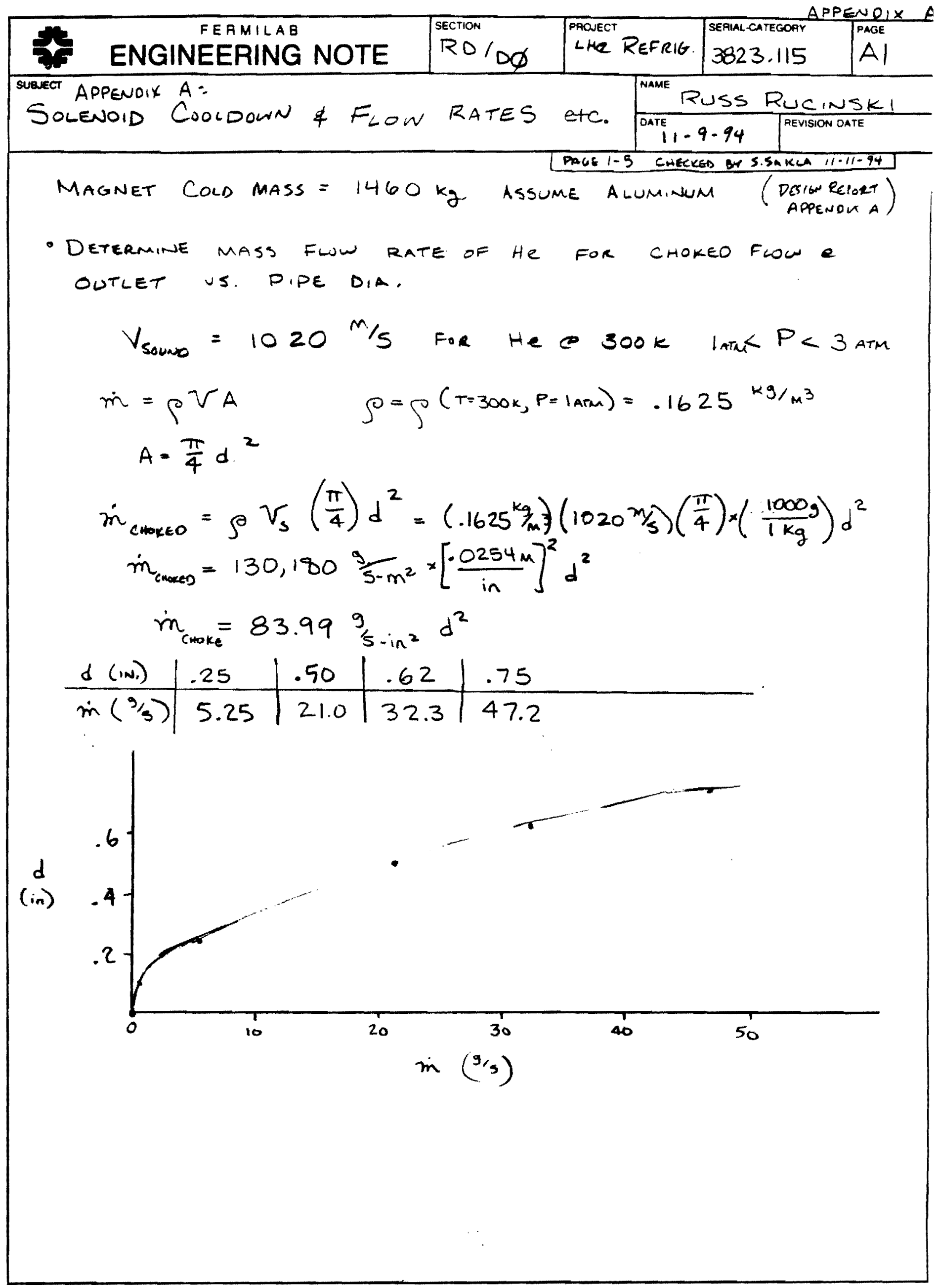




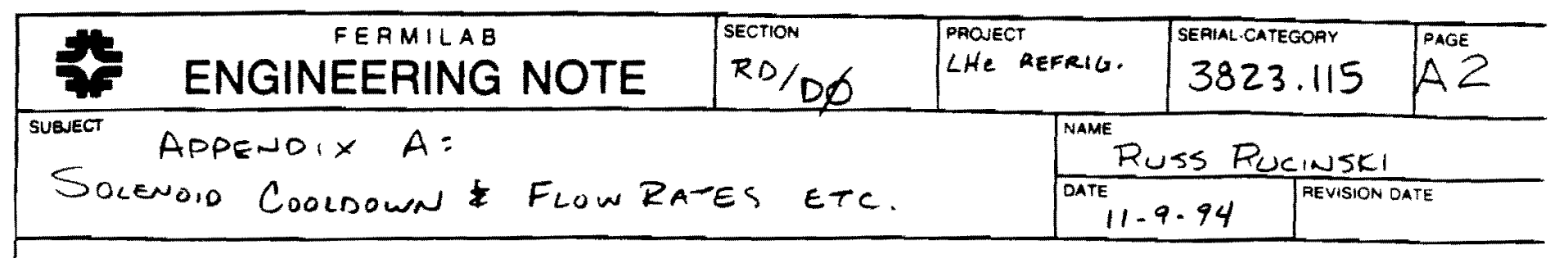

LOOK \& MAXIMUM COOLDOWN FLOW RATE REaD.

SAY $d T_{d t_{\text {max }}}=5 \mathrm{k} /$ HR fOR SOLEnOID.

* Note: This is a rough analysis not iterated to 2 place accuracy.

STEP 1

$300 k$ to $250 \mathrm{~K}$ in 10 Hours.

$$
\begin{aligned}
& \Delta H_{\text {can }}=n \Delta h=1460 \mathrm{~kg}\left[170.4 \frac{\mathrm{g}}{\mathrm{g}}-126.4 \frac{\mathrm{l}}{\mathrm{g}}\right]\left[\frac{1000 \mathrm{~g}}{1 \mathrm{~kg}}\right] \\
& \Delta \underset{\substack{\text { mass } \\
\text { dos }}}{H_{0}}=6.424 \times 10^{7} \mathrm{~J}
\end{aligned}
$$

SAY $200 K$ GAS IN, 250K GAS OUT

$$
\begin{aligned}
& h_{\text {IN }}=1054 \frac{\mathrm{J}}{\mathrm{g}} \quad h_{\text {OUT }}=1314 \mathrm{~J} / \mathrm{g} \quad \rho(p=0.2 \mathrm{MPa}, 1=225 \mathrm{~K})=.028 \frac{\mathrm{lbm}}{\mathrm{ft}^{3}}
\end{aligned}
$$

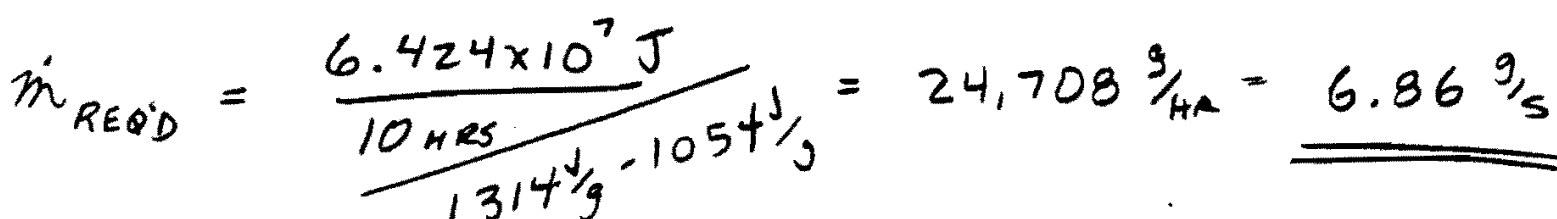

Check avg heat lond

$$
\bar{Q}=\frac{6.424 \times 10^{7} \mathrm{~J}}{10 \mathrm{HRs}} \times \frac{1 \mathrm{me}}{3600 \mathrm{~s}}=1784 \mathrm{~W}
$$

CHECK AU L CONnECTION COFF.

$$
\bar{h}=\frac{\bar{Q}}{A \Delta T_{\text {mo r }}}=\frac{1784 \mathrm{~W}}{\pi(.015 \mathrm{~m})(60.4 \mathrm{~m})(50 \mathrm{~K})}=12.5 \mathrm{~W} / \mathrm{m}^{2} \cdot \mathrm{K}
$$

SeEms to be a reasonable convection coefficient EASILY OBTAINABLE FOR TURBULENT FLOW

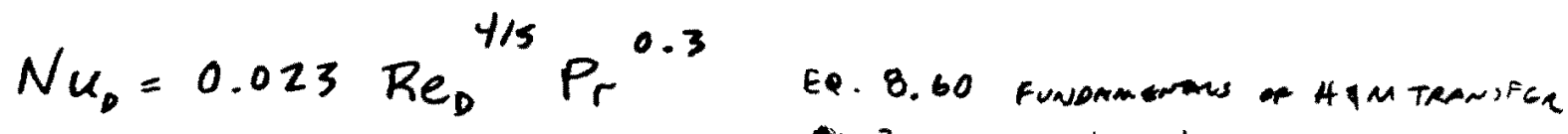

$$
\begin{aligned}
& \operatorname{Re}_{D}=22,700 \frac{\mathrm{gP}}{d \mu} \approx 22,700 \frac{\left(.5 \times \frac{\mathrm{ft}^{3}}{\mathrm{hr}^{3}}\right)\left(.028^{\mathrm{bm} / \mathrm{t}^{3}}\right)}{(.59 \mathrm{~m})\left(1.6 \times 10^{-2} \mathrm{cp}\right)} \approx \frac{3.6 \times 10^{4}}{\text { Rovotiot }} \\
& \operatorname{Pr} \approx .66
\end{aligned}
$$




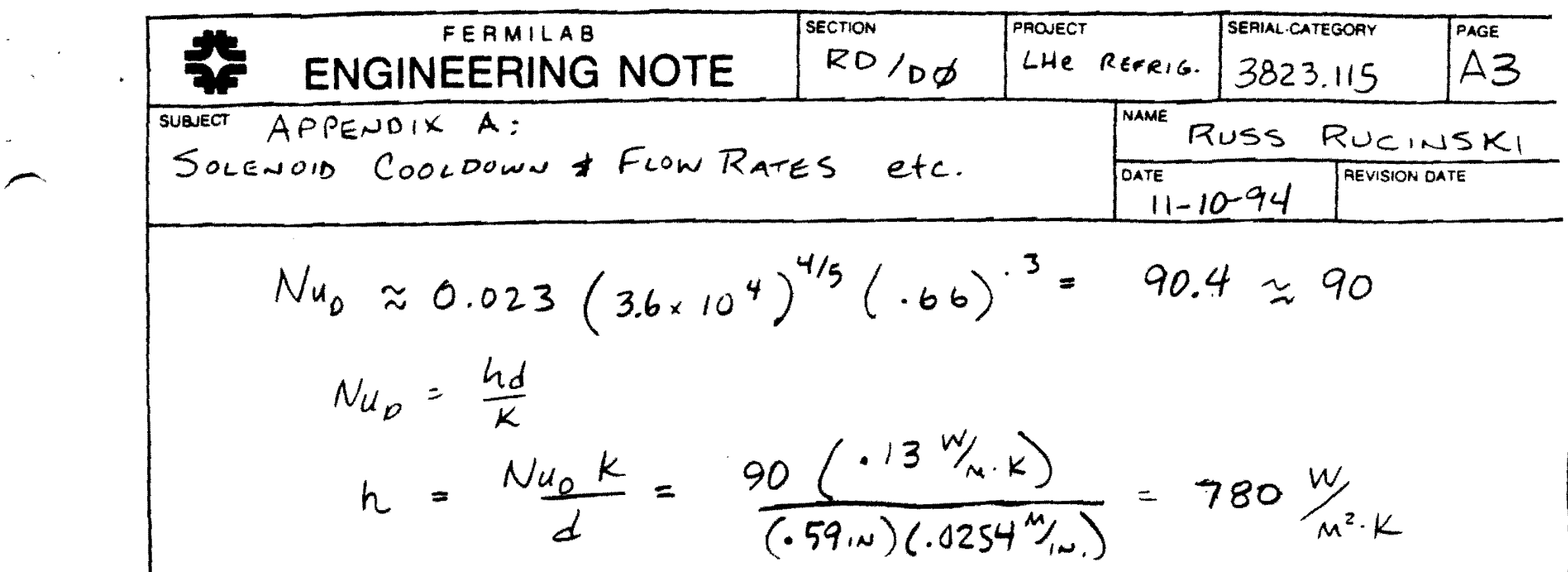

Conclusion: We have plenty of heat transfer area AND COEFFICLNT to COOLDOWN AT RATE OF $5 \mathrm{~K} / \mathrm{H}$.

STEP 2

$$
\begin{aligned}
\Delta \|_{\text {cows }} & =m \Delta h=1460 \mathrm{~kg}\left[126.4 \frac{\mathrm{J}}{\mathrm{g}}-84.8 \frac{\mathrm{J}}{\mathrm{g}}\right]\left[\frac{1000 \mathrm{~g}}{1 \mathrm{~kg}}\right] \\
& =6.074 \times 10^{7} \mathrm{~J}
\end{aligned}
$$

SAY $150 \mathrm{~K}$ GAS IN, $200 \mathrm{~K}$ GAS OUT

$$
\begin{aligned}
& h_{\text {iN }}=795 \mathrm{~J} / \mathrm{g} \quad h_{\text {OUT }}=1055 \mathrm{~J} / \mathrm{g} \quad \text { nOTE VALUGS for } p=-2 \mathrm{MPa} \\
& \dot{m}_{\text {REdO }}=\frac{6.074 \times 10^{7} \mathrm{~J}}{(10 \mathrm{HRS})\left(1055 \frac{\mathrm{H}}{\mathrm{g}}-795 \mathrm{~J} / \mathrm{g}\right)} \times \frac{1 \mathrm{HR}}{3600 \mathrm{~s}}=6.5 \mathrm{~g} / \mathrm{s}
\end{aligned}
$$

Heat load \& convection cofF. REQ'D wILl be similar to step 1.

STEP 3

$$
\begin{aligned}
& 200 \mathrm{~K} \text { to } 150 \mathrm{~K} e 5 \mathrm{k} / \mathrm{Hr} \\
& \Delta H_{\text {cos }}=m \Delta h=1460 \mathrm{~kg}\left[84.8 \frac{\mathrm{J}}{\mathrm{g}}-47.55 \frac{\mathrm{J}}{\mathrm{g}}\right] \times \frac{1000 \mathrm{~g}}{1 \mathrm{Kg}}=5.44 \times 10^{7} \mathrm{~J}
\end{aligned}
$$

SAY TOOK GAS IN, $150 \mathrm{~K}$ GAS OUT

$$
\begin{gathered}
h_{\text {IN }}=535.1 \mathrm{~J} / \mathrm{g} \quad h_{\text {OUT }}=795 \mathrm{~J} / \mathrm{g} \\
\dot{m}_{\text {REQ D }}=\frac{5.44 \times 10^{7} \mathrm{~J}}{(10 \text { MRS })\left(795 \frac{\mathrm{J}}{\mathrm{g}}-535.1 \mathrm{~J} / \mathrm{g}\right)(3600 \mathrm{~s})}=5.81 \mathrm{~g} / \mathrm{s}
\end{gathered}
$$




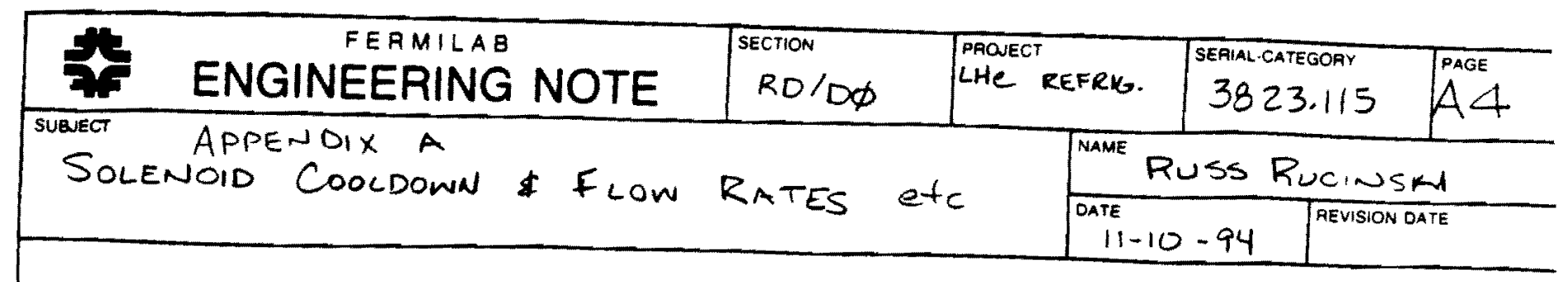

Required mass flow rates are going along same DIRECTION IN TIME STEPS AS B. SQUIRES COOCDOWN NOTE EN-359. MY FLOW RATES ARE ROUGHLY 5.4 TIMES GREATER DUE TO INCREASED COOLDOWN RATE Conservative $\triangle T$ of Gas of 5OK.

LOOK AT ONE OF WORST STEPS, 5 100K TO $90 \mathrm{~K}$.

STEP 4

SOLENOiD TEMP. $100 \mathrm{~K}$ TS $90 \mathrm{~K}$

ALLOW 4 HES FOR THIS STEP

$$
\Delta H_{\text {cons }}=1460 \mathrm{~kg}\left[17.76 \frac{\mathrm{J}}{\mathrm{g}}-13.25 \frac{\mathrm{J}}{\mathrm{g}}\right] \times \frac{1000 \mathrm{~g}}{1 \mathrm{~kg}}=6.585 \times 10^{6} \mathrm{~J}
$$

SAY BOKGAS IN, $90 \mathrm{~K}$ GAS OUT

$$
\begin{aligned}
& h_{\text {w }}=431.2 \frac{\mathrm{J}}{\mathrm{g}} \quad h_{\text {out }}=483.2 \mathrm{~J} / \mathrm{g} \\
& \dot{m}_{R E O^{\prime} O}=\frac{6.585 \times 10^{6} \mathrm{~J}}{(4 \text { eRS })(483.2 \mathrm{~J} / \mathrm{g}-431 / \mathrm{y})} \times \frac{1 \mathrm{HR}}{3600 \mathrm{~s}}=8.8 \mathrm{~g} / \mathrm{s} \\
& \bar{Q}=6.585 \times 10^{6} \mathrm{~J} / 4 \text { HeS } \times 3600 \mathrm{~s} / \mathrm{HR}=457 \mathrm{~W} \\
& \bar{h}_{\text {REQ }} \cdot \frac{\bar{Q}}{A \Delta T_{\text {Ar }}}=\frac{457 \mathrm{~W}}{\pi(.015 \mathrm{~m})(60.4 \mathrm{~m})(10 \mathrm{~K})}=16 \mathrm{~W} / \mathrm{m}^{2} \mathrm{~K}
\end{aligned}
$$

CHeck convection coefFicient obtainable.

$$
\begin{aligned}
& N_{u_{0}}=0.023 \operatorname{Re}_{0}{ }^{1 / 5} P_{r} \cdot 3 \\
& R e_{0}=22,700 \frac{q p}{d \mu} \quad q=8.8 \frac{g}{5} \times \frac{1}{.07118 \frac{\mathrm{mm}}{\mathrm{ft}^{3}}} \times \frac{2.216 \mathrm{~m}}{1000 \mathrm{~g}}=0.27 \mathrm{ft}^{3} / \mathrm{s} \\
& \rho=1.14 \mathrm{~kg} / \mathrm{m}^{3} \times(.06248)=.07118^{1 \mathrm{~mm} / \mathrm{ft}^{3}} \quad \mu=8.5 \mu \mathrm{Pa} \cdot \mathrm{s}=.0085 \mathrm{cp}
\end{aligned}
$$

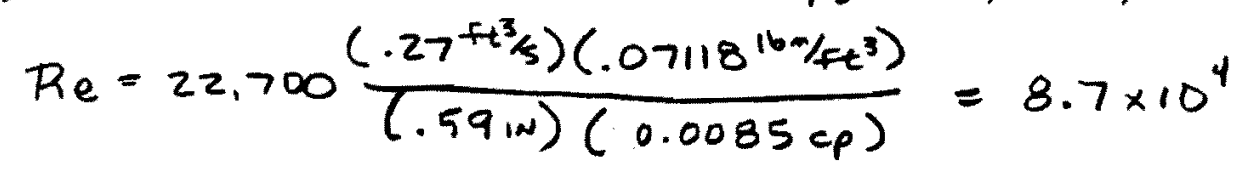

$$
\begin{aligned}
& \operatorname{Pr}=.68
\end{aligned}
$$




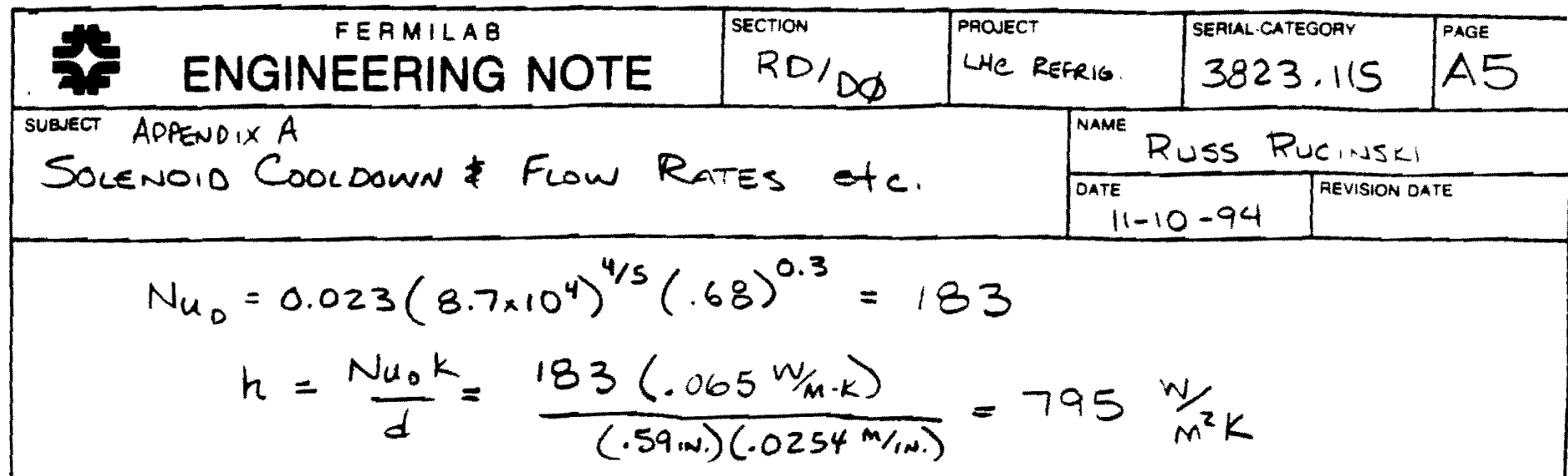

I conclude that fir step 5 we have plenty of heat transfer area and convection coefficient for

a $2.5 \mathrm{~K} / \mathrm{HR}$ CODLOOWN RATE.

$\rightarrow$ WE NEED tO SIZE COOLDOWN LINES For ACCEPTABLE $\triangle P$ WITH $\dot{m}=79 / \mathrm{s}, T=200 \mathrm{~K} \mathrm{~N}, 300 \mathrm{~K}$ ouT [STEP 1]

AND

$$
\dot{m}=99^{5}, \quad T=80 \mathrm{~K} \mathrm{in,} 100 \mathrm{~K} \text { OUT [STEP S] }
$$

- determining which $\triangle P$ will be largest;

$$
\begin{aligned}
& \Delta P \propto \frac{F L W^{2}}{\rho d s} \quad \frac{\Delta P_{S T S P_{2}}}{\Delta P_{\text {STEP }}}=\frac{f_{1} W_{1}^{2} \cdot \rho_{G}}{F_{4} W_{4}^{2} \rho_{1}} \\
& F_{1}=.03 \quad F_{5}=0.018 \text { From Moody Diadem } \\
& \rho_{5}=.07118^{1 \mathrm{lmm} / \mathrm{tt}^{3}} \quad \rho_{1}=.023^{\mathrm{lbm} / \mathrm{ft}^{3}}
\end{aligned}
$$

SUBSTITUTing;

$$
\frac{\Delta P_{\text {STEP 1 }}}{\Delta P_{\text {STEP F }}}=\frac{(.03)(7)^{2}(.07118)}{(.018)(9)^{2}(.028)}=2.6 \quad \therefore \underset{\substack{\text { STEP } 1 \text { NL BS } \\ \text { GREATER }}}{\Delta P}
$$

ConcLUsion:

size coOLdOWN lines for an acceptable $\triangle P$ WITH

$$
\dot{m}=7^{9 / 5}, T=200 \mathrm{~K} \text { in, } T=300 \mathrm{~K} \text { out }
$$




\section{SFECIFIC HEAT, ENTEALPY OF ALURINUM}

Sources of Data:

G1suque, W. F. and Mesds, P. F., J. Am. Chem. Soc. 63, 1897-1901 (1941)

Maler, C. G. and Anderson, C. T., J. Chem. Phys. 2, 513-27 (1934)

Ph1111ps, IN. E., Lov Temperature Phys1cs and Chentotry, Univ. W1sconsin Press (1958)

\section{Other References:}

Behn, U., Ann. Physik Belblitter 25, 178 (1901)

Goodmun, B. B., Compt. rend. $244, \frac{25}{2} 89$ (1957)

Griffiths, E. G. and Griffiths, E., Ph11. Trans. Roy. Soc. London A9O, 557 (1914)

Kok, J. A. and Keesom, W. H. , Physica 4, 835 (1937)

Koref, F., Ann. Physik (4) 36, 49 (1917)

Kernst, H., Ann. Physik (4) 36, 395 (1911)

Iermst, W. and Lindemann, F. A., Z. Elektrochem. 17, 817 (1911)

Mernst, W. and Schwera, F., S1tzber. kgl. preuse. Akad. W18s. 355 (1914)

Ruchards, T. W. and Jackson, F. G., Z. phys1k. Chem. 70, 414 (1910)

Schm1tz, в. E., Proc. Roy. Soc. (London) 72, 177 (1903)

T1lden, W. A., Proc. Roy. Soc. (London) $\underline{T}, 220$ (1903)

Table of Selected Values

\begin{tabular}{|c|c|c|c|c|c|}
\hline $\begin{array}{c}\text { Temp. } \\
{ }^{\circ} \mathrm{K}\end{array}$ & $\frac{C_{p}}{J / g^{m}-{ }^{\circ} K}$ & $\begin{array}{c}\mathrm{B} \\
\mathrm{j} / \mathrm{gm}\end{array}$ & $\begin{array}{c}\text { Temp. } \\
{ }^{\circ} \mathrm{K}\end{array}$ & $\mathrm{c}_{\mathrm{g}}^{\mathrm{C}_{\mathrm{m}}-{ }^{-} \mathrm{x}}$ & $\begin{array}{c}\mathrm{B} \\
\mathrm{J} / \mathrm{gm}\end{array}$ \\
\hline $\begin{array}{l}1 \\
1 \\
2 \\
3\end{array}$ & $\begin{array}{r}0.00010 * \\
.000051 \\
.000108 \\
.000176\end{array}$ & $\begin{array}{r}0.000025 \\
.000105 \\
.000246\end{array}$ & $\begin{array}{l}60 \\
70 \\
80 \\
90\end{array}$ & $\begin{array}{r}0.214 \\
.287 \\
.357 \\
.422\end{array}$ & $\begin{array}{r}3.64 \\
6.15 \\
9.37 \\
13.25\end{array}$ \\
\hline $\begin{array}{r}4 \\
6 \\
8 \\
10\end{array}$ & $\begin{array}{l}.000261 \\
.00050 \\
.00088 \\
.0014\end{array}$ & $\begin{array}{l}.000463 \\
.00121 \\
.0026 \\
.0049\end{array}$ & $\begin{array}{l}100 \\
120 \\
140 \\
160\end{array}$ & $\begin{array}{l}.481 \\
.580 \\
.654 \\
.713\end{array}$ & $\begin{array}{l}17.76 \\
28.4 \\
40.7 \\
54.4\end{array}$ \\
\hline $\begin{array}{l}15 \\
20 \\
25 \\
30\end{array}$ & $\begin{array}{l}.0040 \\
.0089 \\
.0175 \\
.0315\end{array}$ & $\begin{array}{l}.018 \\
.048 \\
.112 \\
.232\end{array}$ & $\begin{array}{l}180 \\
200 \\
220 \\
240\end{array}$ & $\begin{array}{l}.760 \\
.797 \\
.826 \\
.849\end{array}$ & $\begin{array}{r}69.2 \\
84.8 \\
101.0 \\
117.8\end{array}$ \\
\hline $\begin{array}{l}35 \\
40 \\
50\end{array}$ & $\begin{array}{l}.0515 \\
.0775 \\
.142\end{array}$ & $\begin{array}{r}.436 \\
.755 \\
1.85\end{array}$ & $\begin{array}{l}260 \\
280 \\
300\end{array}$ & $\begin{array}{l}.869 \\
.886 \\
.902\end{array}$ & $\begin{array}{l}135.0 \\
152.5 \\
170.4\end{array}$ \\
\hline
\end{tabular}

* Superconducting

Reprinted from WADD TECH. REPORT 60-56 


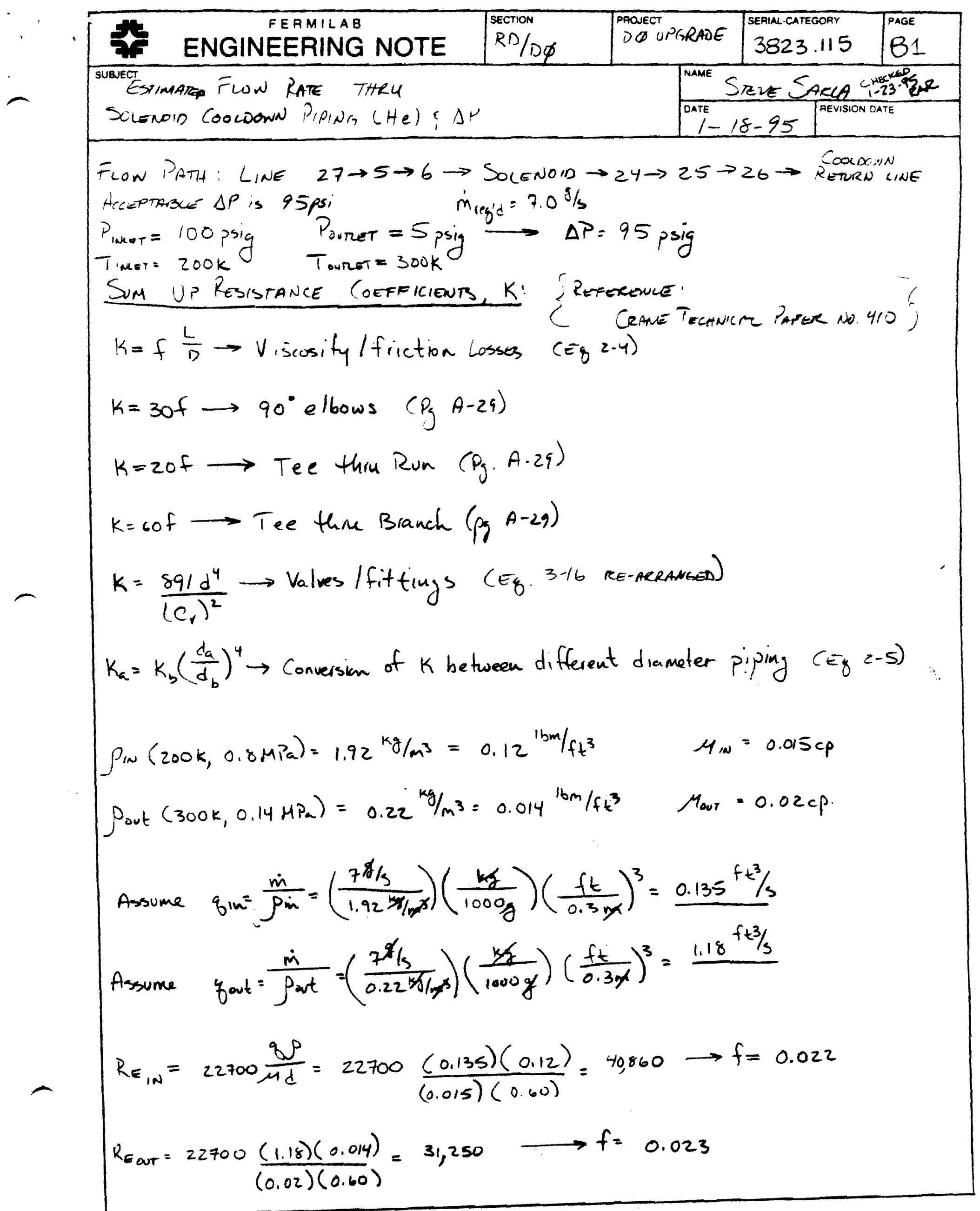




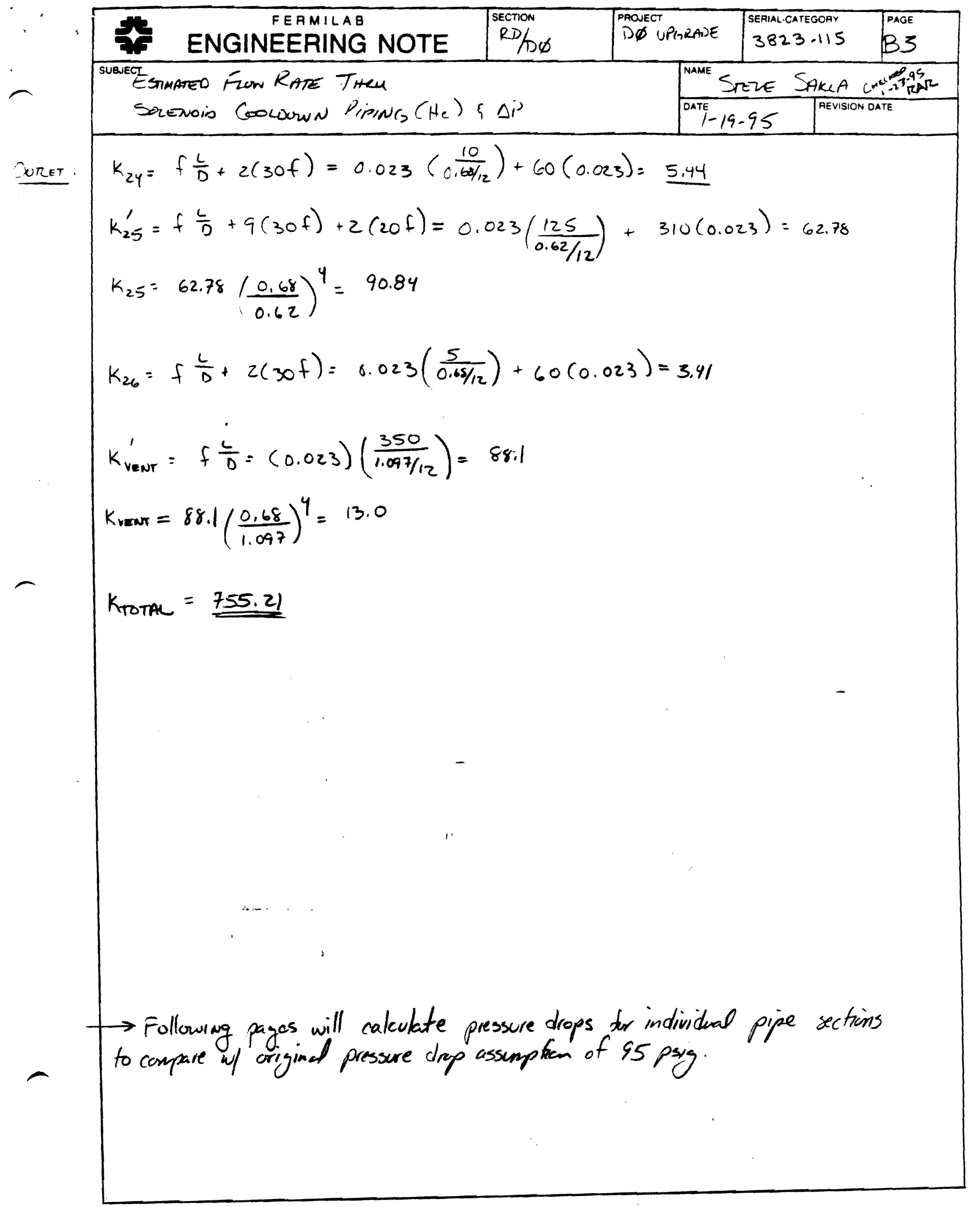




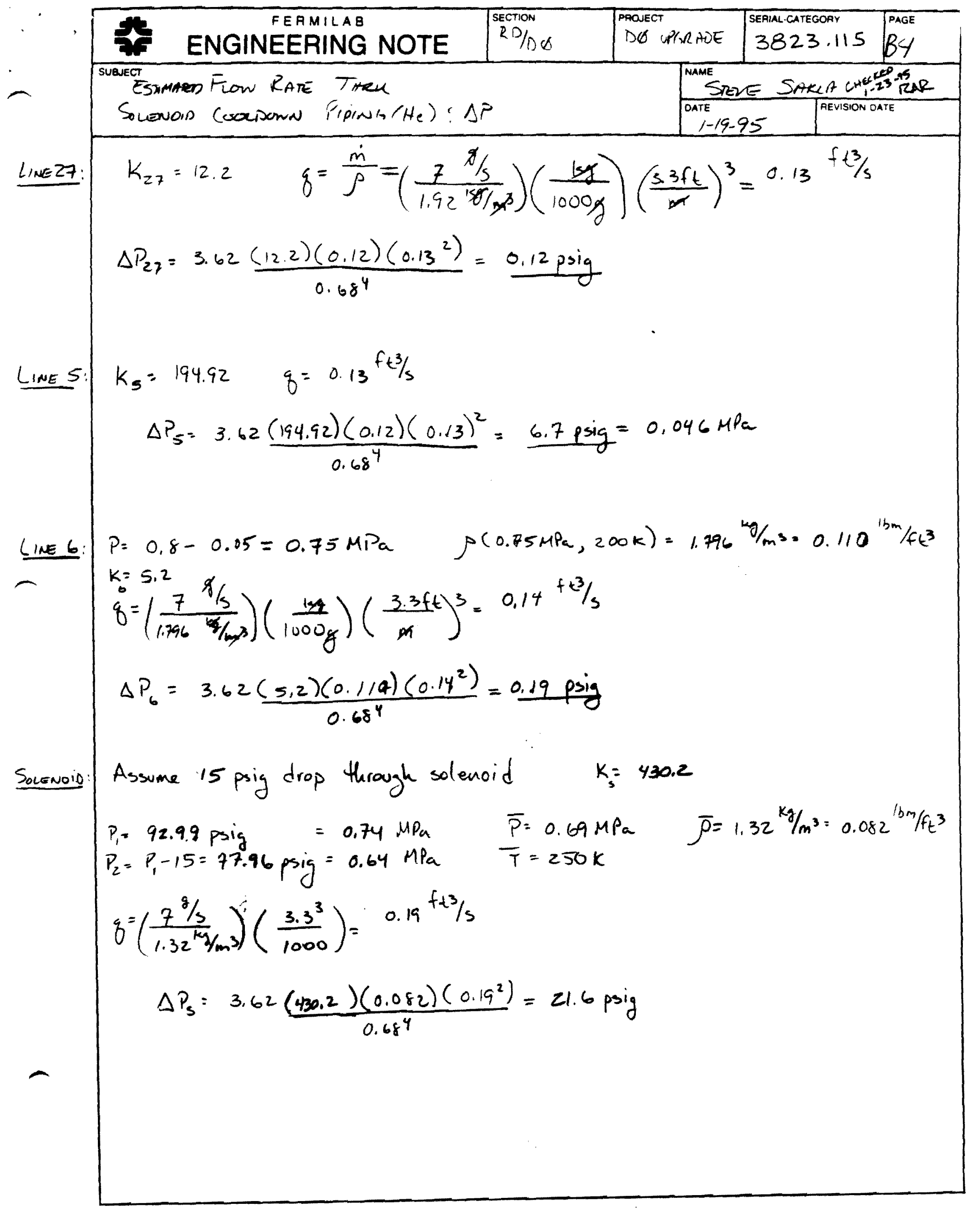




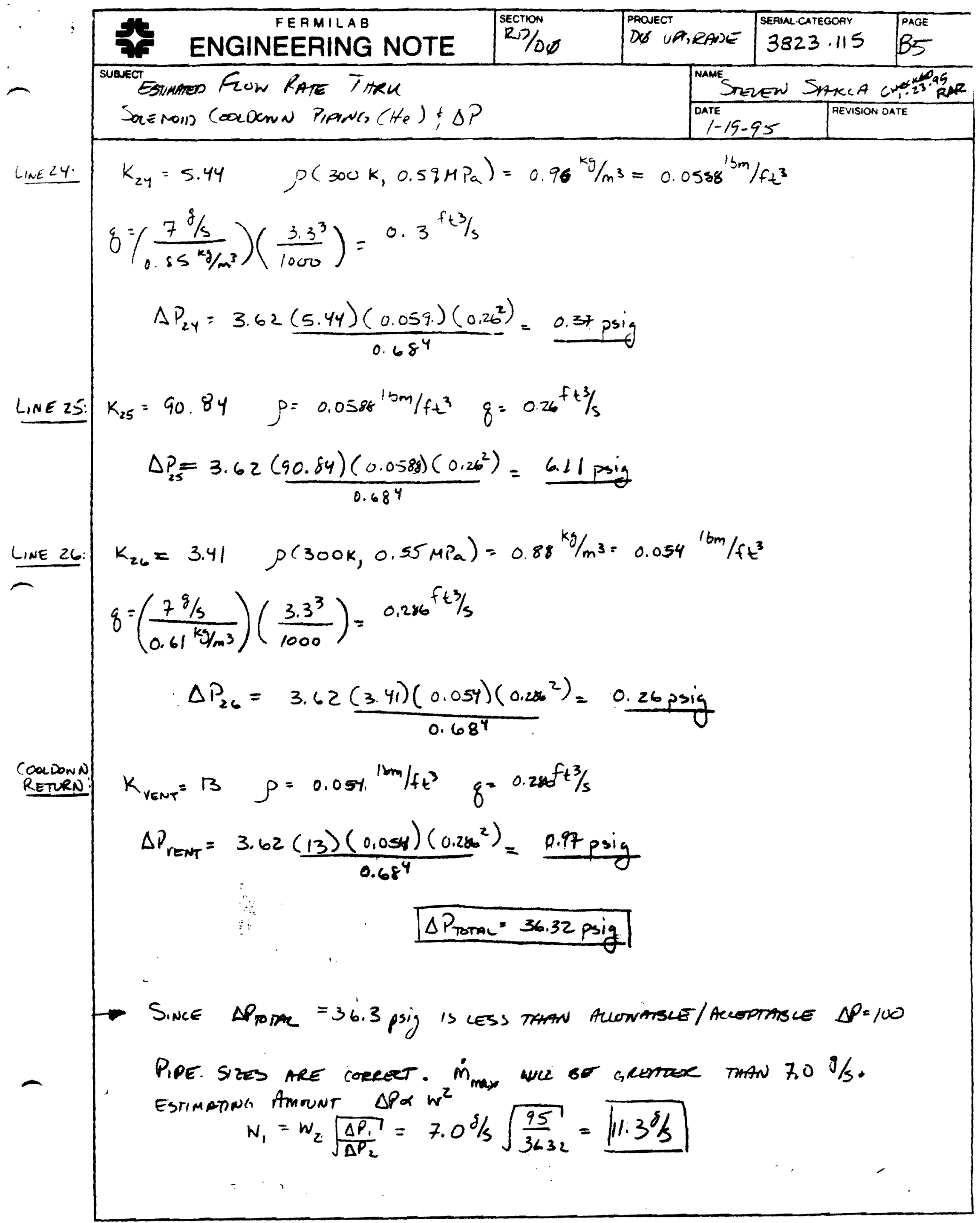




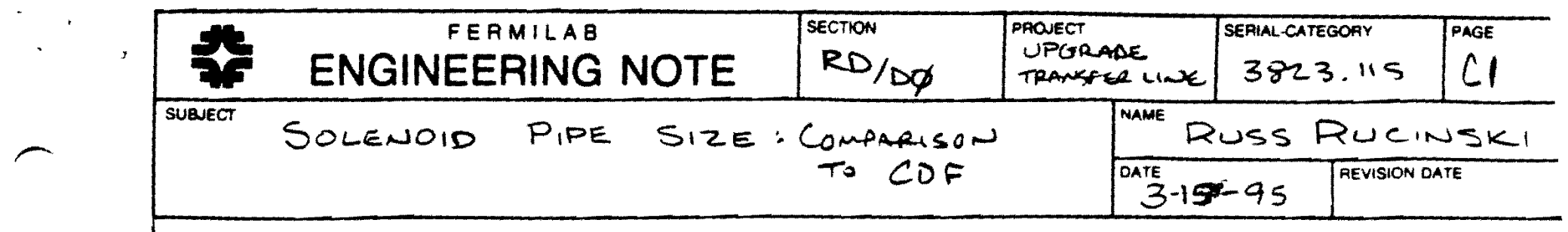

It can be assumed that cooldown process also DROVE THE PDF SOLENOID PIPE SIZING. IT IS USEFUL TO TAKE A COMPARABLE LOOK AT THE SIZES USED FOR CDF AND THOSE CHOSEN FOR D $\varnothing$.

THE ACTUAL COOLOOWN FLONTRATES ARE DRIVEN by thermal colo mass.

$$
\begin{aligned}
& \text { DD MAGNET COD MASS }=1460 \mathrm{~kg} \text { REG. DESIW RGIORT } \\
& \text { Taste A.1 Tm-1886 } \\
& \text { CF } \\
& =5568 \mathrm{~kg} \\
& \text { RAF. OSF Desiw RePort } \\
& M_{D D / M_{C D C}}=1460 / 5568=\frac{1 / 4}{3}
\end{aligned}
$$

From Crane TECHNICAM PATER 910

$$
\Delta p \propto \frac{f L w^{2}}{\rho d^{5}}
$$

Comparing the two systems a assuming L jp, f approx.

the same;

$$
d_{D \phi}^{S}=d_{C D P}^{5}\left[\left(\frac{W_{D D}}{W_{C O F}}\right)^{2}\left(\frac{\Delta P_{C D F}}{\Delta P_{D D}}\right)\right]
$$

We can sale that the acceptable $\triangle P$ for CDF $D \Phi$ are THe SAME.

$$
\triangle P_{C P F} / \Delta P_{D} \approx 1.0
$$

THE MASS FLOW RATE FOR DD WII BE $1 / 4$ LESS THAN ThAT READ FOR CDF SINCE IT HAS 1/4 Mrs. Substiatirte

$$
\begin{gathered}
d_{D \phi}^{5}=d_{C D P}^{5}\left[\left[\frac{1}{4}\right]^{2}(1)\right] \\
d_{D D}=(.33) d_{C D F}
\end{gathered}
$$




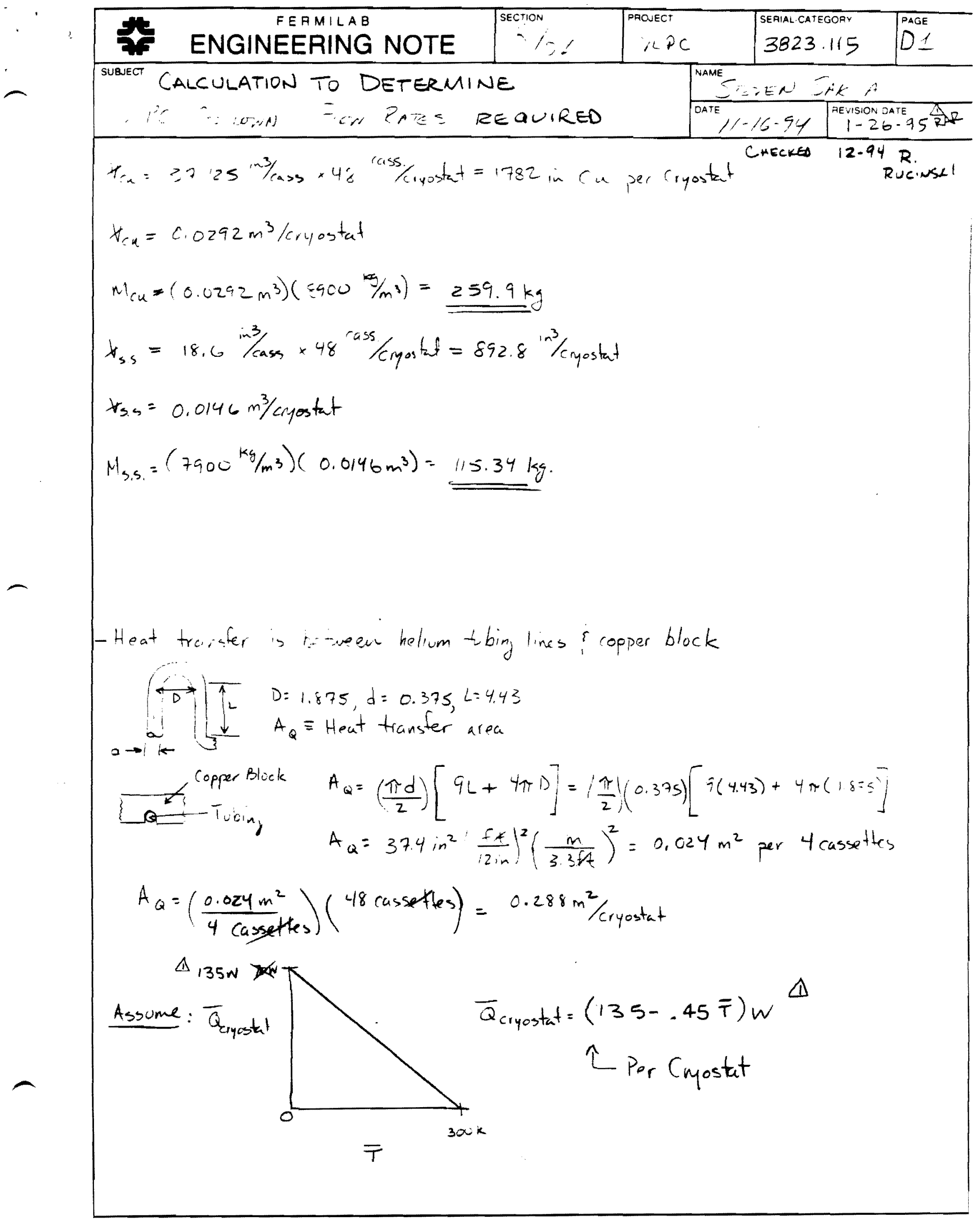




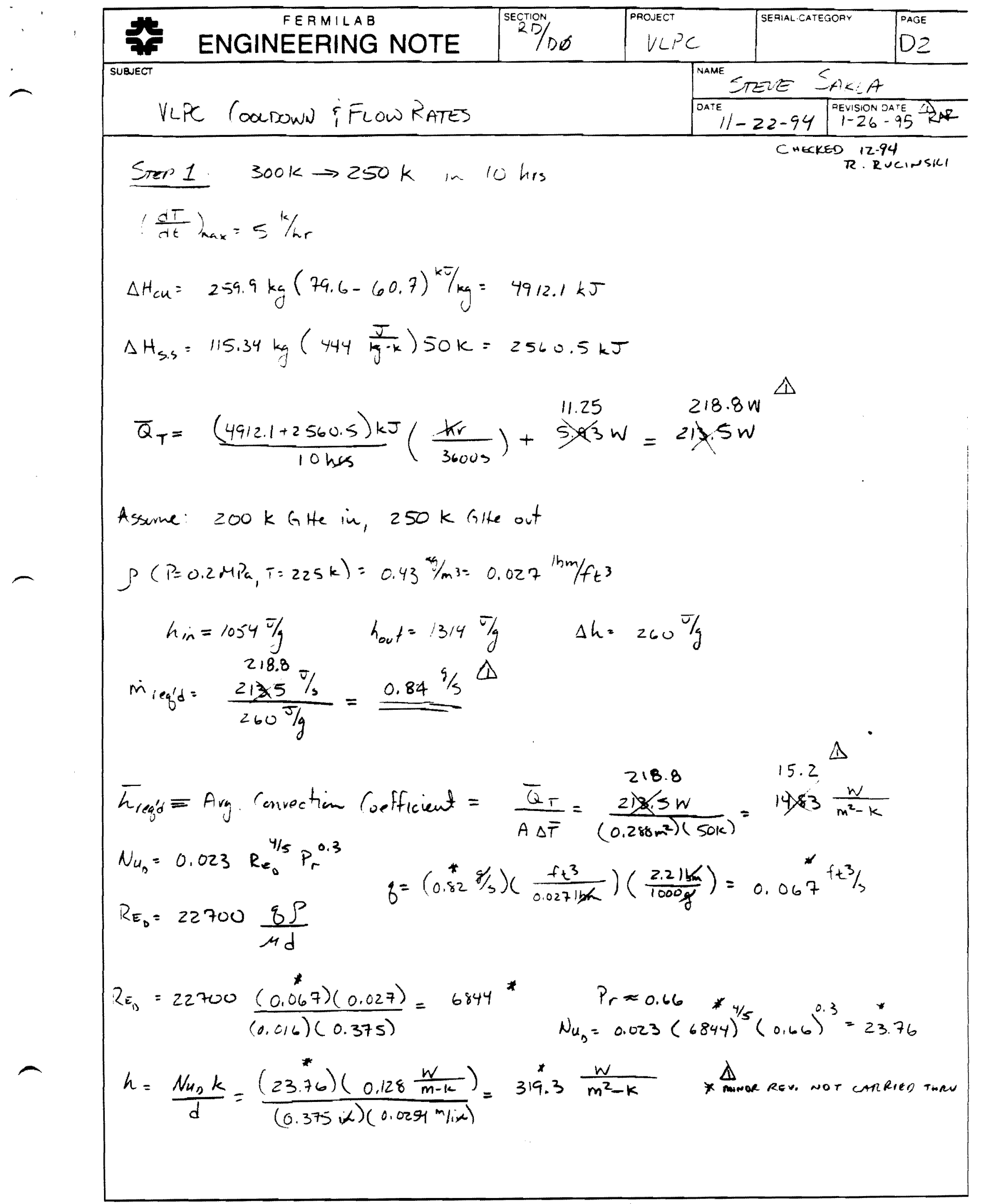




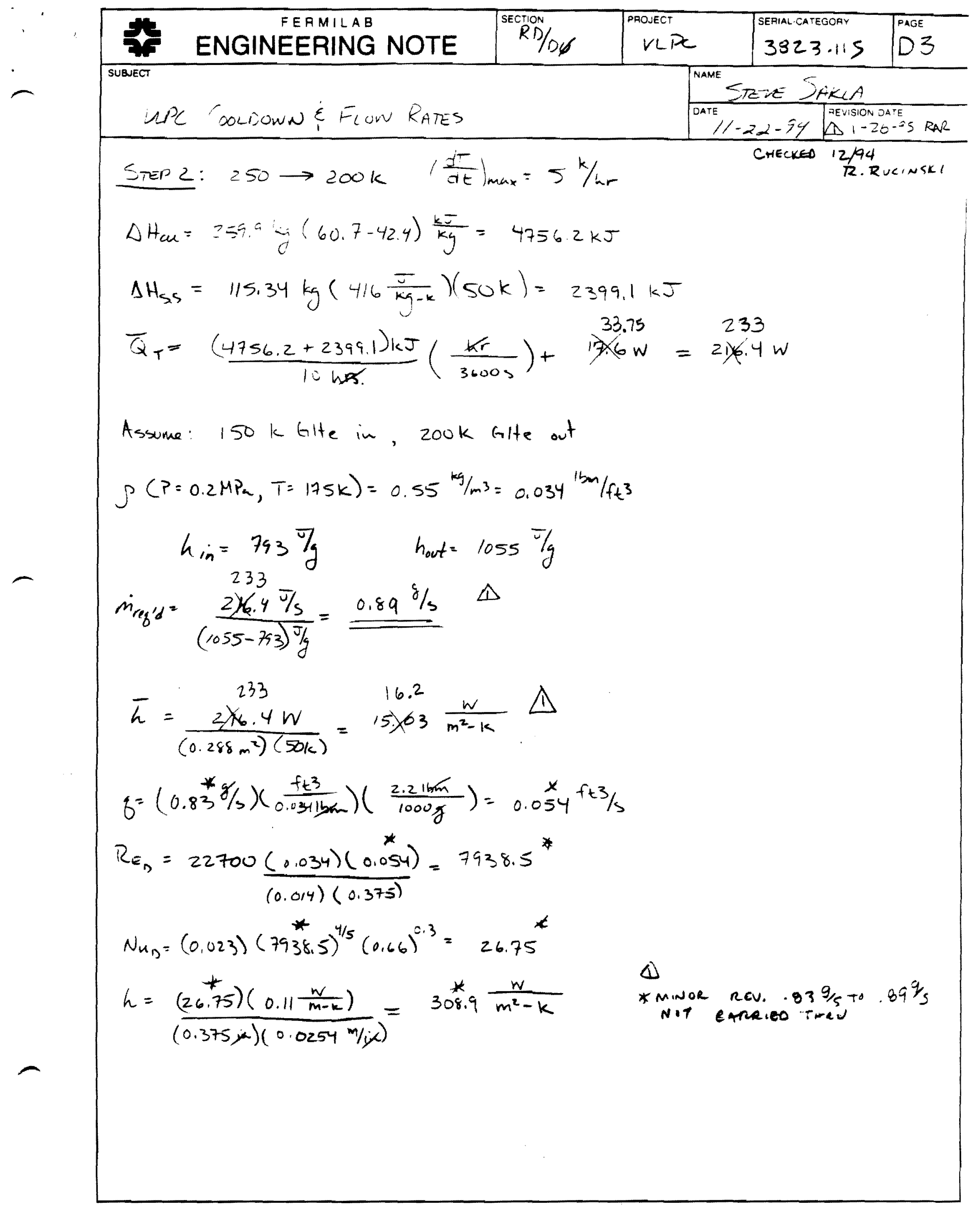




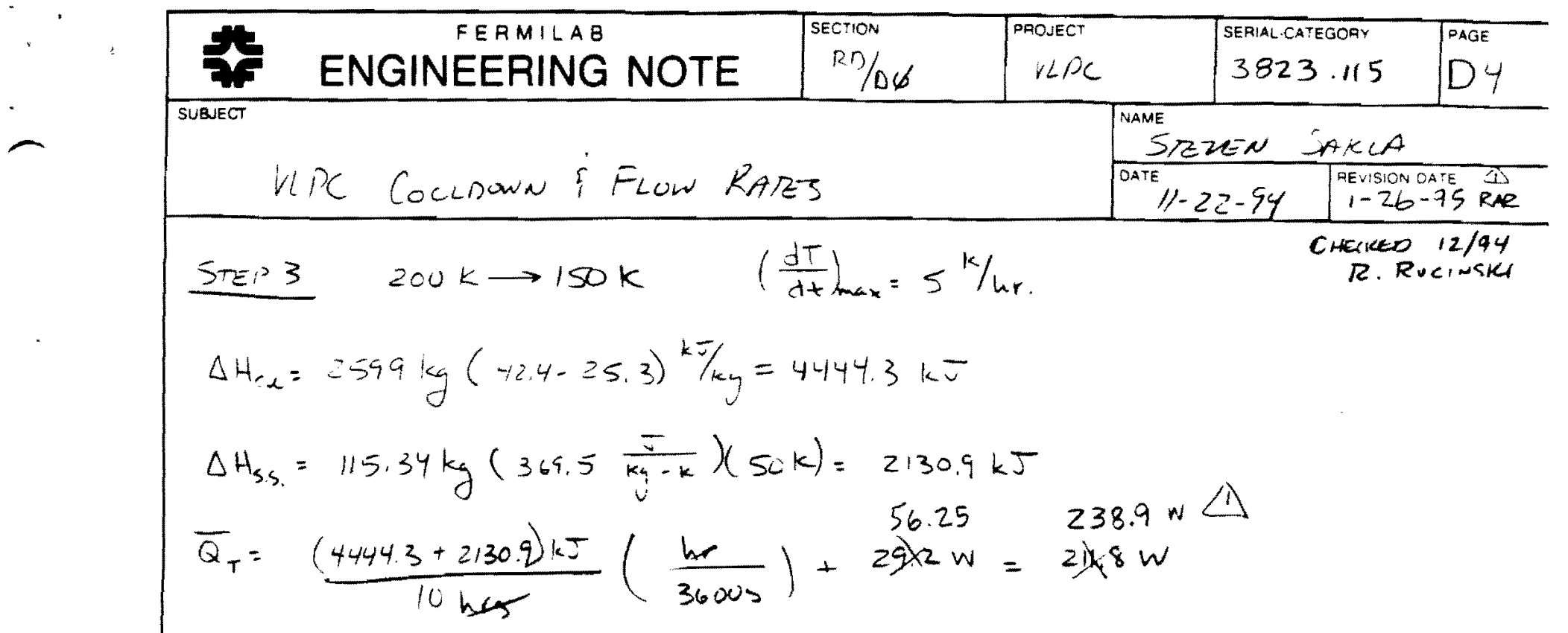

Assime $100 \mathrm{k}$ GHe in, $150 \mathrm{k}$ Gite out

$$
\begin{aligned}
& \partial(T=125 \mathrm{k})=0.77^{\mathrm{kg}} / \mathrm{m}^{3}=0.048^{16 \mathrm{~m}} / \mathrm{ft}^{3} \\
& h_{\text {in }}=535.1 \mathrm{~T} / g \quad h_{\text {out }}=793 \mathrm{~J} / \mathrm{g} \\
& \dot{m}_{\text {reqid }}=\frac{238.9 \mathrm{~J} / \mathrm{s}}{(793-535.1) \mathrm{F} / \mathrm{g}}=0.92 \mathrm{~g} / \mathrm{s} \\
& \bar{h}_{\text {reqld }}=\frac{238.9 \mathrm{~W}}{\left(0.288 \mathrm{~m}^{2}\right)(50 \mathrm{k})}=14 \times 71 \frac{\mathrm{w}}{\mathrm{m}^{2}-\mathrm{k}} \\
& q=(0.82 \% / \mathrm{s})\left(\frac{f_{t^{3}}}{0.6481 \% \mathrm{~m}}\right)\left(\frac{2.216 \mathrm{~m}}{1000 \mathrm{~g}}\right)=0.038 \mathrm{fz} 3 / \mathrm{s} \\
& R E_{b}=22700 \frac{(0.038)(0.048)}{(0.011)(0.375)}=10038 \\
& N_{u_{D}}=(0.023)(10038)^{* 4 / 5}(0.66)^{0.3}=32.3^{*} \\
& h=\frac{(32.3)\left(0.086 \frac{w}{m-k}\right)}{(0.375 .4)(0.0254 \mathrm{~m} / \mathrm{k})}=291.6^{*} \frac{w}{\mathrm{~m}^{2}-k}
\end{aligned}
$$




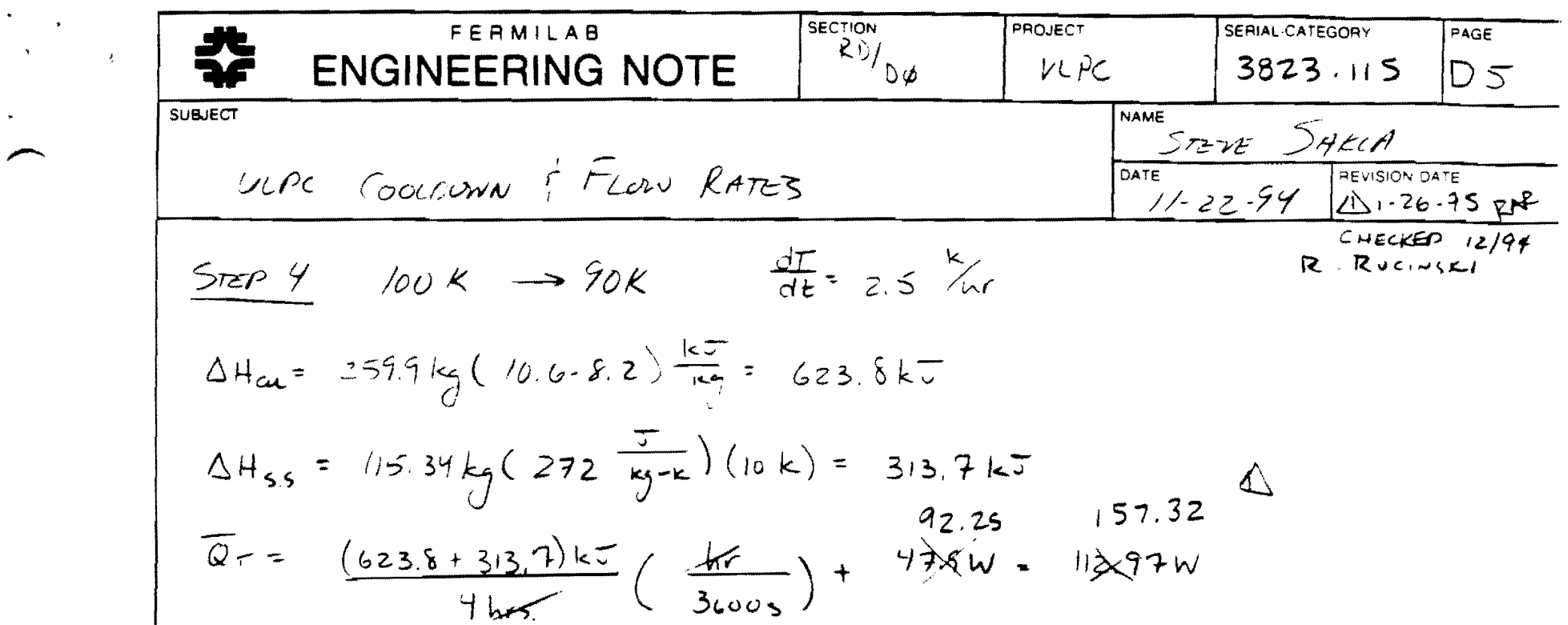

Assume sok Glte in, gck Glte out

$$
\begin{aligned}
& h_{\text {in }}=431.2 \mathrm{~J} / \mathrm{g} \quad h_{\text {out }}=483.2 \mathrm{~J} / \mathrm{g} \quad \rho(T=85 \mathrm{~K})=1.14 \mathrm{~kg} / \mathrm{m}^{3}=0.071^{16 \mathrm{~mm} / \mathrm{ft}^{3}} \\
& \begin{array}{l}
\dot{m}_{\text {reqd }}=\frac{112 \times 97 \mathrm{~J} / \mathrm{s}}{(483.2-431.2) \mathrm{J} / \mathrm{g}}=3 \times 2 \mathrm{~g} / \mathrm{s} \\
\bar{h}_{\text {req'd }}=\frac{112.57 \mathrm{~W}}{\left(0.288 \mathrm{~m}^{2}\right)(10 \mathrm{k})}=31.2 \frac{\mathrm{w}}{\mathrm{m}^{2} \cdot \mathrm{k}}
\end{array} \\
& q=(2.2 \% / \mathrm{s})\left(\frac{\mathrm{ft} 3}{0.0711 \mathrm{sm}}\right)\left(\frac{2.21 \mathrm{hm}}{1000 \mathrm{~J}}\right)=0.096 \mathrm{ft}^{3} / \mathrm{s} \\
& R_{E_{0}}=22700 \frac{(.096}{(0.004)(0.375)}=3249345,757 \\
& N_{u_{0}}=(0.023)(33 \times 173)^{4 / 5}(0.66)^{0.3}=82.6108 .6 \\
& h=\frac{(88.6)\left(0.066 \frac{w}{m-k}\right)}{(0.375 .67)(0.0254 \mathrm{~m} / \mathrm{kL})}=572.3 \frac{w}{\mathrm{~m}^{2}-\mathrm{k}}
\end{aligned}
$$


SPECIFIC EEAT, ENTHALPY OF COPFER

$$
\left(10^{\circ} \text { to } 300^{\circ} \mathrm{K}\right)
$$

\section{Sources of Drta:}

Doviersy, S. M., Cen. J. Research 15A, 59-6́́ (1937)

\section{Ot I Feferences:}

Ac:a-e, S. snd Karia, E., J. Chem. Soc. Jepan 62, 312-15 (1941)

Ee:a, U., inn. Pajsin u. Chem. (3) 66, 237-44 (1ミ98)

E-crson, B. L., Caisholm, I. M. and Doc'erty, S. M., Can. J. Sesearch 8, 292-303 (1933)

Zuiven, A. and ierth, I., Z. anorg. allgem. Coer. 188, Schenck Festscis: $=t, 152-12(2930)$

Gingue, W. F. and Hesds, P. F., J. Am. Chem. Sos. б́3, 1897-2901 (1941)

Keesod, W. H. and Onnes, M. K., Communs. Fays. Lai. Undr. Leldea No. $24 i a, 3$ (1915)

korer, F., Ano. Faysix 36, 49-73 (1911)

lie:nst, W., s1tzber. sgl. preuss. Aj̈ad. Wiss. 262 (1910)

Beinst, W., S1tziber, kgl. preuss. Akad. Wiss. 306 (1911)

Vierst, W. and LInderann, F. A., Z. Elektrochem. 17, 817 (1911)

Sch1mpif, \#., Z. phys1k. Chem. 71, 257 (1910)

Table or selected Values

\begin{tabular}{|c|c|c|c|c|c|}
\hline $\begin{array}{c}\text { Teup. } \\
x\end{array}$ & $\overbrace{i=-{ }^{\circ} K}^{C_{p}}$ & $\begin{array}{c}E^{*} \\
j / \mathbb{B}^{\circ}\end{array}$ & $\begin{array}{c}\text { Temp. } \\
x\end{array}$ & $c_{p}^{C_{p}}$ & $\begin{array}{r}\text { B" } \\
\mathrm{J} / \mathrm{Sm}\end{array}$ \\
\hline $\begin{array}{l}10 \\
15 \\
20 \\
25\end{array}$ & $\begin{array}{c}0.00086 \\
.0027 \\
.0077 \\
.016\end{array}$ & $\begin{array}{c}0.0024 \\
.0107 \\
.034 \\
.090\end{array}$ & $\begin{array}{l}100 \\
120 \\
140 \\
160\end{array}$ & $\begin{array}{r}0.254 \\
.288 \\
.313 \\
.332\end{array}$ & $\begin{array}{l}10.6 \\
16.1 \\
22.1 \\
28.5\end{array}$ \\
\hline $\begin{array}{l}30 \\
40 \\
50 \\
60\end{array}$ & $\begin{array}{l}.027 \\
.060 \\
.099 \\
.137\end{array}$ & $\begin{array}{l}.195 \\
.61 \\
1.40 \\
2.58\end{array}$ & $\begin{array}{l}180 \\
200 \\
220 \\
240\end{array}$ & $\begin{array}{l}.346 \\
.356 \\
.364 \\
.371\end{array}$ & $\begin{array}{l}35.3 \\
42.4 \\
49.6 \\
56.9\end{array}$ \\
\hline $\begin{array}{l}70 \\
80 \\
90\end{array}$ & $\begin{array}{l}.173 \\
.205 \\
.232\end{array}$ & $\begin{array}{l}4.13 \\
6.02 \\
8.22\end{array}$ & $\begin{array}{l}260 \\
280 \\
300\end{array}$ & $\begin{array}{l}.376 \\
.381 \\
.386\end{array}$ & $\begin{array}{l}64.4 \\
72.0 \\
79.6\end{array}$ \\
\hline
\end{tabular}

keprinted 


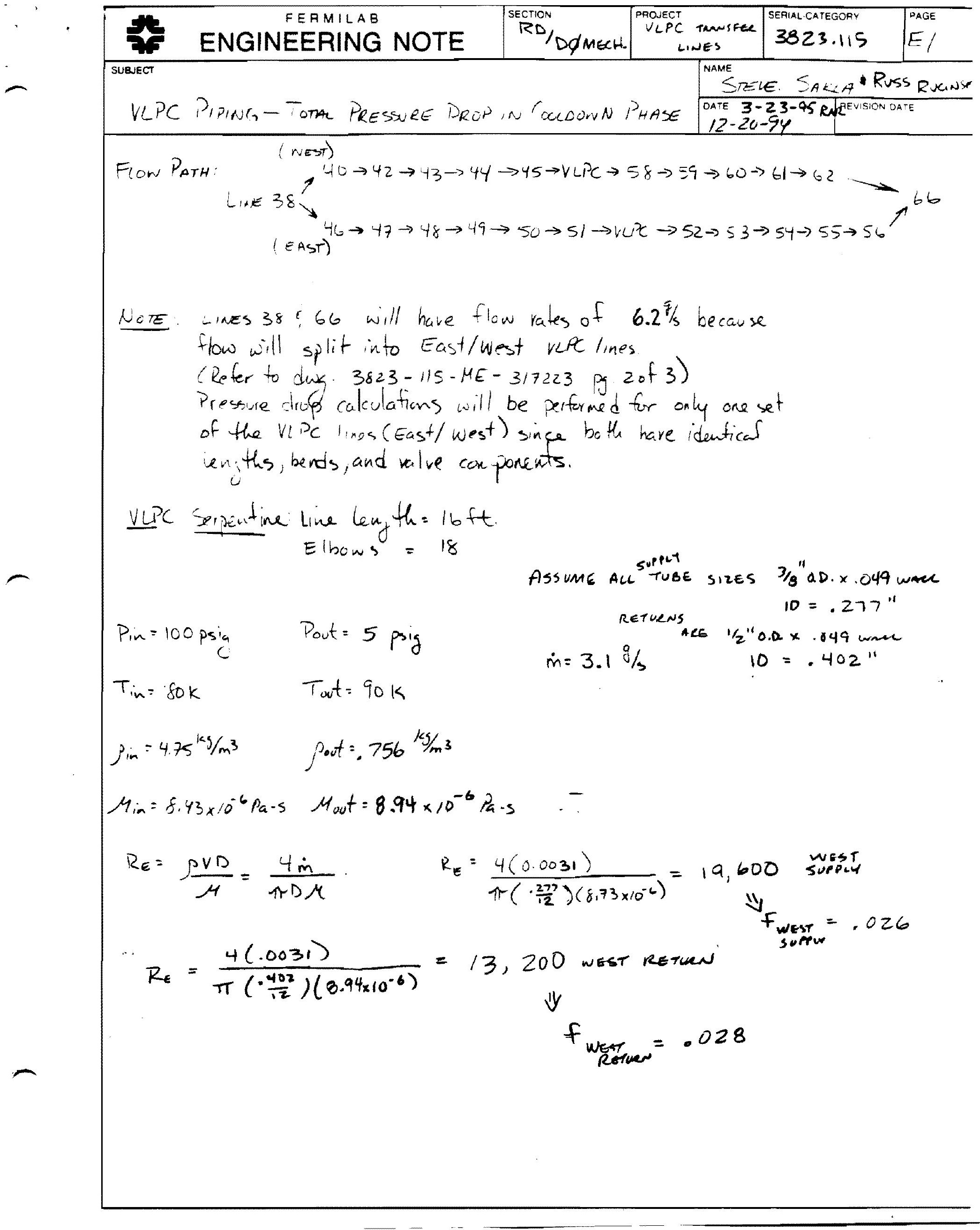




\section{$15 \quad$ raie \\ Armco}

(99.758, pure)

7870

447

7854

Carbon steels

(Mn $\leq 1 \%$,

Si $\leq 0.1 \%)$

Als1 1010

Carbon-silicon

(Mn $\leq 1 \%$,

$0.1 \%<\mathrm{Si} \leq 0.6 \%)$

Carbon-manganese-

silicon

$(1 \%<\mathrm{Mn} \leq 1.65 \%$.

$0.1 \%<\mathrm{Si} \leq 0.6 \%)$
$7817 \quad 446$

$8131 \quad 434$

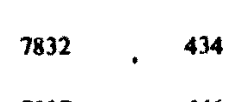

$+\mathrm{Cr}-1 \mathrm{Mo}-\mathrm{Si}$ (0.18\% C, $0.65 \% \mathrm{C}$

$0.23 \% \mathrm{Mo}, 0.6 \% \mathrm{Si}$ )

$1 \mathrm{Cr}-\frac{1}{2} \mathrm{Mo}$

(0.16\% C. 1\% Cr.

$0.54 \% \mathrm{Mo}, 0.39 \% \mathrm{Si}$ )

$1 \mathrm{Cr}-\mathrm{V}$

(0.2\% $\mathrm{C}, 1.02 \% \mathrm{Cr}$

$0.15 \%$ v)

Stainless steels

AISI 302

AISI 304

AISI 316

AISI 347

Lead

Magnesium

Molybdenum

Nickel

Pure

Nichrome

( $80 \% \mathrm{Ni}, 20 \% \mathrm{Cr}$ )

Inconel X-750

(73\% $\mathrm{Ni}_{1}$ 15\% $\mathrm{Cr}$.

$6.78 \mathrm{Fe}$ )

Niobium

Palladium

Platinum

Pure

$\begin{array}{llllllll}216 & 384 & 490 & 574 & 680 & 975 & 609 & 654\end{array}$

$\begin{array}{llllllll}95.6 & 80.6 & 65.7 & 53.1 & 42.2 & 32.3 & 28.7 & 31.4\end{array}$

$\begin{array}{llllllll}215 & 384 & 490 & 574 & 680 & 975 & 609 & 654\end{array}$

$60.5 \quad 17.7$

$\begin{array}{rrrr}56.7 & 48.0 & 39.2 & 30.0 \\ 487 & 559 & 685 & 1169\end{array}$

$\begin{array}{llll}58.7 & 48.8 & 39.2 & 313\end{array}$

$\begin{array}{rrrr}58.7 & 46.8 & 39.2 & 31.3 \\ 487 & 559 & 685 & 1168\end{array}$

$\begin{array}{lllll}49.8 & 440 & 37.4 & 29.3\end{array}$

$\begin{array}{llll}501 & 582 & 699 & 971\end{array}$

$\begin{array}{llll}42.2 & 39.7 & 35.0 & 27.6\end{array}$

$\begin{array}{llll}487 & 559 & 685 & 1090\end{array}$ 


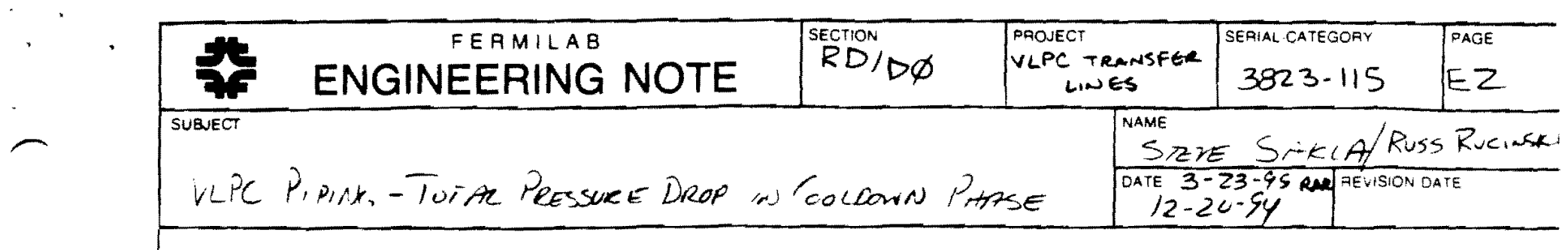

Sim in Resistance loffeirients, K.

$$
\begin{aligned}
& K=f \frac{L}{D} \longleftarrow \text { Viscosing/Friction } \quad k=60 f \leftarrow \text { Tee thru branch } \\
& k=30 \mathrm{f} \longleftarrow 00^{\circ}=1 \text { bows } \quad k=20 \mathrm{f} \longleftarrow \text { Tee thru Run } \\
& K=20 f \longleftarrow \text { Tee thru Run } \quad K=8 f \longleftarrow \text { Pressure Valve } \\
& k=\frac{891 d^{4}}{(C,)^{2}} \quad k=100 \mathrm{~F} \leftarrow \text { Check Value } \\
& \operatorname{Re}=27,000 \\
& 1 / 2 " x .049 \text { tube } \\
& 10=.402 " \\
& K_{\text {in }}^{\prime}=F \frac{L}{D}+2(30 F)=0.024\left(\frac{5}{. \frac{402}{12}}\right)+60(0.024)=5.02 \\
& K_{1 N}=5.02\left(\frac{-207}{.402}\right)^{4} \cdot-35
\end{aligned}
$$

WEST VLPC SUPPLY LINES: $40 \rightarrow 42 \rightarrow 43 \rightarrow 44 \rightarrow 45 \rightarrow$ VLPC

$$
\begin{gathered}
\text { Lome }=2+123+12+47+24+16=224 \\
f=0.026 \quad k=0.026\left(\frac{224}{\frac{.277}{12}}\right)=252 \\
\left(+90^{\circ} \text { elbows }\right)_{T}=1+9+2+6+2+18=38 \\
K_{e}=(38)(30)(0.026)=\underline{29.6} \\
\text { \#Tees Thru } \left.R_{\text {Un }}\right)_{T}=1+3+1=5 \\
K_{\text {TR }}=(5)(20)(0.026)=2.6
\end{gathered}
$$

Valves: I diverter vale, $C_{V}=5.0$

$$
\begin{aligned}
& K_{v}=\frac{891(.277)^{4}}{5^{2}}=.0002 \rightarrow \text { NEQUIHBLE } \\
& K_{\text {SuPT }}=252+29.6+2.6=\frac{284}{7} \text { BASE O ON .207"10. }
\end{aligned}
$$




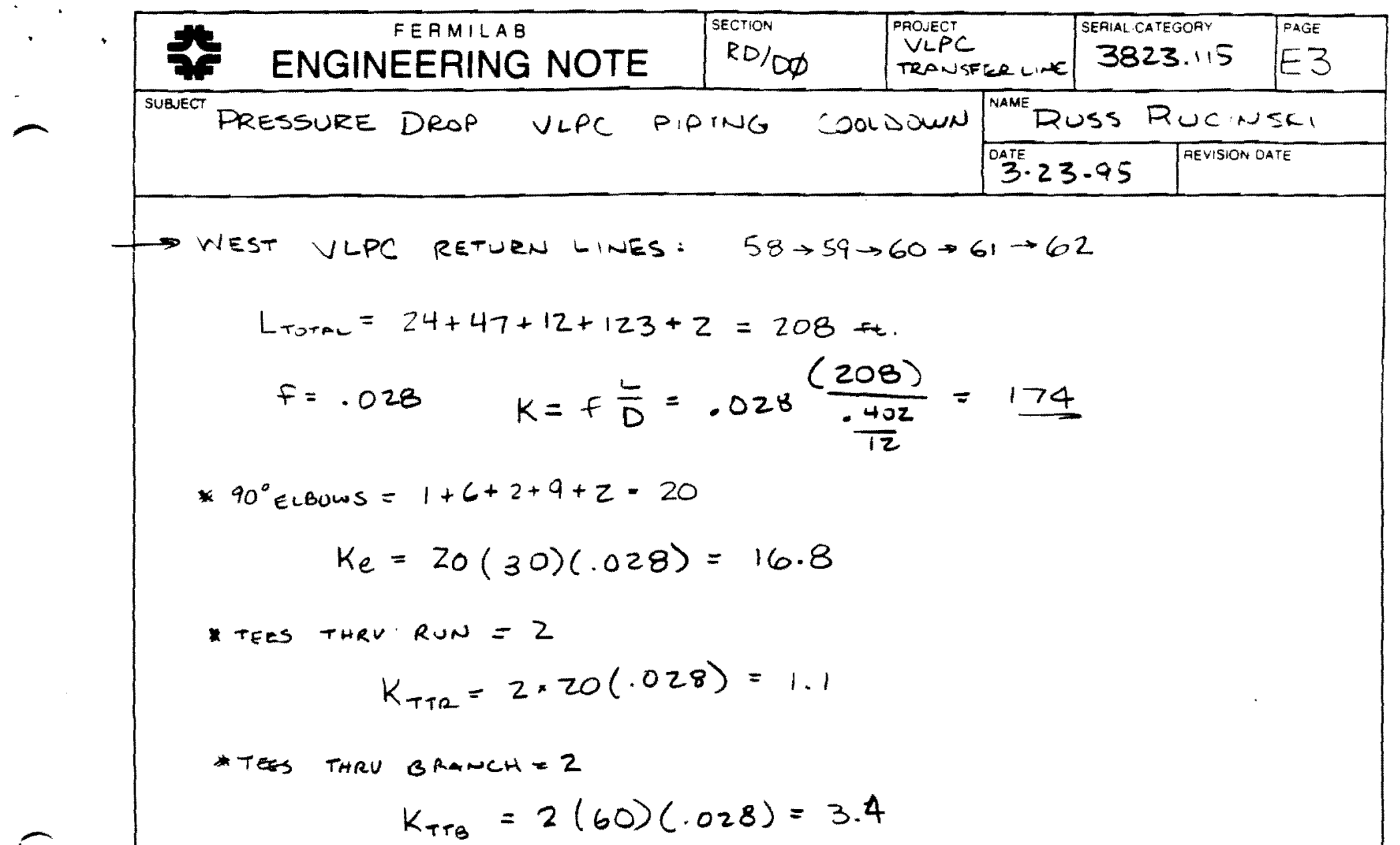

valves: 1 control value $C_{V}=2.0$, 1 diverter value $\Rightarrow C_{V}=5.0,1$ check value

$$
\begin{aligned}
& K=\frac{891(.402)^{4}}{(2.0)^{2}}+\frac{891(.402)^{4}}{(5.0)^{2}}+100(.028)=5.8+.9+2.8=9.5 \\
& K_{\text {RETURN }}=174+16.8+1.1+3.4+9.5=205 \text { BASE O ON. } 402 \times 10 .
\end{aligned}
$$

return line: $* 66$

$L=5 \mathrm{Ft} 2$ elbows, same supply $* 38$

$$
K_{\text {OUT }}=5.02 \text { BASED ON - } 402 " 10 \text {. }
$$

Pressure Drop

$$
\begin{aligned}
& q=\frac{\dot{m}}{\rho}=\left(\frac{6.2}{\mathrm{~s}} \mathrm{~g}\right)\left(\frac{\mathrm{kg}}{1000 \mathrm{~g}}\right)\left(\frac{2.2 \mathrm{~km}}{1 \mathrm{~kg}}\right)\left(\frac{+t^{3}}{.297 \mathrm{lom}}\right)=.04593^{\mathrm{ft} / \mathrm{s}} \\
& \Delta P_{38}=3.62 \frac{\mathrm{k} \rho g^{2}}{d^{4}}=3.62 \frac{(5.02)(.297)(.04593)^{2}}{(.402)^{4}}=0.436 \mathrm{psi}
\end{aligned}
$$




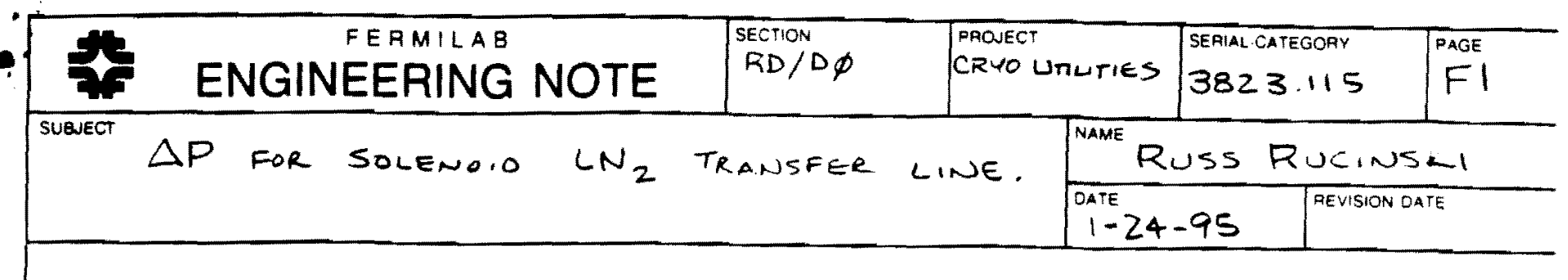

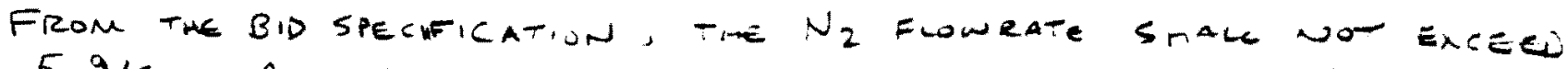
$5 \mathrm{9} / \mathrm{s}$. As a worst CASE I WILL calculate THE $\triangle P$ in the piping O $T_{\text {aug }}=300 \mathrm{~K}$. P arg. 30 psia.

THE PIPE sizes are abreast preininatruy sized.

Flow PATH

SUPPLY:

$$
\begin{array}{ll}
10 \rightarrow 11 \rightarrow 12 \rightarrow 13 & \text { (SHIELDS) } \\
10 \rightarrow 11 \rightarrow 14 \rightarrow 15 & \text { (INTERCEPT) }
\end{array}
$$

Calculations WILL ONLY BE DONE FOR THE SUPPG FROM THE LN Z HEADER TO THE SOLENOID. THE RETURN Flow Path is identical with regard to pipe size

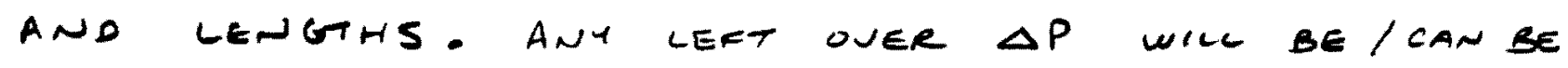
used at the control valves and in solano.

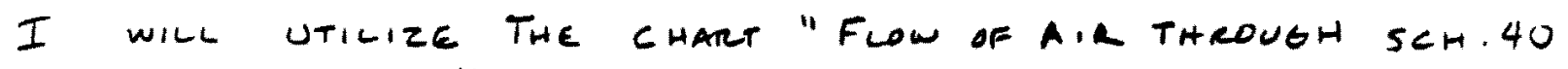
STEel PIPE" PG. B.15, crane technical paper 410.

$\triangle P$ PER 100 fE is GIVEN For JARIOUS FWU RATES \& PIPE sizes.

Converting $59 / 5$ to FreE AIR;

$$
\begin{aligned}
& q_{m}^{\prime} \approx 5 \frac{9}{\mathrm{~s}} \times 878.54 \cdot \frac{\mathrm{cm}^{3}}{2} \times \frac{60 \mathrm{~s}}{1 \mathrm{~min} .} \times\left[3.53 \times 10^{-5} \frac{\mathrm{ft}^{3}}{\mathrm{~cm}^{3}}\right] \\
& q_{m}^{\prime}=9.30 \mathrm{scfin} v(T=300 \mathrm{~kg}, \mathrm{P}=1 \mathrm{ATM})
\end{aligned}
$$

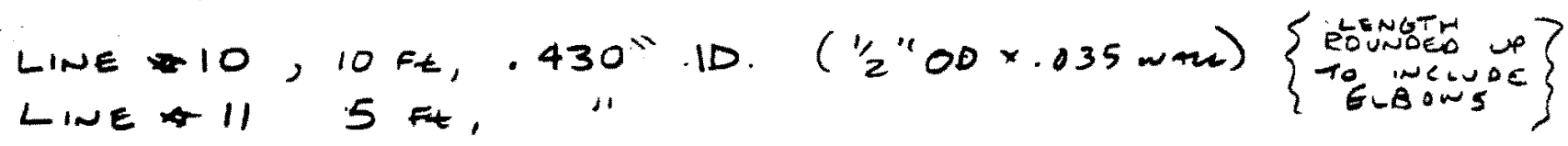

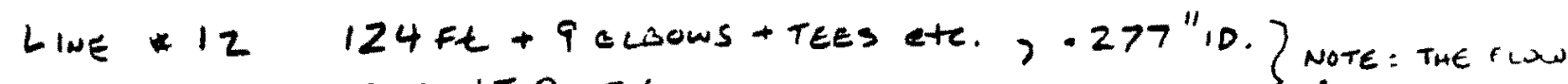
SAT $150 \mathrm{Ft}$.

LINE * $1310 \mathrm{Ft}, .430 \% 10$.

Rate rill BE $1 / 2$ HERE DUE TO SPLIT 


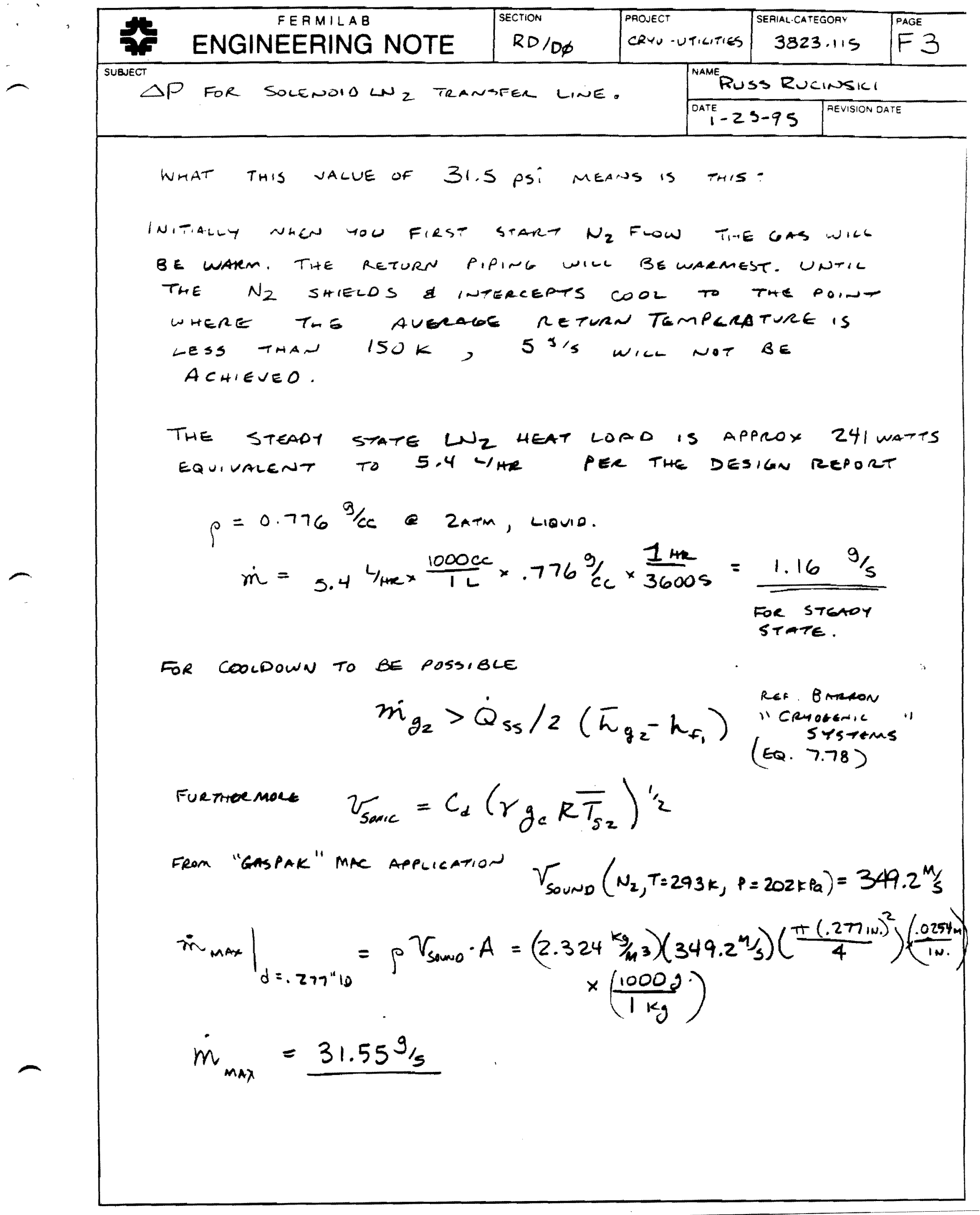




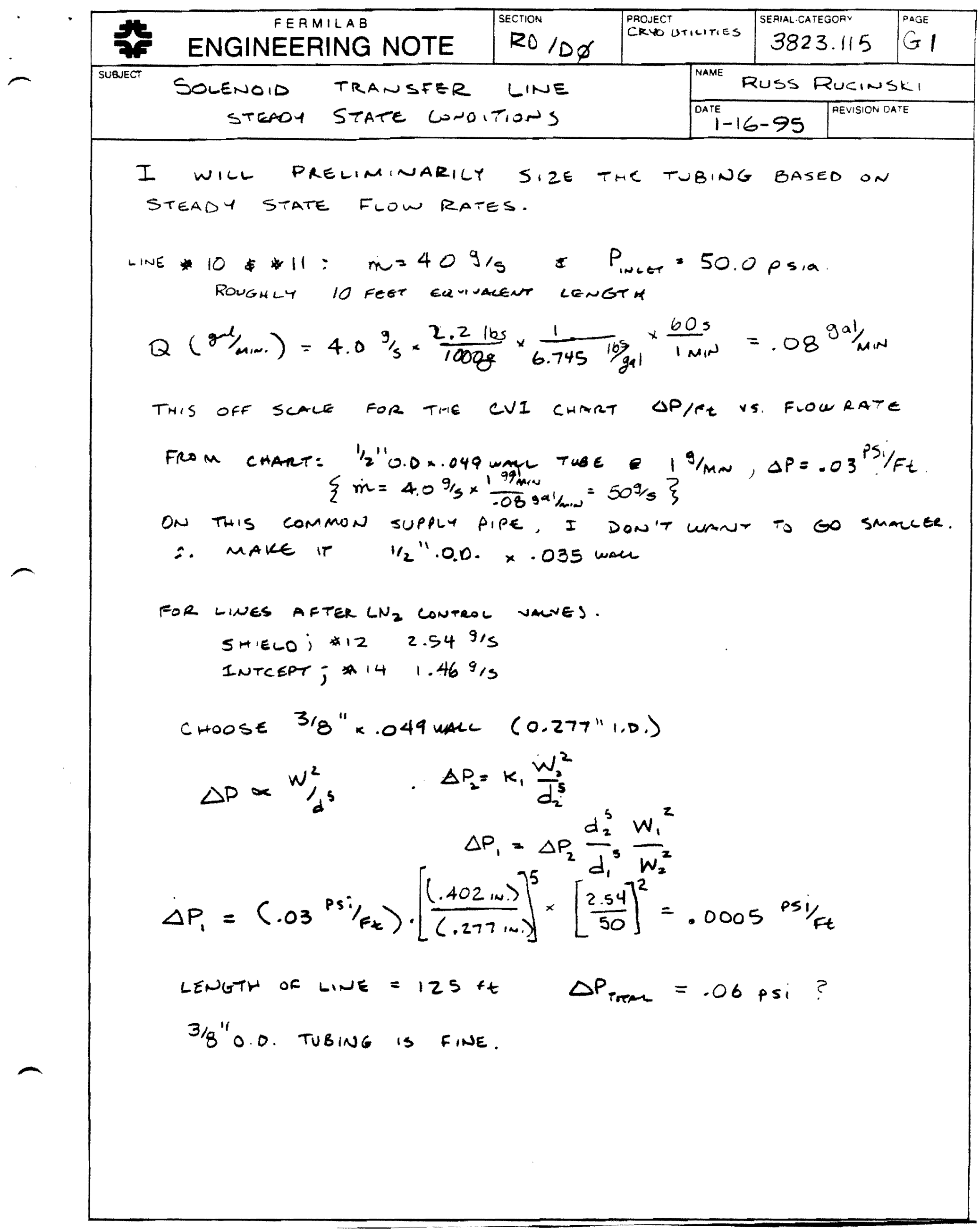


The following curves may be helpful in determining the proper line size for your transfer requirement. These charts represent loss of pressure due to fluid flow through a pipe. Total pressure drop also includes additional loss of pressure (head) due to any increase in elevation
Please notice that a smaller diameter rigid pipe line can accommodate the same flow capacity as a larger diameter flex line. This is signficant when considering heat-leak (liquid loss) and system purchase cost.

\section{LIOUID NITROGEN}

Pressure Drop Due to Fluid Flow Rigid Piping __ Flexible Piping - _ _ _...-
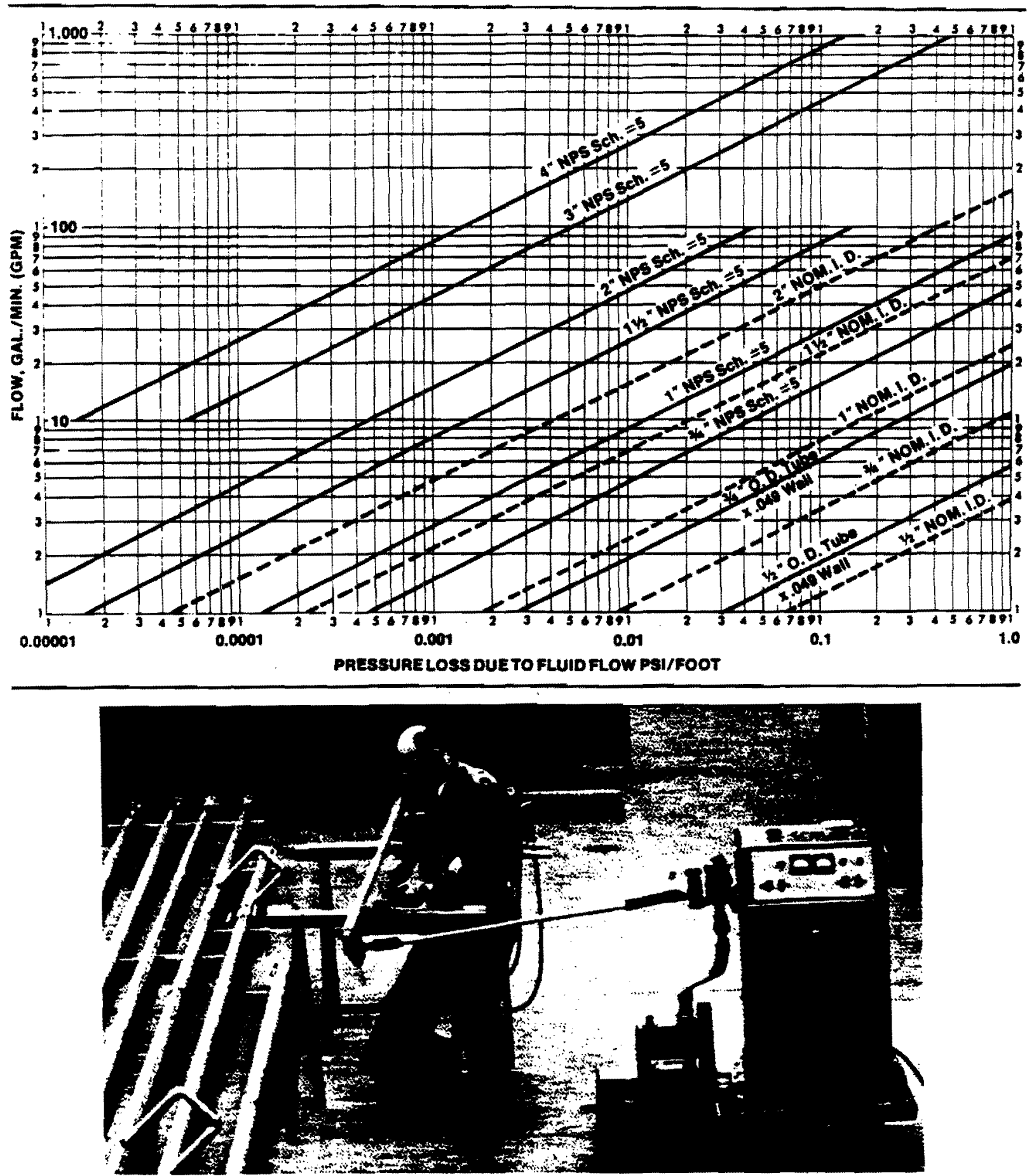

Each piping section undergoes helium mass spectrometer testing $\left(1 \times 10^{-} \mathrm{cc} / \mathrm{sec}\right.$ sensitivity) followed by a seven day vacuum retention test to ensure the vacuum integrity prior to shipment. 


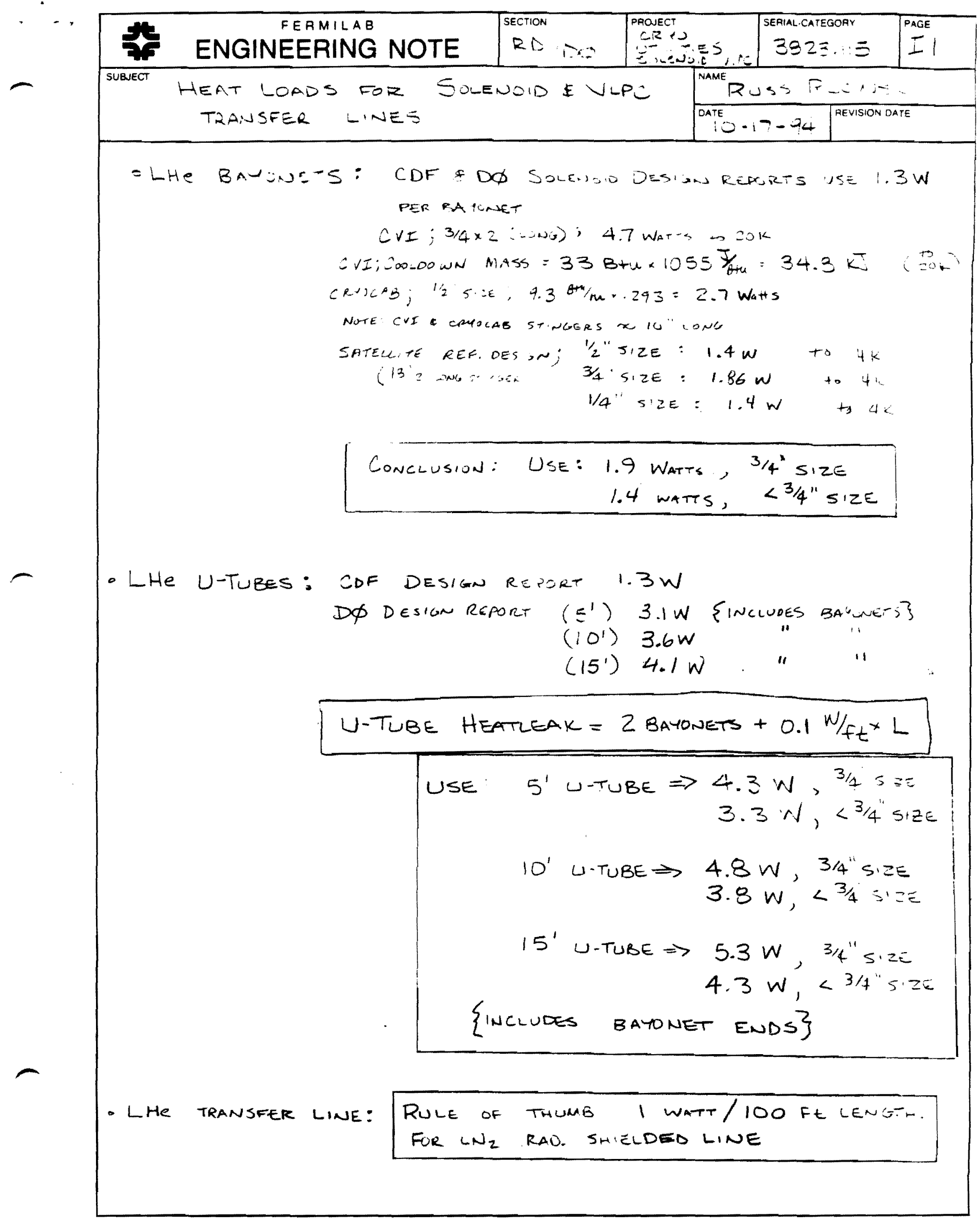




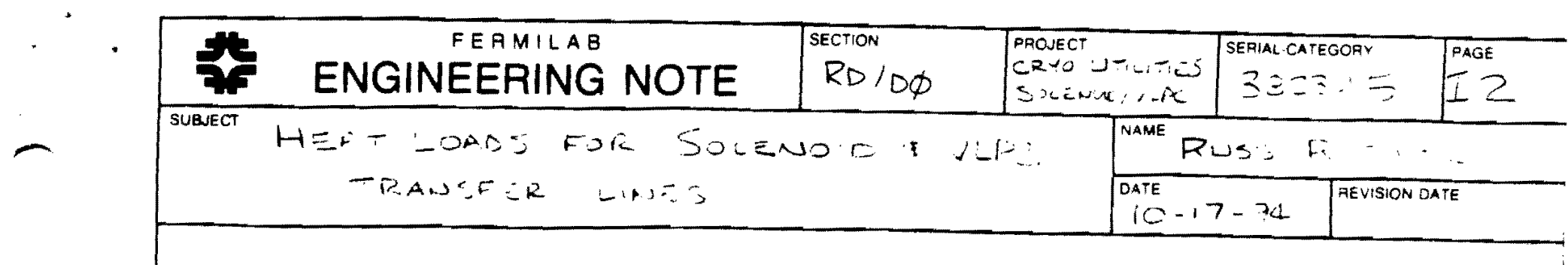

- Lhe control valve: cof des... k..... T. Tha...

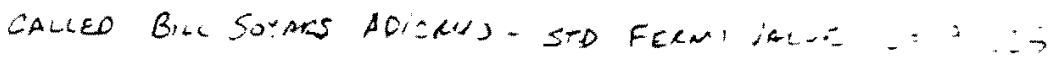

$$
\begin{aligned}
& Q=0.60 \mathrm{~N} \text { to } 4 \mathrm{k}, \mathrm{.} \\
& \text { USE } 0.66 \mathrm{WAT}
\end{aligned}
$$

- trapped volume reliefs:

Assume 1/8" O.D. $\times 0.028$ "NALl tubing. STh. STL.

ASSUME 12 "OF LENGTH BEFORE TJBINE is SUNK $\rightarrow$ LN SHIOD

$$
\begin{aligned}
& Q=\frac{K_{m} A\left(T_{L}-T_{c}\right)}{L}=\left(\frac{A}{L}\right) \cdot\left\{\int_{4 k}^{77} K_{t} d T-\int_{4 k}^{4 c} K_{t} d T\right\} \\
& =\frac{\left(.00853 \mathrm{w}^{2}\right)}{12 \mathrm{~N}} \cdot\{349 \mathrm{w} / \mathrm{m}\} \times .0254 \mathrm{~m} / \mathrm{iN} \text {. } \\
& =.006 \mathrm{~W} \text { Ref. BMaron cemogentic } \\
& \text { INSTRUMENTATION POINTS } \\
& \text { \& VUSE } 0.01 \mathrm{~W} \\
& \text { trapPED VOL.RELIEFS/ PER INSTANi: }
\end{aligned}
$$




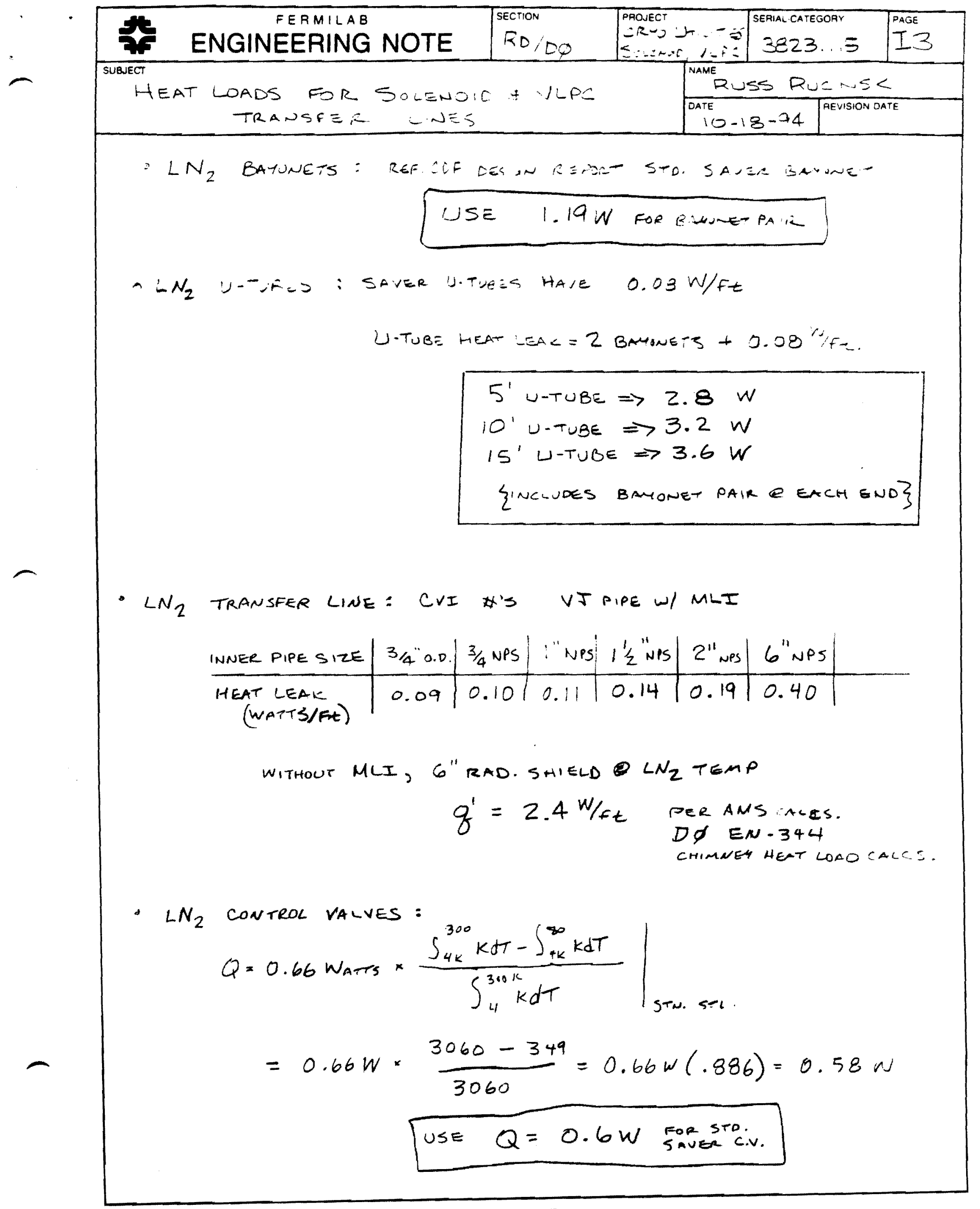




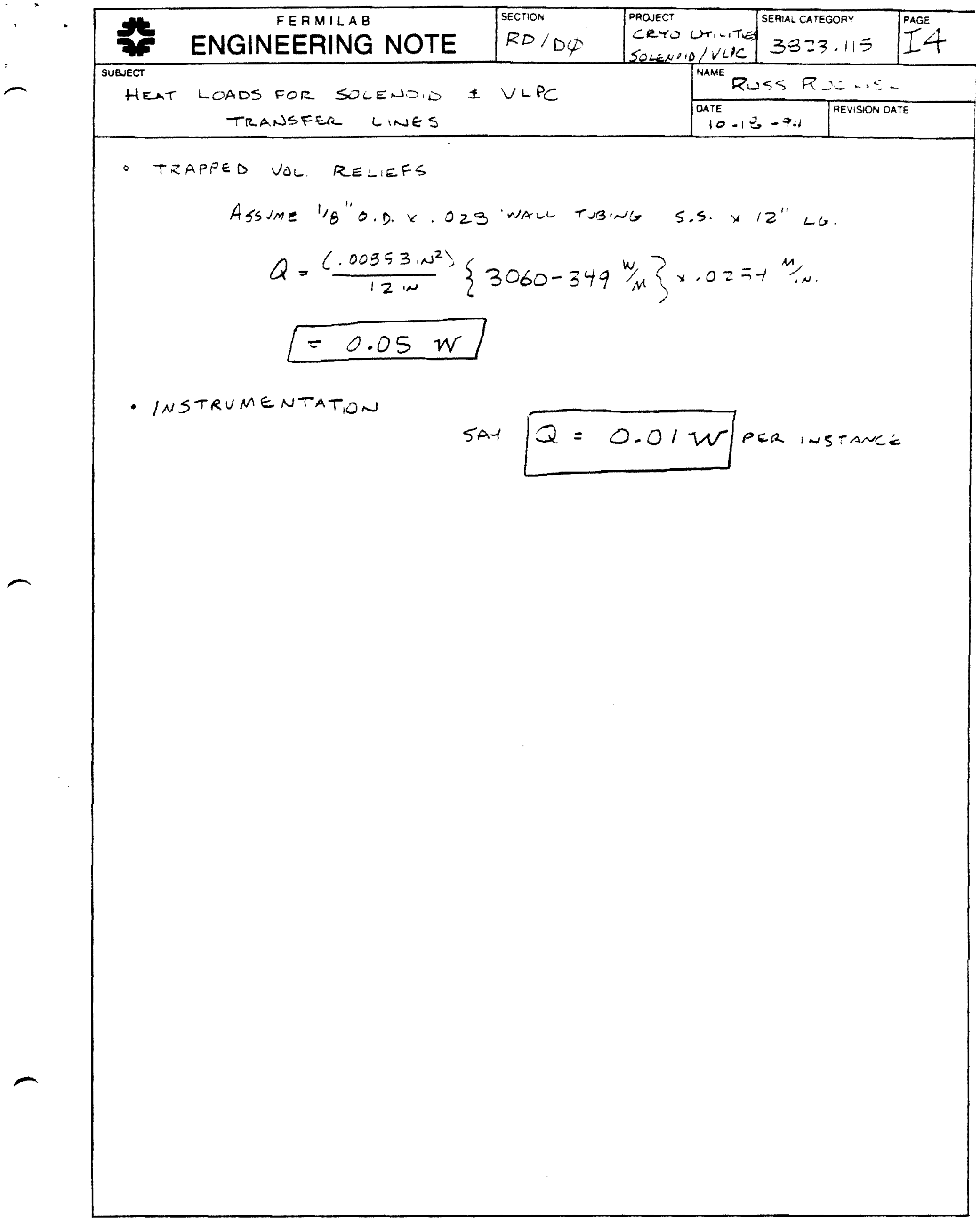




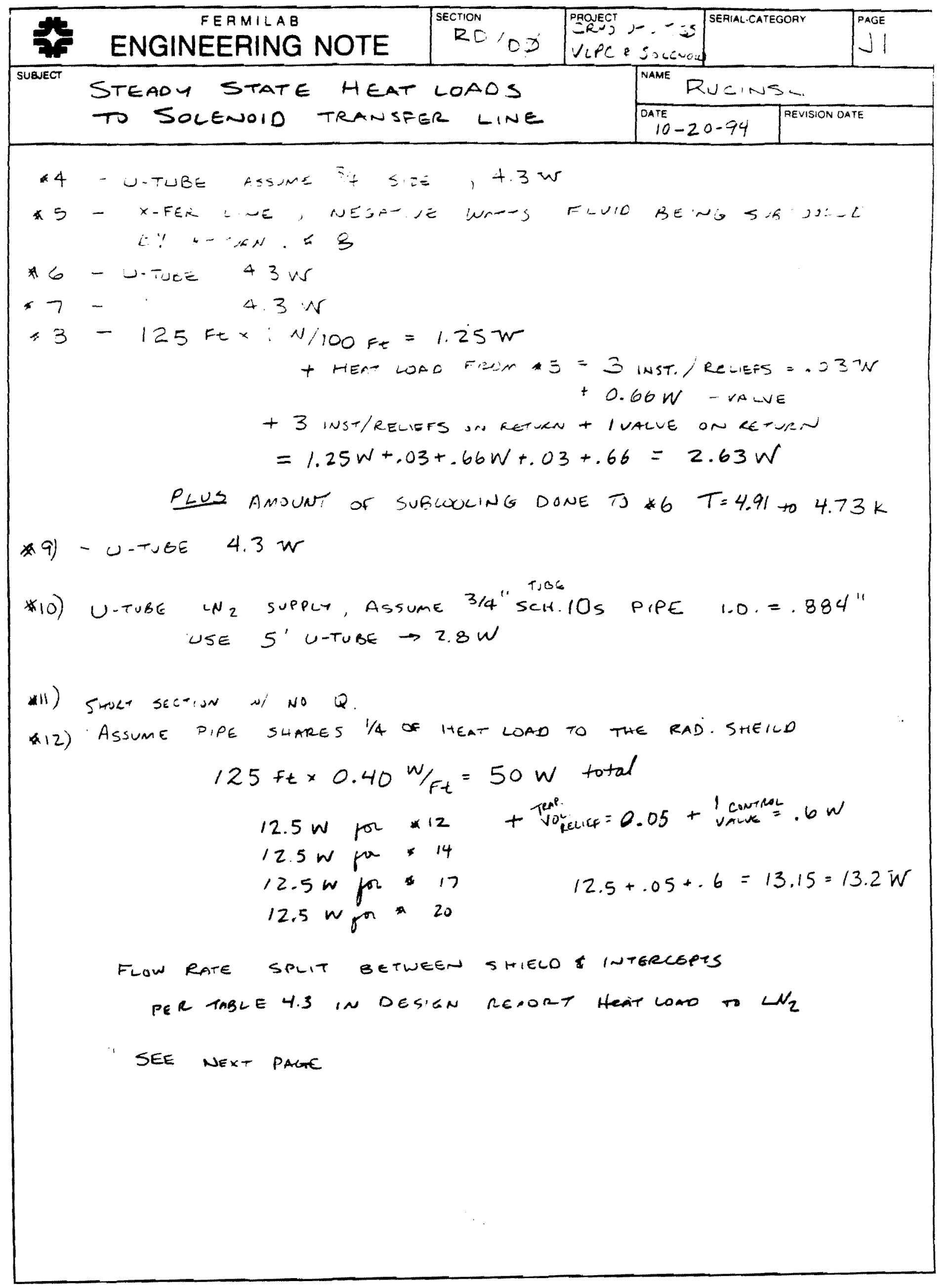




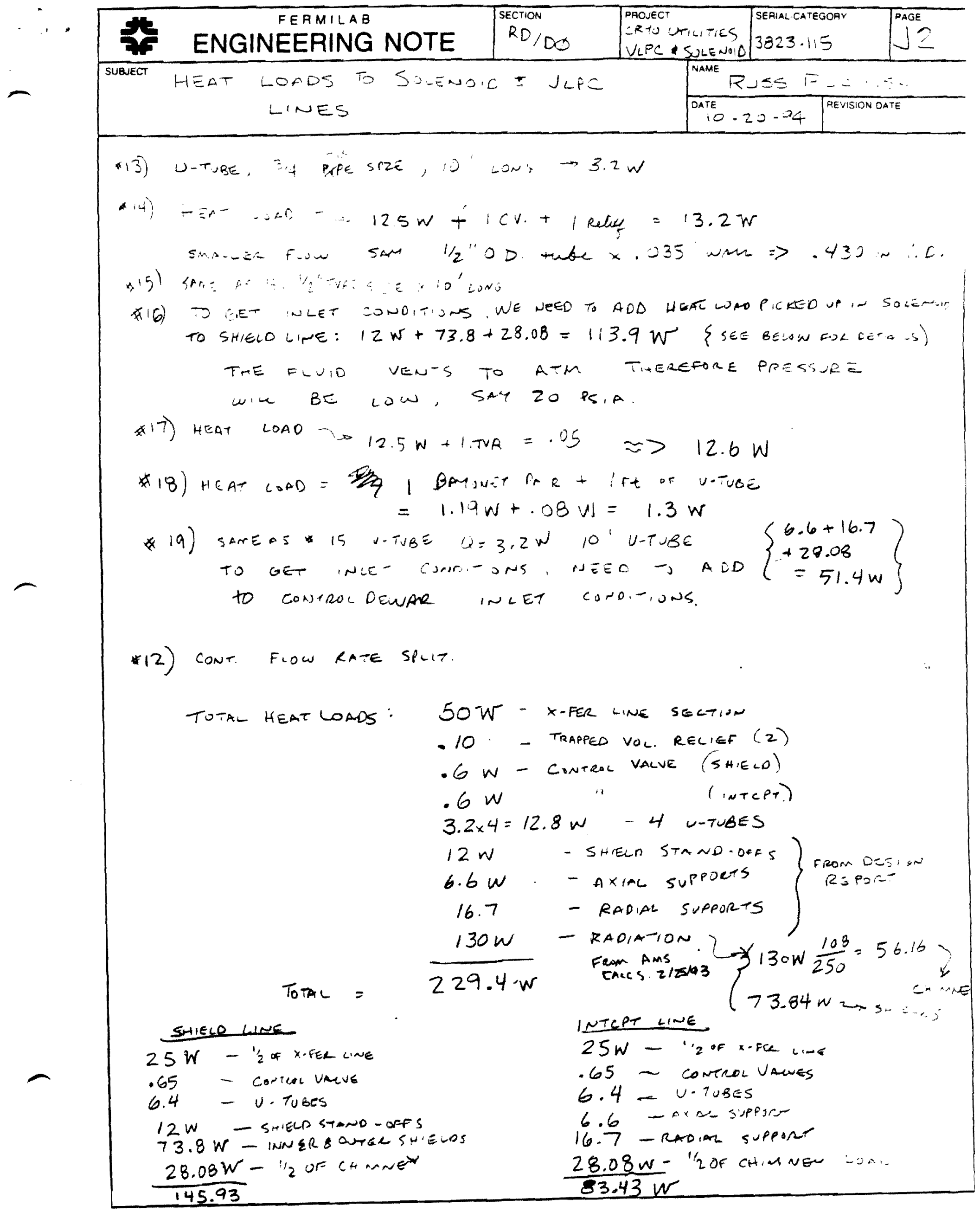




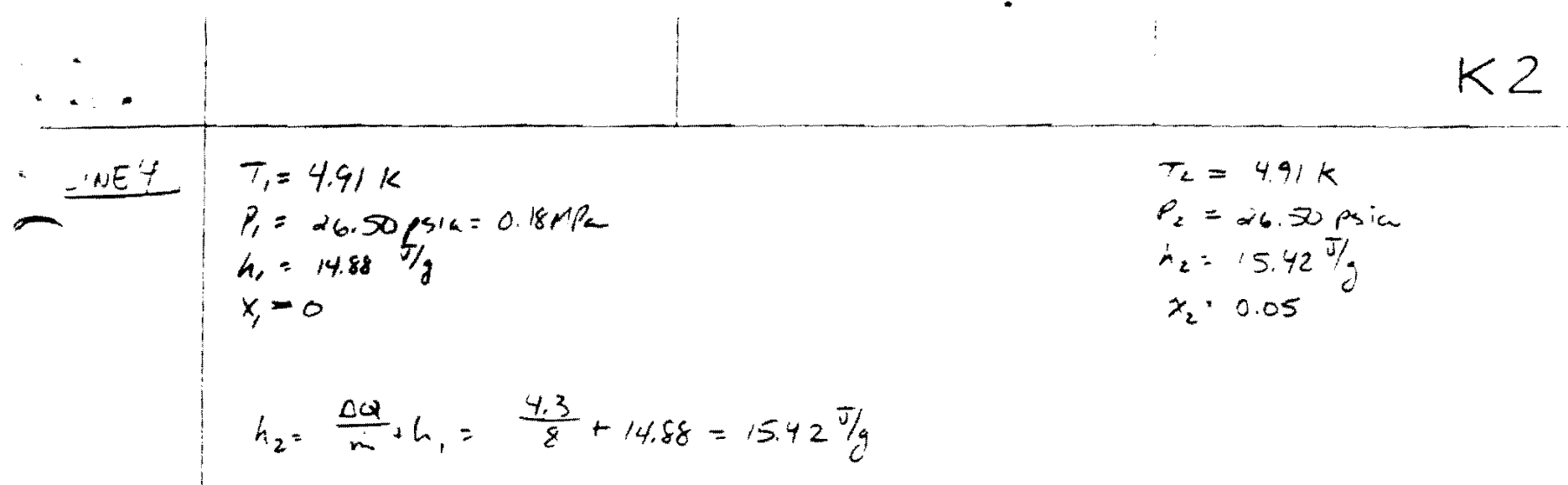

Assume $P_{2}=P_{1}: \quad x_{2}=0.05$

$\Delta P f$ Calculation:

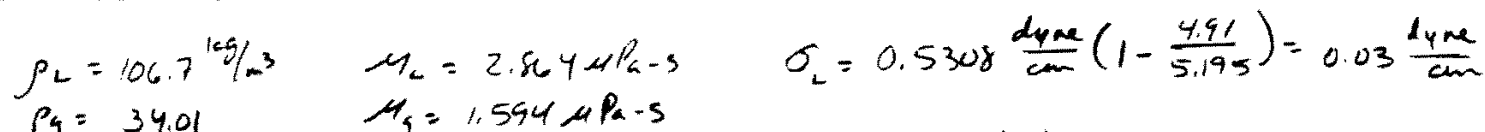

$$
\begin{aligned}
& =3\left(10^{-5}\right) \mathrm{M} / \mathrm{m}
\end{aligned}
$$

$$
\Rightarrow \Delta P_{f}=0.006 p s i g
$$

$\Delta P_{r}$ Calculation:

$\left(k=30 f_{r}\right.$ for $90^{\circ}$ elbows $)$

$$
\begin{aligned}
K=X \frac{L}{D}=307 \\
L=30 D=30\left(\frac{0.62}{12}\right)=1.55 \mathrm{ft}
\end{aligned}
$$

Fran table A-24 $\rightarrow f_{T}=0.0155$

$$
\begin{aligned}
& \Delta P_{T}=\Delta P_{f}\left(\frac{5 f t+2 L}{5+t}\right)=\Delta P_{f}\left(\frac{8.1}{5}\right)=0.01 \text { pig } \\
& P_{2}=26.49 \text { psia }
\end{aligned}
$$


LINEY

$\therefore$ St Input Name Qutput Unit Comment

Lockhart Martinelli correlation

for two phase pressure drop

through an adiabatic horizontal pipe

\begin{tabular}{|c|c|c|c|c|}
\hline & valid & 'accurate & & model validity \\
\hline & Elow & 'turbulen & & flow type \\
\hline .000005 & epsilon & .01620391 & $\mathrm{ft}$ & $\begin{array}{l}\text { friction factor ; Must enter a guess } \\
\text { pipe roughness }\end{array}$ \\
\hline .62 & D & & in & $\begin{array}{l}\text { plpe roughness } \\
\text { Pipe inside diameter }\end{array}$ \\
\hline 5 & $\mathrm{~L}$ & & ft & Length of pipe \\
\hline & $A$ & .00019478 & $m^{\wedge} 2$ & cross sectional area \\
\hline 8 & mdot & & g/s & Total mass flow rate \\
\hline 106.7 & rhoL & & $\mathrm{kg} / \mathrm{m}^{\wedge} 3$ & Liquid density \\
\hline 34.01 & rhog & & $\mathrm{kg} / \mathrm{m}^{\wedge} 3$ & density of the gas \\
\hline 2.864 & muL & & $\mu \mathrm{Pa}-\mathrm{s}$ & Liquid viscosity \\
\hline 1.594 & muG & & $\mu \mathrm{Pa}-\mathbf{s}$ & Gas viscosity \\
\hline .00003 & sigmaL & & $\mathrm{N} / \mathrm{m}$ & $\begin{array}{l}\text { Surface tension of liquid } \\
\text { quality }=\mathrm{mdotg} / \mathrm{mdot}\end{array}$ \\
\hline & $\begin{array}{l}x \\
\text { ReL }\end{array}$ & 214500 & & $\begin{array}{l}\text { quality = mdotG } / \text { mdot } \\
\text { Reynold's \# for liquid }\end{array}$ \\
\hline & mdot L & .0076 & $\mathrm{~kg} / \mathrm{s}$ & Mass flow rate for liquid \\
\hline & ReG & 20290 & & Reynold's \# for gas \\
\hline & mdotG & .0004 & $\mathrm{~kg} / \mathrm{s}$ & Mass flow rate for gas \\
\hline & dpdtu & 7.3408407 & $\mathrm{~Pa} / \mathrm{m}$ & $\Delta \mathrm{P} / \Delta \mathrm{L}$ for the liquid \\
\hline & dpdLTp & 25.168498 & $\mathrm{~Pa} / \mathrm{m}$ & $\Delta \mathrm{P} / \Delta \mathrm{L}$ for the two phase \\
\hline & deltap & .00556327 & psig & Total pressure drop for the pipe \\
\hline & phil & 1.8516366 & & Lockhart-Martinelli parameter \\
\hline & & 8.2850398 & & Lockhart-Martinelli parameter \\
\hline .25 & $\mathbf{m}$ & & & L-M constant; Look up in Table 7.19 \\
\hline .2 & $\mathbf{n}$ & & & L-M constant; Look up in Table 7.19 \\
\hline .316 & CG & & & L-M constant; Look up in Table 7.19 \\
\hline .184 & CL & & & L-M constant; Look up in Table 7.19 \\
\hline 20 & $\mathrm{C}$ & & & L-M constant; Look up in Table 7.19 \\
\hline & lambda & 1.7407229 & & Baker diagram dimensionless parameter \\
\hline 1.2 & rhoair & & $\mathrm{kg} / \mathrm{m}^{\wedge} 3$ & density of air \\
\hline 998 & $\begin{array}{l}\text { rhoH2O } \\
\text { sigh }\end{array}$ & 1534.04 & $\mathrm{~kg} / \mathrm{m}^{\wedge} 3$ & $\begin{array}{l}\text { density of water } \\
\text { Baker diagram dimensionless parameter }\end{array}$ \\
\hline .073 & sigmaH2 & & $\mathbf{N} / \mathbf{m}$ & Surface tension of water \\
\hline 1000 & muH2O & & $\mu \mathrm{Pa}-\mathrm{s}$ & viscosity of water \\
\hline & BakerXa & 50700 & & $x$ - axis value for Baker plot \\
\hline & BakerYa & 870 & $1 \mathrm{bm} / \mathrm{hr}-\mathrm{ft}$ & $Y$ - axis value for Baker plot \\
\hline & $\mathrm{c}$ & 1 & & \\
\hline & $\mathbf{M}$ & 1 & & \\
\hline & RsubL & .54006278 & & Volume fraction of liquid phase \\
\hline & $\begin{array}{l}\text { Ksub } \\
\text { GasVel }\end{array}$ & $\begin{array}{l}.45993 / 22 \\
.1312847\end{array}$ & $\mathrm{~m} / \mathrm{s}$ & Gas velocity \\
\hline & Liqvel & .6771178 & $\mathrm{~m} / \mathrm{s}$ & Liquid velocity \\
\hline
\end{tabular}

